\title{
Isogeometric collocation for large deformation elasticity and frictional contact problems
}

\author{
R. Kruse ${ }^{\mathrm{a}, *}$, N. Nguyen-Thanh ${ }^{\mathrm{a}}$, L. De Lorenzis ${ }^{\mathrm{a}}$, T.J.R. Hughes ${ }^{\mathrm{b}}$ \\ ${ }^{a}$ Institute of Applied Mechanics, Braunschweig University of Technology, Bienroder Weg 87, 38106 Braunschweig, Germany \\ ${ }^{b}$ Institute for Computational Engineering and Sciences, University of Texas at Austin, USA
}

\begin{abstract}
Isogeometric collocation methods have been recently proposed as an alternative to standard Galerkin approaches as they provide a significant reduction in computational cost for higher-order discretizations. In this work, we explore the application of isogeometric collocation to large deformation elasticity and frictional contact problems. We first derive the non-linear governing equations for the elasticity problem with finite deformation kinematics and provide details on their consistent linearization. Some numerical examples demonstrate the performance of collocation in its basic and enhanced versions, differing by the enforcement of Neumann boundary conditions. For problems with strong singularities, enhanced collocation is shown to outperform basic collocation and to lead to a spatial convergence behavior very similar to Galerkin, whereas for weaker or no singularities enhanced and basic collocation may give very similar results. A large deformation contact formulation is subsequently developed and tested in the frictional setting, where collocation confirms the excellent performance already obtained for the frictionless case. Finally, it is shown that the contact formulation in the collocation framework passes the contact patch test to machine precision in a three-dimensional setting with arbitrarily inclined non-matching discretizations, thus outperforming most of the available contact formulations and all those with pointwise enforcement of the contact constraints.
\end{abstract}

Keywords: Isogeometric analysis, Collocation method, Large deformation elasticity, Frictional contact

\section{Introduction}

Isogeometric analysis (IGA) was introduced in [1] with the original objective to tightly integrate computer aided design (CAD) and finite element analysis (FEA). To define geometric entities, CAD makes wide use of non-uniform rational B-splines (NURBS), due to their geometric precision and free-form modeling capability. Using the same functions as basis functions in the FEA setting greatly simplifies mesh generation and results in an exact reproduction of the initial CAD geometry within the realm of analysis. Apart from geometric exactness, IGA has proved in the past ten years to provide remarkable additional advantages compared to conventional ( $C^{0}$ continuous) Lagrangian shape functions, mostly stemming from the higher and tailorable continuity of the basis functions. In addition to NURBS, other functions (either emanating from CAD or not) have been introduced to allow for local refinement, most notably T-Splines [2, 3, 4], polynomial splines over hierarchical T-meshes [5, 6, 7], and hierarchical B-splines/NURBS [8, 9].

A still open issue when using higher-order isogeometric basis functions is how to reduce the computational cost in the generation and assembly of the stiffness matrix for Galerkin methods [10]. Several quadrature strategies have been proposed to exploit the continuity of the basis functions for a reduction in the number of the integration points, see e.g. [11]. An extreme answer to the quest for efficient quadrature schemes recently led to the proposal of the so-called isogeometric collocation (IGA-C) method, where quadrature is eliminated completely [12]. In this method, the strong form of the governing differential equations of the problem at hand is enforced at a set of discrete collocation points, equal in number to the control points. IGA-C has been demonstrated to lead to substantial cost advantages at high orders of the discretization [10].

*Correspondence to: Roland Kruse, Institute of Applied Mechanics, Braunschweig University of Technology, Germany. Email address: r.kruse@tu-braunschweig.de 
Thus far, IGA-C has been evaluated mostly for linear problems, ranging from early works in linear elasticity [13], linear elastostatic and explicit dynamics [14], to beams and plates [15, 16, 17]. The first non-linear application was analyzed in [18] with the study of contact between deformable bodies. Here, the contact problem was formulated in a frictionless setting for large deformation kinematics, however the continuum was treated as linearly elastic, so that only small deformation cases could be tackled. A striking result from this study is that, despite the pointwise evaluation of the contact residual contributions at the surface collocation points, the formulation passes the contact patch test to machine precision. Such a result has never been obtained in the Galerkin setting. For singular problems in linear elasticity it was also shown that the initially proposed collocation scheme, indicated as basic collocation, may suffer from significant loss of accuracy for highly non-uniform meshes. An improved scheme, termed enhanced collocation, was thus proposed. Basic and enhanced collocation differ only in the imposition of Neumann boundary conditions, which in the latter case mimics the integral Galerkin formulation on the domain boundaries. Enhanced collocation has been shown to restore robustness and accuracy for general Neumannn boundary conditions on highly non-uniform meshes in linear elasticity.

Departing from the results summarized above, two main advancements are presented in this paper. First, the continuum formulation is extended to large-deformation elasticity, considering two different hyperelastic constitutive laws. Special attention is given to the consistent linearization of the non-linear problem, as well as to the issue of Neumann boundary conditions. Results obtained from Galerkin, basic collocation and enhanced collocation schemes are compared for uniform and non-uniform meshes. Secondly, the large deformation contact formulation developed in [18] is extended to the frictional setting. Two small-deformation examples are first solved illustrating the comparison between numerical results and analytical solutions. The subsequent example considers two hyperelastic continuum bodies in frictional contact, thus simultaneously demonstrating the developed formulations for continuum and interface. Finally, a contact patch test is presented in a 3D setting with mutually inclined geometries of the contacting bodies, to extend the validity of the previously obtained results. The paper is structured as follows: in Section 2 B-Splines and NURBS basis functions are briefly introduced. Section 3 describes the large deformation elasticity formulation with IGA-C, followed in Section 4 by the illustration of the related examples. Frictional contact is addressed in Section 5 and its examples are reported in Section 6 . The main conclusions are finally summarized in Section 7.

\section{B-Splines and NURBS basis functions}

In this section we review the basics of B-spline and NURBS basis functions, employing standard NURBS terminology. Further details and extensive references can be found in $[19,20]$ among many others.

A B-spline basis of degree $p$ is generated based on a sequence of real numbers called a knot vector

$$
\Xi=\left\{\xi_{1}, \ldots, \xi_{m+p+1}\right\}
$$

where $\xi_{1} \leq \xi_{2} \leq \ldots \leq \xi_{m+p+1}$, each $\xi_{i} \in \mathbb{R}$ is a knot, and $m$ is the associated number of control points, also equal to the number of basis functions. A univariate B-spline basis function $N_{i, p}(\xi)$ is obtained from the so-called Cox-de Boor recursion formula. Starting from $p=0$ where

$$
N_{i, 0}(\xi)= \begin{cases}1 & \xi_{i} \leq \xi<\xi_{i+1} \\ 0 & \text { otherwise }\end{cases}
$$

the basis functions for $p>0$ are obtained from

$$
N_{i, p}(\xi)=\frac{\xi-\xi_{i}}{\xi_{i+p}-\xi_{i}} N_{i, p-1}(\xi)+\frac{\xi_{i+p+1}-\xi}{\xi_{i+p+1}-\xi_{i+1}} N_{i+1, p-1}(\xi)
$$

introducing the convention $0 / 0=0$. If a knot has multiplicity $k$, the smoothness of the B-spline basis is $C^{p-k}$ at that location. In so-called open knot vectors, the first $p+1$ knots and the last $p+1$ terms are equal, so that the basis is interpolatory at the ends.

Once the basis functions are available, a B-spline curve can be constructed as their linear combination 


$$
\mathbf{C}(\xi)=\sum_{i=1}^{m} N_{i, p}(\xi) \mathbf{P}_{i}
$$

where $\mathbf{P}_{i} \in \mathbb{R}^{d_{s}}$ are the so-called control points, and $d_{s}$ is the dimension of the physical space.

Multivariate B-splines are generated through the tensor product of univariate B-splines. If $d_{p}$ denotes the dimension of the parametric space, $d_{p}$ univariate knot vectors are needed:

$$
\Xi^{d}=\left\{\xi_{1}^{d}, \ldots, \xi_{m_{d}+p_{d}+1}^{d}\right\}
$$

where $d=1, \ldots, d_{p}, p_{d}$ is the polynomial degree in the parametric direction $d$, and $m_{d}$ is the associated number of basis functions. Denoting the univariate basis functions in each parametric direction $d$ as $N_{i_{d}, p_{d}}^{d}$, the multivariate basis functions $B_{\mathbf{i}, \mathbf{p}}(\boldsymbol{\xi})$ are obtained from

$$
B_{\mathbf{i}, \mathbf{p}}(\boldsymbol{\xi})=\prod_{d=1}^{d_{p}} N_{i_{d}, p_{d}}^{d}\left(\xi^{d}\right)
$$

where the multi-index $\mathbf{i}=\left\{i_{1}, \ldots, i_{d_{p}}\right\}$ denotes the position in the tensor product structure, $\mathbf{p}=\left\{p_{1}, \ldots, p_{d}\right\}$ indicates the polynomial degree, and $\xi=\left\{\xi^{1}, \ldots, \xi^{d_{p}}\right\}$ is the vector of the parametric coordinates in each parametric direction $d$. B-spline surfaces and solids are obtained for $d_{p}=2$ and $d_{p}=3$, respectively, from a linear combination of multivariate B-spline basis functions and control points as follows

$$
\mathbf{S}(\boldsymbol{\xi})=\sum_{\mathbf{i}} B_{\mathbf{i}, \mathbf{p}}(\boldsymbol{\xi}) \mathbf{P}_{\mathbf{i}}
$$

where the summation is extended to all combinations of the multi-index $\mathbf{i}$.

NURBS basis functions are obtained from a projective transformation of their B-spline counterparts in $\mathbb{R}^{d_{s}+1}$. Univariate NURBS basis functions $R_{i, p}(\xi)$ are given by

$$
R_{i, p}(\xi)=\frac{w_{i} N_{i, p}(\xi)}{\sum_{j=1}^{m} w_{j} N_{j, p}(\xi)}
$$

where $N_{i, p}$ are B-spline basis functions and $w_{i}$ are the corresponding weights. Finally, multivariate NURBS basis functions are obtained as

$$
R_{\mathbf{i}, \mathbf{p}}(\boldsymbol{\xi})=\frac{w_{\mathbf{i}} B_{\mathbf{i}, \mathbf{p}}(\boldsymbol{\xi})}{\sum_{\mathbf{j}} w_{\mathbf{j}} B_{\mathbf{j}, \mathbf{p}}(\boldsymbol{\xi})}
$$

and NURBS surfaces and solids result from

$$
\mathbf{S}(\boldsymbol{\xi})=\sum_{\mathbf{i}} R_{\mathbf{i}, \mathbf{p}}(\boldsymbol{\xi}) \mathbf{P}_{\mathbf{i}}
$$

\section{A collocation framework for large deformation elasticity}

As follows, we develop a finite deformation elasticity formulation within the collocation framework. We first outline the governing equations and their linearization, and finally specialize the results for two widely used hyperelastic material models.

\subsection{Governing equations}

This work considers quasi-static hyperelastic problems. In the reference and current configurations of the body $\mathcal{B}$, whose domains are respectively indicated as $\Omega, \omega \subset \mathbb{R}^{d_{s}}$, the coordinates are respectively denoted as $\mathbf{X}$ and $\mathbf{x}$. They are related by the mapping $\mathbf{x}=\boldsymbol{\varphi}(\mathrm{X})$ inducing the displacement field $\mathbf{u}=\mathbf{x}-\mathbf{X}$ and the deformation gradient $\mathbf{F}=\operatorname{Grad} \mathbf{x}$ with the jacobian $J=\operatorname{det} \mathbf{F}$. We also define two geometrical mappings between the parametric space $\hat{\Omega}=[0,1]^{d_{p}}$ and the physical domains $\Omega$ in the reference and $\omega$ in the current configurations, as follows 


$$
\begin{aligned}
& \mathbf{F}^{r e f}(\boldsymbol{\xi})=\sum_{\mathbf{i}} R_{\mathbf{i}, \mathbf{p}}(\boldsymbol{\xi}) \mathbf{X}_{\mathbf{i}} \\
& \mathbf{F}^{\text {cur }}(\boldsymbol{\xi})=\sum_{\mathbf{i}} R_{\mathbf{i}, \mathbf{p}}(\boldsymbol{\xi}) \mathbf{x}_{\mathbf{i}}
\end{aligned}
$$

where $\mathbf{X}_{i}$ and $\mathbf{x}_{i}$ are the coordinates of the NURBS control points respectively in the reference and current configurations, related by $\mathbf{x}_{i}=\mathbf{X}_{i}+\hat{\mathbf{u}}_{i}$, with $\hat{\mathbf{u}}_{i}$ as the control point displacements.

In the reference configuration, the body is subjected to body forces $\mathbf{B}$, to prescribed displacements $\hat{\mathbf{u}}$ on the Dirichlet portion of the boundary $\Gamma_{d}$, and to (possibly zero) prescribed tractions $\hat{\mathbf{T}}$ on the remaining (Neumann) portion $\Gamma_{t}$. We indicate as $\Gamma=\Gamma_{d} \bigcup \Gamma_{t}$ the boundary of the domain in the reference configuration, with $\Gamma_{d} \cap \Gamma_{t}=\emptyset$. Suitable regularity requirements are assumed to hold for $\mathbf{B}, \hat{\mathbf{u}}$, and $\hat{\mathbf{T}}$. The counterparts of the boundaries in the current configuration are denoted as $\gamma_{d}, \gamma_{t}$ and $\gamma$ for the Dirichlet, Neumann and the entire boundary, respectively.

The momentum balance equation in strong form in the reference configuration is given by

$$
\operatorname{Div} \mathbf{P}+\mathbf{B}=\mathbf{0} \quad \text { in } \Omega
$$

complemented by the Dirichlet boundary conditions

$$
\mathbf{u}=\hat{\mathbf{u}} \quad \text { on } \Gamma_{d}
$$

and by the Neumann boundary conditions

$$
\mathbf{P N}-\hat{\mathbf{T}}=\mathbf{0} \quad \text { on } \Gamma_{t}
$$

In the previous equations, $\mathbf{P}$ is the first Piola-Kirchhoff stress tensor, and $\mathbf{N}$ is the outward normal unit vector to the boundary $\Gamma_{t}$.

As in [14], the collocation method is interpreted herein in a variational sense and applied directly in the isogeometric framework. We remark that an alternative approach would be to view collocation directly as the enforcement of the respective equilibrium equations (strong form of the problem) at discrete collocation points, in contrast to enforcement in an average sense in weak-form methods. The finite deformation elasticity problem in variational form, based on the principle of virtual work, reads

$$
\int_{\Omega} \mathbf{P}: \operatorname{Grad} \mathbf{w} d \Omega=\int_{\Omega} \mathbf{B} \cdot \mathbf{w} d \Omega+\int_{\Gamma_{t}} \hat{\mathbf{T}} \cdot \mathbf{w} d \Gamma
$$

for every test function $\mathbf{w} \in\left[H^{1}(\Omega)\right]^{d_{s}}$ satisfying homogeneous Dirichlet boundary conditions, i.e.,

$$
\mathbf{w}=\mathbf{0} \quad \text { on } \Gamma_{d}
$$

Integrating eq. (16) by parts and rearranging terms leads to

$$
\int_{\Omega}[\operatorname{Div} \mathbf{P}+\mathbf{B}] \cdot \mathbf{w} d \Omega-\int_{\Gamma_{\mathrm{t}}}[\mathbf{P N}-\hat{\mathbf{T}}] \cdot \mathbf{w} d \Gamma=0
$$

Using the isoparametric approach, we seek an approximation $\mathbf{u}^{h}$ to the unknown exact solution field $\mathbf{u}$ of the elastostatic problem in the form

$$
\mathbf{u}^{h}=\sum_{a=1}^{n} R_{a} \hat{\mathbf{u}}_{a}
$$

where $R_{a}$ are the NURBS basis functions described in Section 2 and $\hat{\mathbf{u}}_{a}$ are the unknown displacement control variables. Substitution into eq. (18) yields

$$
\int_{\Omega}\left[\operatorname{Div} \mathbf{P}^{h}+\mathbf{B}\right] \cdot \mathbf{w} d \Omega-\int_{\Gamma_{t}}\left[\mathbf{P}^{h} \mathbf{N}-\hat{\mathbf{T}}\right] \cdot \mathbf{w} d \Gamma=0
$$


We now need a suitable choice of the test functions. In the collocation method, the test function $\mathbf{w}$ is selected as the Dirac delta, which can be formally constructed as the limit of a sequence of smooth functions with compact support that converge to a distribution $[12,14]$, satisfying the so-called sifting property, i.e.,

$$
\begin{gathered}
\int_{\Omega} f_{\Omega}(\mathbf{X}) \delta\left(\mathbf{X}-\mathbf{X}_{i}\right) d \Omega=f_{\Omega}\left(\mathbf{X}_{i}\right) \\
\int_{\Gamma} f_{\Gamma}(\mathbf{X}) \delta\left(\mathbf{X}-\mathbf{X}_{i}\right) d \Gamma=f_{\Gamma}\left(\mathbf{X}_{i}\right)
\end{gathered}
$$

for every function $f_{\Omega}$ continuous about the point $\mathbf{X}_{i} \in \Omega$ and for every function $f_{\Gamma}$ continuous about the point $\mathbf{X}_{i} \in \Gamma$. In the following, the Dirac delta will be indicated as a Dirac delta "function" following conventional terminology.

Let us assume that $d_{s}=2, m_{1}$ and $m_{2}$ are the numbers of control points in the two parametric directions and $n=m_{1} m_{2}$ is the total number of control points. Thus $2 n$ scalar equations are needed to determine the unknown control point displacements. In the collocation scheme, we choose $n$ collocation points $\hat{\tau}_{k l}$ in $\hat{\Omega}$, $k=\left\{1, \ldots, m_{1}\right\}, l=\left\{1, \ldots, m_{2}\right\}$ located at the (tensor product) Greville or Demko abscissae (see [12] and references therein) of the knot vectors. We indicate the physical maps of $\hat{\tau}_{k l}$ through $\mathbf{F}^{\text {ref }}$ and $\mathbf{F}^{\text {cur }}$ as $\tau_{k l}^{\text {ref }}$ and $\tau_{k l}^{c u r}$, respectively. For $k=1, m_{1}$ and $l=1, m_{2}, \tau_{k l}^{r e f}$ are located at the boundary $\Gamma$ (see Figure 1). Separate sets of equations are needed for the patch interior and for the boundaries.

In the patch interior $\Omega$, we write $2\left(m_{1}-2\right)\left(m_{2}-2\right)$ scalar equations by choosing as test functions the Dirac delta functions centered at the interior collocation points $\tau_{k l}^{r e f}, k=\left\{2, \ldots, m_{1}-1\right\}, l=\left\{2, \ldots, m_{2}-1\right\}$. The resulting equations read

$$
\left[\operatorname{Div} \mathbf{P}^{h}+\mathbf{B}\right]\left(\tau_{k l}^{r e f}\right)=\mathbf{0} \quad \tau_{k l}^{r e f} \subset \Omega
$$

i.e., they are the collocated strong form of the equations at $\tau_{k l}^{r e f}$. Each $\tau_{k l}^{r e f} \subset \Gamma_{t}$ is associated with a collocation equation that sets the value of the boundary traction. This corresponds to choosing as test functions the Dirac delta functions centered at the collocation points located on the Neumann boundary. Here a distinction is needed between the collocation points located at the edges $\left(k=1, m_{1}\right.$ and $l=2, \ldots, m_{2}-1$, or $l=1, m_{2}$ and $\left.k=2, \ldots, m_{1}-1\right)$, and those located at the corners of the domain $\left(k=1, m_{1}\right.$ and $l=1, m_{2}$ ). For collocation points located on edges within the Neumann boundary, the equations are

$$
\left[\mathbf{P}^{h} \mathbf{N}-\hat{\mathbf{T}}\right]\left(\tau_{k l}^{r e f}\right)=\mathbf{0} \quad \tau_{k l}^{r e f} \subset \text { edge } \subset \Gamma_{t}
$$

i.e., they are the collocated strong form of the Neumann boundary conditions at $\tau_{k l}^{r e f}$. For collocation points located at corners where two Neumann boundaries meet, [14] showed that the appropriate equations are

$$
\left[\mathbf{P}^{h}\left(\mathbf{N}^{\prime}+\mathbf{N}^{\prime \prime}\right)-\left(\hat{\mathbf{T}}^{\prime}+\hat{\mathbf{T}}^{\prime \prime}\right)\right]\left(\tau_{k l}^{r e f}\right)=\mathbf{0} \quad \tau_{k l}^{r e f} \equiv \operatorname{corner} \subset \Gamma_{t}
$$

where $\mathbf{N}^{\prime}$ and $\mathbf{N}^{\prime \prime}$ are the outward unit normals of the edges meeting at the corner, and $\hat{\mathbf{T}}^{\prime}$ and $\hat{\mathbf{T}}^{\prime \prime}$ are the respective imposed tractions.

Following [18], the approach in which collocation equations are written at the patch interior (eq. (23)) and at the Neumann boundary (eq. (24) or (25)) is denoted in the following as the basic collocation (BC) approach. It coincides with the approach proposed in the first investigations on IGA-C methods, see [14]. Within the framework of linear elasticity, [18] showed that, in some cases, the BC approach may lead to an inaccurate imposition of the Neumann boundary conditions. Mimicking integral Galerkin formulations, it was proposed in [18] to enforce Neumann boundary conditions by considering a combination of area and edge terms, as follows

$$
\left[\operatorname{Div} \mathbf{P}^{h}+\mathbf{B}\right]\left(\tau_{k l}^{r e f}\right)-\frac{C^{*}}{h}\left[\mathbf{P}^{h} \mathbf{N}-\hat{\mathbf{T}}\right]\left(\tau_{k l}^{r e f}\right)=\mathbf{0} \quad \tau_{k l}^{r e f} \subset \text { edge } \subset \Gamma_{t}
$$

where $h$ is the physical mesh size in the parametric direction perpendicular to the edge. This size is computed as the distance between the first two collocation points encountered starting from the edge and traveling in the parametric direction perpendicular to the edge. In order for this approach to be applicable, a suitable value for the constant $C^{*}$ in eq. (26) needs to be determined. In [18], $C^{*}$ was calibrated through numerical 
experiments by minimizing the discrepancy of the results with respect to those obtained with the classical Galerkin formulation. An optimal value of $C^{*}=4$ was found from linear elastic examples. The approach in which collocation equations are written at the patch interior (eq. (23)) whereas enhanced collocation equations are written at the Neumann boundary (eq. (26)) was denoted as enhanced collocation (EC). $\Gamma_{d}$.

No equations are needed at the Dirichlet boundary, as we impose a priori that $u_{i}^{h}\left(\tau_{k l}^{r e f}\right)=\hat{u}_{i}\left(\tau_{k l}^{r e f}\right)$ on

Along similar lines we may find the counterparts in the current configuration of the interior equations, which read as follows

$$
\left[\operatorname{div} \boldsymbol{\sigma}^{h}+\mathbf{b}\right]\left(\tau_{k l}^{c u r}\right)=\mathbf{0} \quad \tau_{k l}^{c u r} \subset \omega
$$

and of the Neumann boundary equations, which read

$$
\left[\boldsymbol{\sigma}^{h} \mathbf{n}-\hat{\mathbf{t}}\right]\left(\tau_{k l}^{c u r}\right)=\mathbf{0} \quad \tau_{k l}^{c u r} \subset \text { edge } \subset \gamma_{t}
$$

or

$$
\left[\boldsymbol{\sigma}^{h}\left(\mathbf{n}^{\prime}+\mathbf{n}^{\prime \prime}\right)-\left(\hat{\mathbf{t}}^{\prime}+\hat{\mathbf{t}}^{\prime \prime}\right)\right]\left(\tau_{k l}^{c u r}\right)=\mathbf{0} \quad \tau_{k l}^{c u r} \equiv \operatorname{corner} \subset \gamma_{t}
$$

for $\mathrm{BC}$, and

$$
\left[\operatorname{div} \boldsymbol{\sigma}^{h}+\mathbf{b}\right]\left(\tau_{k l}^{c u r}\right)-\frac{C^{*}}{h}\left[\boldsymbol{\sigma}^{h} \mathbf{n}-\hat{\mathbf{t}}\right]\left(\tau_{k l}^{c u r}\right)=\mathbf{0} \quad \tau_{k l}^{c u r} \subset \text { edge } \subset \gamma_{t}
$$

for EC. Here $\boldsymbol{\sigma}$ is the Cauchy stress tensor, $\mathbf{b}$ and $\hat{\mathbf{t}}$ are respectively the body load per unit current volume and the prescribed traction per unit current area on the Neumann boundary $\gamma_{t}$, whereas $\mathbf{n}$ is the outward normal unit vector to $\gamma_{t}$.

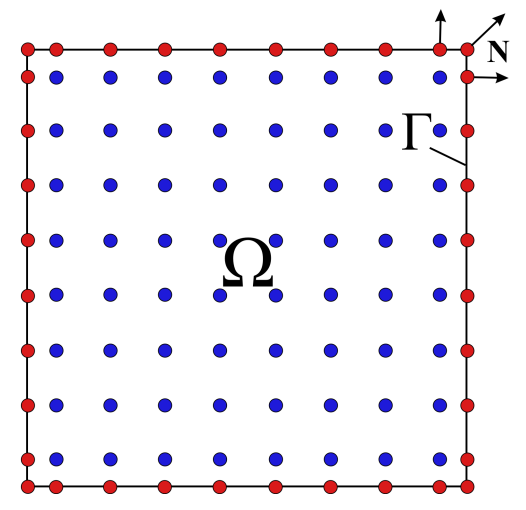

Figure 1: Sketch of the collocation points in the reference configuration. The strong form of the governing equations is enforced on discrete collocation points, here placed according to the Greville abscissae of a uniform knot vector, in the interior $\Omega$ (blue points) and on the boundary $\Gamma$ (red points) of the domain. Vectors $\mathbf{N}$ mark the surface normals.

\subsection{Linearization}

In what follows, we provide some details on the linearization of the governing system of non-linear equations established in Section 3.1.

\subsubsection{Equations in the interior}

Within a Newton-Raphson iterative solution procedure, eq. (23) is rewritten in residual form as

$$
\mathbf{R}=\operatorname{Div} \mathbf{P}+\mathbf{B}
$$

where, for notational simplicity, the superscript $h$ as well as the indication of evaluation at the collocation points $\tau_{k l}^{r e f}$ have been omitted. From finite deformation continuum mechanics (see e.g.[21]) it is known that 
$\mathbf{P}=\mathbf{F S}$, with $\mathbf{S}$ as the second Piola-Kirchhoff stress tensor, so that Div $\mathbf{P}=\mathbf{F D i v} \mathbf{S}+\operatorname{Grad} \mathbf{F}: \mathbf{S}$. Moreover, the term DivS can be developed as

$$
\operatorname{Div} \mathbf{S}=\operatorname{tr}(\operatorname{Grad} \mathbf{S})=\operatorname{tr}\left(\frac{\partial \mathbf{S}}{\partial \mathbf{E}}: \frac{\partial \mathbf{E}}{\partial \mathbf{X}}\right)=\operatorname{tr}(\mathbb{C}: \operatorname{Grad} \mathbf{E})
$$

where $\mathbf{E}$ is the Green-Lagrange strain tensor, and

$$
\mathbb{C}=\frac{\partial \mathbf{S}}{\partial \mathbf{E}}=2 \frac{\partial \mathbf{S}}{\partial \mathbf{C}}
$$

is the fourth-order Lagrangian elasticity tensor, with $\mathbf{C}$ as the right Cauchy-Green deformation tensor. The final expression of the momentum balance equation in residual form is thus

$$
\mathbf{R}=\mathbf{F} \operatorname{tr}(\mathbb{C}: \operatorname{Grad} \mathbf{E})+\operatorname{Grad} \mathbf{F}: \mathbf{S}+\mathbf{B}
$$

or, in indicial notation,

$$
R_{i}=F_{i k} \mathbb{C}_{k j l m} E_{l m, j}+F_{i k, j} S_{k j}+B_{i}
$$

with indices $i, j, k, l, m=1 \ldots d_{s}$. In the above equation, both the stress tensor $\mathbf{S}$ and the elasticity tensor $\mathbb{C}$ are, in general, functions of a deformation measure $(\mathbf{E}$, or $\mathbf{C})$, with the specific function form depending on the constitutive material behavior.

Computation of the consistent tangent stiffness matrix requires linearization of eq. (34), which leads to

$$
\Delta \mathbf{R}=\Delta \mathbf{F} \operatorname{tr}(\mathbb{C}: \operatorname{Grad} \mathbf{E})+\mathbf{F} \Delta \operatorname{tr}(\mathbb{C}: \operatorname{Grad} \mathbf{E})+\operatorname{Grad} \Delta \mathbf{F}: \mathbf{S}+\operatorname{Grad} \mathbf{F}: \Delta \mathbf{S}
$$

with $\Delta$ as the symbol for linearized increment, which commutes with the gradient operator. In the above equation, the following terms can be further developed

$$
\Delta \mathbf{S}=\mathbb{C}: \Delta \mathbf{E}
$$

based on eq. (33),

$$
\Delta \operatorname{tr}(\mathbb{C}: \operatorname{Grad} \mathbf{E})=\operatorname{tr}(\Delta \mathbb{C}: \operatorname{Grad} \mathbf{E}+\mathbb{C}: \operatorname{Grad} \Delta \mathbf{E})
$$

and

$$
\Delta \mathbb{C}=\frac{\partial \mathbb{C}}{\partial \mathbf{E}}: \Delta \mathbf{E}=\mathbb{D}: \Delta \mathbf{E}
$$

where we defined the sixth-order tensor $\mathbb{D}$ as

$$
\mathbb{D}=\frac{\partial \mathbb{C}}{\partial \mathbf{E}}=2 \frac{\partial \mathbb{C}}{\partial \mathbf{C}}
$$

We note that $\mathbb{D}$ is not needed for linearization of the standard Galerkin weak form. With the above manipulations, the linearized residual can be finally expressed as

$$
\Delta \mathbf{R}=\Delta \mathbf{F D i v} \mathbf{S}+\mathbf{F t r}[(\mathbb{D}: \Delta \mathbf{E}): \operatorname{Grad} \mathbf{E}+\mathbb{C}: \operatorname{Grad} \Delta \mathbf{E}]+\operatorname{Grad} \Delta \mathbf{F}: \mathbf{S}+\operatorname{Grad} \mathbf{F}:(\mathbb{C}: \Delta \mathbf{E})
$$

or, in indicial notation, as

$$
\begin{aligned}
\Delta R_{i}= & \Delta F_{i k} S_{k j, j}+F_{i k}\left[\mathbb{D}_{k j l m n o} E_{n o, j} \Delta E_{l m}+\mathbb{C}_{k j l m} \Delta E_{l m, j}\right] \\
& +\Delta F_{i k, j} S_{k j}+F_{i k, j} \mathbb{C}_{k j l m} \Delta E_{l m}+B_{i}
\end{aligned}
$$

While $\mathbb{D}$ can be readily calculated using standard tensor algebra (for an efficient solution see e.g. [22]), a possible alternative consists in approximating it with finite differences

$$
\mathbb{D}_{i j k l m n} \simeq 2 \frac{\Delta \mathbb{C}_{i j k l}}{\Delta C_{m n}}
$$




\begin{tabular}{|c|c|}
\hline No-split model $[23]$ & Split model $[25,26]$ \\
\hline \hline$\psi=\frac{\mu}{2}\left(I_{1}-3\right)-\mu \ln J+\frac{\lambda}{2}(\ln J)^{2}$ & $\psi=\frac{\mu}{2}\left(\bar{I}_{1}-3\right)+\frac{\kappa}{4}\left(J^{2}-1-2 \ln J\right)$ \\
\hline $\mathbf{S}=\mu\left(\mathbf{I}-\mathbf{C}^{-1}\right)+\lambda \ln J \mathbf{C}^{-1}$ & $\mathbf{S}=\mu J^{-\frac{2}{3}}\left(\mathbf{I}-\frac{1}{3} I_{1} \mathbf{C}^{-1}\right)+\frac{\kappa}{2}\left(J^{2}-1\right) \mathbf{C}^{-1}$ \\
\hline $\mathbb{C}=\lambda \mathbf{C}^{-1} \otimes \mathbf{C}^{-1}+2(\mu-\lambda \ln J) \mathbb{I}$ & $\mathbb{C}=\kappa J^{2} \mathbf{C}^{-1} \otimes \mathbf{C}^{-1}-\kappa\left(J^{2}-1\right) \mathbb{I}-$ \\
& $\frac{2}{3} \mu J^{-\frac{2}{3}}\left[\mathbf{C}^{-1} \otimes \mathbf{I}+\mathbf{I} \otimes \mathbf{C}^{-1}-I_{1}\left(\mathbb{I}+\frac{1}{3} \mathbf{C}^{-1} \otimes \mathbf{C}^{-1}\right)\right]$ \\
\hline $\mathbb{D}=-2 \lambda \mathbb{K}+4(\mu-\lambda \ln J) \mathbb{J}$ & $\mathbb{D}=2\left\{\kappa J^{2}\left(\mathbf{C}^{-1} \otimes \mathbf{C}^{-1} \otimes \mathbf{C}^{-1}-\mathbb{K}\right)-\kappa\left(J^{2}-1\right) \mathbb{J}\right.$ \\
& $\left.+\frac{2}{9} \mu J^{-\frac{2}{3}}\left[\mathbb{L}+3 \mathbb{M}-I_{1}\left(\mathbb{K}-3 \mathbb{J}+\frac{1}{3} \mathbf{C}^{-1} \otimes \mathbf{C}^{-1} \otimes \mathbf{C}^{-1}\right)\right]\right\}$ \\
\hline
\end{tabular}

Table 1: Main material-dependent quantities for the two investigated models.

which, however, requires additional evaluations of the elasticity tensor ( 3 in 2D and 6 in 3D). Obviously, neglecting the term containing $\mathbb{D}$ is also possible but would compromise the asymptotically quadratic convergence of the Newton-Raphson scheme, see also Section 4.5.

In case the momentum equation is written in the current configuration as in eq. (27), the corresponding residual

$$
\mathbf{r}=\operatorname{div} \boldsymbol{\sigma}+\mathbf{b}
$$

can most conveniently be rewritten in terms of Lagrangian quantities (considering that $\operatorname{div} \boldsymbol{\sigma}=J^{-1} \operatorname{Div} \mathbf{P}$ and $\mathbf{b}=J^{-1} \mathbf{B}$ ) and linearized in the reference configuration as shown above.

\subsubsection{Equations at the Neumann boundary}

Linearization of the Neumann boundary conditions eq. (24) (for BC) or eq. (26) (for EC) follows along similar lines and is not reported herein. Note that, considering the standard case of deformation-independent applied pressure $\hat{\mathbf{T}}$, it is $\Delta \hat{\mathbf{T}}=\mathbf{0}$. However, for deformation-dependent loading including contact, linearization is needed and is discussed in Section 5 for the case of frictional contact.

Also in this case, Neumann boundary conditions written in the current configuration (eq. (28) for BC or (30) for EC) can most conveniently be rewritten in terms of material quantities, considering that $\boldsymbol{\sigma}=J^{-1} \mathbf{P F}^{T}$ and $\mathbf{n}=\frac{\mathbf{F}^{-T} \mathbf{N}}{\left|\mathbf{F}^{-T} \mathbf{N}\right|}$, and linearized in the reference configuration.

\subsection{Material models}

In this paper, two hyperelastic neo-Hookean material models are adopted, namely, the model proposed in [23], see also [24], and the model in [25, 26] featuring a volumetric-deviatoric split. These are also briefly indicated as "split" and "no-split" models in the following. The main quantities required for the computation of residual and stiffness for the two models are summarized in Table 1. In the Table, $\psi$ is the elastic strain energy density, $\lambda$ and $\mu$ are the Lamé constants, $\kappa$ is the bulk modulus, $I_{1}=\operatorname{tr} \mathbf{C}=\mathbf{C}: \mathbf{I}$ is the first invariant of $\mathbf{C}$ with $\bar{I}_{1}=J^{-2 / 3} I_{1}$ as its distortional component, and $\mathbf{I}$ is the second order unit tensor $\left(I_{i j}=\delta_{i j}\right.$ with $\delta_{i j}$ as the Kronecker delta). Moreover $\mathbb{I}_{i j k l}=\frac{1}{2}\left[C_{i k}^{-1} C_{j l}^{-1}+C_{i l}^{-1} C_{j k}^{-1}\right]$ and the following sixth-order tensors have been defined

$$
\begin{gathered}
\mathbb{J}_{i j k l m n}=-\frac{1}{2}\left(\mathbb{I}_{i k m n} C_{j l}^{-1}+\mathbb{I}_{j l m n} C_{i k}^{-1}+\mathbb{I}_{i l m n} C_{j k}^{-1}+\mathbb{I}_{j k m n} C_{i l}^{-1}\right) \\
\mathbb{K}_{i j k l m n}=C_{i j}^{-1} \mathbb{I}_{k l m n}+C_{k l}^{-1} \mathbb{I}_{i j m n}+C_{m n}^{-1} \mathbb{I}_{i j k l} \\
\mathbb{L}_{i j k l m n}=C_{i j}^{-1} C_{m n}^{-1} \delta_{k l}+C_{k l}^{-1} C_{m n}^{-1} \delta_{i j}+C_{i j}^{-1} C_{k l}^{-1} \delta_{m n} \\
\mathbb{M}_{i j k l m n}=\mathbb{I}_{i j k l} \delta_{m n}+\mathbb{I}_{i j m n} \delta_{k l}+\mathbb{I}_{k l m n} \delta_{i j}
\end{gathered}
$$




\section{Numerical examples of large deformation elasticity}

In this section, four examples are presented to illustrate the performance of IGA-C in its basic and enhanced versions for hyperelasticity problems in plane strain. We consider first an example without singularities, then two examples with singularities using the same geometries as in [18] to enable a direct comparison with the small deformation case, and finally an additional geometry with corner angles other than $90^{\circ}$ (Figure 2 ). Collocation is here always performed at the Greville abscissae, as in [18] the use of Greville and Demko abscissae led to very similar results.

The reported examples have the main objectives to demonstrate the performance of IGA-C in terms of spatial as well as iterative convergence behavior, and to calibrate the constant $C^{*}$ for the EC method. In this respect, it is of interest to evaluate whether the optimal $C^{*}$ for finite deformation elasticity differs from the one calibrated by [18] for the linearized setting. Moreover, in the present non-linear case this value might in principle depend on the deformation level and sign. As in [18], the role of the mesh uniformity is investigated. Finally, as the use of EC in small deformation elasticity was shown to be needed only for singular problems, we investigate the role of the strength of the singularity on the optimal value of $C^{*}$ for small as well as large deformation cases.

In all examples, to assess spatial convergence the reference solution is computed using an "overkill" mesh with uniform knot vectors and 200x200 control points, of interpolation order $p=5$, solved with the Galerkin method. The normalized displacement error in the $L^{2}$-norm is evaluated as follows

$$
e_{L^{2}}^{R e f}=\frac{\left\|\mathbf{u}^{h}-\mathbf{u}^{r e f}\right\|_{L^{2}}}{\left\|\mathbf{u}^{r e f}\right\|_{L^{2}}}
$$

where $\mathbf{u}^{\text {ref }}$ is the reference displacement solution.

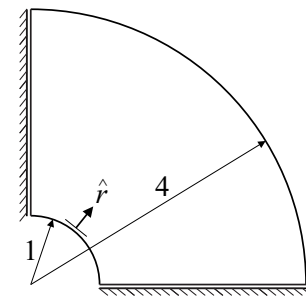

(a)

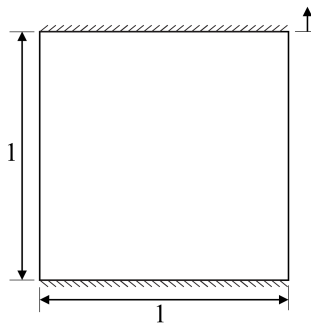

(b)

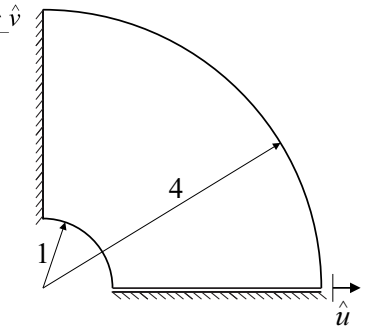

(c)

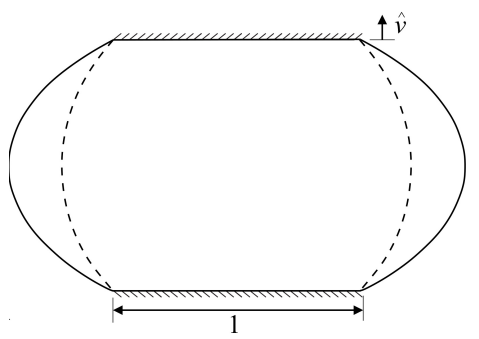

(d)

Figure 2: Examples for large deformation elasticity. (a) Example E1: thick cylinder under inner pressure. (b) Example E2: tension / compression of a square block. (c) Example E3: deformation of a quarter of an annulus by horizontal displacement of its lower edge. (d) Example E4: tension of a barrel-shaped specimen, with moderate ("barrel 1") and strong ("barrel 2") barreling.

\subsection{Example E1: thick cylinder under inner pressure}

\subsubsection{Description}

The first example is a cylinder of inner radius $R_{i}=1$ and outer radius $R_{o}=4$, subjected to inner pressure or, equivalently, to outward radial displacement on the inner radius, see Figure 2a. A quarter of the cylinder is modeled, symmetry boundary conditions are applied on the straight edges, and the imposed displacement is $\hat{r}=0.5$. For this example, the no-split Neo-Hookean model [23] is adopted, with Lamé constants $\mu=1$ and $\lambda=1$. The computations are performed for uniform meshes, for a discretization order $2 \leq p \leq 5$ in both parametric directions.

\subsubsection{Spatial convergence behavior}

The spatial convergence behavior is illustrated in Figure 3. EC computations are reported for $C^{*}=4$, a choice which will be justified later. The observed convergence rates are in excellent agreement with those found in the linearly elastic case [14], equal to $p$ for $p$ even, $p-1$ for $p$ odd with collocation, and $p+1$ with Galerkin. There is no visible difference in accuracy between basic and enhanced collocation, and the Galerkin solution exhibits the best accuracy for all discretization orders and mesh sizes. 


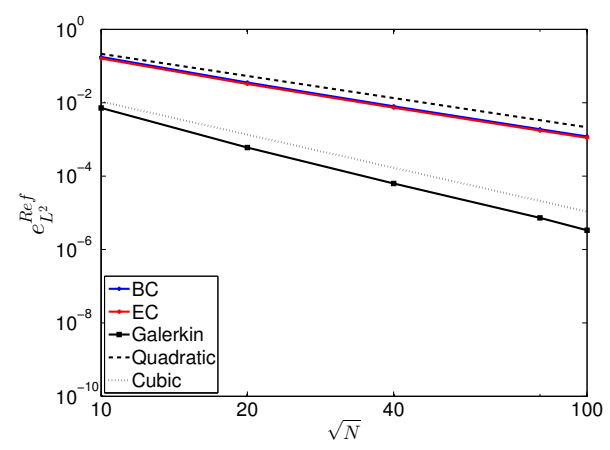

(a)

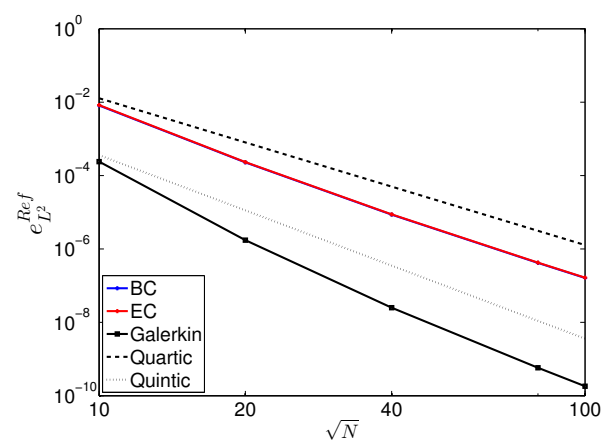

(c)

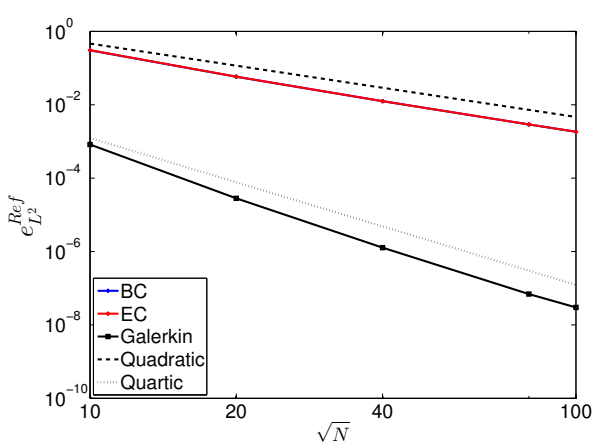

(b)

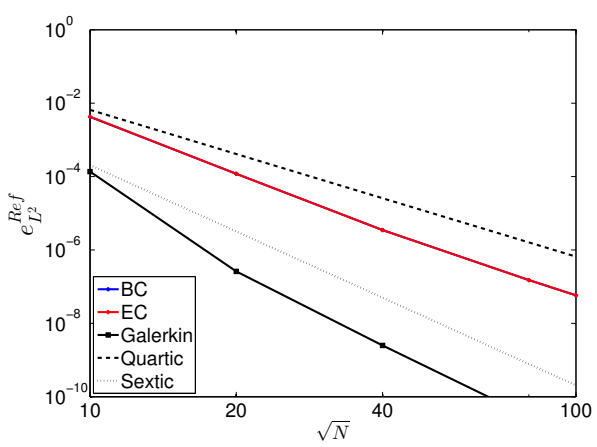

(d)

Figure 3: Example E1: Spatial convergence (EC with $\left.C^{*}=4\right)$. No-split Neo-Hookean model with discretization order 2 (a), 3 (b), 4 (c), 5 (d). Uniform meshes.

\subsection{Example E2: the square block}

\subsubsection{Description}

In the second example we perform a tension / compression test on a square block of side $L=1$, see Figure $2 \mathrm{~b}$. The lower edge has restrained displacements in both directions, whereas the upper edge has restrained displacement in the horizontal direction and a constant imposed displacement in the vertical direction, $\hat{v}$. The vertical sides are both subjected to homogeneous Neumann boundary conditions in both directions. For this example, again the no-split Neo-Hookean model with Lamé constants $\mu=1$ and $\lambda=1$ is adopted.

The displacement $\hat{v}$ on the square block is set to $\hat{v}=0.25,0.50$ and to $\hat{v}=-0.20$ for the tensile and compressive tests, respectively. A lower value of the imposed displacement in compression has been chosen to avoid iterative convergence issues, both with Galerkin and collocation, stemming from severe distortion at the upper corners, including the possibility of self-contact for fine meshes and high discretization orders. The computations are performed for uniform meshes and for non-uniform meshes of aspect ratio (here defined as the ratio between the number of control points in the horizontal to the vertical direction) of 1:4, for a discretization order $2 \leq p \leq 5$ in both parametric directions.

\subsubsection{Spatial convergence behavior}

The spatial convergence behavior is illustrated in Figures 4 and 5 for uniform and non-uniform meshes, respectively. Each figure also illustrates the effect of the imposed deformation level. EC computations are reported again for $C^{*}=4$. The main observations can be summarized as follows:

- for tensile loading cases, BC converges with a similar rate but (with the exception of $p=3$ ) with a lower accuracy with respect to EC and Galerkin. For compressive loading cases and with uniform meshes, $\mathrm{BC}$ fails to converge for $p=2$ and $p=3$, whereas results are similar to those of EC and Galerkin for $p=4$ and $p=5$. For compression loading but non-uniform meshes, no iterative convergence is achieved 
for BC. Thus, unlike in the small deformation setting, in hyperelasticity problems EC may be needed for uniform as well as non-uniform meshes in presence of singularities;

- for $p=2$ and $p=3$, the highest accuracy is exhibited by the Galerkin results, however for the two highest interpolation orders EC converges in all cases with a rate and an accuracy comparable to those of the Galerkin method;

- no significant effect of the deformation level on results is obtained. However, the convergence rate is for all methods consistently lower in the compressive case than in the tensile cases.

\subsubsection{Choice of $C^{*}$ for $E C$}

As mentioned earlier, the EC treatment requires the constant $C^{*}$ to be appropriately selected to achieve maximum accuracy. Since the convergence plots show that the error of the Galerkin method is almost always the smallest, in this section the optimal value of this constant is evaluated through the comparison of EC results with results obtained from the Galerkin method on the same discretization. In particular, the displacement error of each method relative to the Galerkin solution is computed as

$$
e_{L^{2}}^{G a l}=\frac{\left\|\mathbf{u}^{h}-\mathbf{u}_{G}^{h}\right\|_{L^{2}}}{\left\|\mathbf{u}_{G}^{h}\right\|_{L^{2}}}
$$

where $\mathbf{u}_{G}^{h}$ is the Galerkin solution for any given mesh. Results are shown in Figures 6 and 7 . In most cases $e_{L^{2}}^{G a l}$ exhibits a definite minimum, so that the "optimal" value of $C^{*}$ is readily estimated. In most cases, the best agreement between collocation and Galerkin is achieved for $C^{*} \sim 3-4$, with minor increases for compressive loading and high interpolation degree. The range is the same evaluated by [18] for the small deformation case. For $p=3$ under tension loading, except for the coarsest grids, there is no clear minimum in error. On the other hand, in these cases EC provides no improvement in accuracy over BC, as visible from Figures 4 and 5 .

\subsection{Example E3: the quarter of an annulus}

\subsubsection{Description}

The third example has the same geometry as example E1, but different boundary conditions. The left vertical side has restrained displacements in both directions, whereas the lower horizontal side has restrained displacement in the vertical direction and a constant imposed displacement in the horizontal direction, $\hat{u}$. The curved sides are both subjected to homogeneous Neumann boundary conditions in both directions (Figure 2c). For this example, both the no-split [23] and the split Neo-Hookean [25, 26] models introduced in Section 3.3 are adopted, with Lamé constants $\mu=1$ and $\lambda=1$. The displacement $\hat{u}$ is set to 0.25 . The computations are performed for uniform meshes, for a discretization order $2 \leq p \leq 5$ in both parametric directions.

\subsubsection{Spatial convergence behavior and choice of $C^{*}$ for $E C$}

Spatial convergence plots are presented in Figures 8. The main considerations are very similar to those reported for Example E2:

- BC converges with a similar rate but a lower accuracy with respect to EC and Galerkin;

- for $p=2$ and $p=3$, the highest accuracy is exhibited by the Galerkin results, followed by EC and then BC. However, for $p=4$ and $p=5$, EC converges with a rate and an accuracy comparable to those of the Galerkin method.

Figure 9 illustrates the choice of $C^{*}$ for EC. Results are once again similar to those found for Example E2. For $p=2$ and $p=3$, the discrepancy between EC and Galerkin results for each given mesh is almost insensitive to the value of $C^{*}$. For the highest interpolation orders, the behavior changes and a distinct minimum becomes visible, at a slightly higher level than evaluated for Example E2 $\left(C^{*} \sim 5\right)$. However, $C^{*}=4$ is still a good approximation, as shown by the convergence plots in Figure 8 which were evaluated for this value. Finally, the behavior described above appears largely independent of the choice of the investigated material models. 


\subsection{Example E4: the barrel-shaped domain}

\subsubsection{Description}

The fourth example is closely related to example E2, with the lateral sides of the square block now rounded to create barrel-like shapes with corner angles of $136^{\circ}$ and $154^{\circ}$, respectively, see Figure $2 \mathrm{~d}$. Boundary conditions and material model remain unchanged relative to example E2. The larger angle leads to a decrease in the strength of the singularity with respect to Example E2. The objective is to evaluate whether the optimal value of $C^{*}$ is sensitive to the strength of the singularity, both in the small and in the large deformation cases.

The displacement $\hat{v}$ on the barrel-shaped domain is set to $\hat{v}=0.25$. The computations are performed for uniform meshes, for a discretization order $2 \leq p \leq 5$ in both parametric directions.

\subsubsection{Spatial convergence behavior and choice of $C^{*}$ for $E C$}

Spatial convergence plots are to be found in Figure 10. In comparison with the same tensile test on a square block (Figure $4(\mathrm{a}, \mathrm{d}, \mathrm{g}, \mathrm{j}))$ some differences are observed:

- at interpolation orders $p=2,3$ the rate of convergence is reduced compared to the Galerkin solution, especially for the strongly barrel-shaped domain. EC again features a lower error compared to BC;

- at higher interpolation orders, the rate of convergence of the Galerkin solution is recovered and the discrepancy between this solution and $\mathrm{BC}$ results reduced. At the same time, EC with $C^{*}=4$ no longer exhibits spatial convergence.

Figure 11 visualizes the choice of $C^{*}$ for EC. Whereas in example E2 there was a distinct minimum in error at $C^{*} \sim 4$ this is now only the case for the lowest order $p=2$. At orders $p=4,5$ the error decreases continuously with increasing value of $C^{*}$ indicating that $\mathrm{BC}$ would be the best choice in this situation. One maybe be tempted to attribute the increase in optimal $C^{*}$ to the fact that in the barrel-shaped geometries the parametric direction perpendicular to the edge is no longer perpendicular in physical coordinates, hence the chosen parameter $h$ overestimates the 'element thickness'. However, this should only result in a shift of the optimal weight factor towards (slightly) higher values, but not to a better performance of BC over EC.

Since the curves display a nearly asymptotic behavior, EC would still deliver accurate results provided a larger value of $C^{*}$, say $C^{*} \sim 15$, is adopted. Figure 12 illustrates the spatial convergence with this value of $C^{*}$, where we observe a similar and good performance of $\mathrm{EC}$ and $\mathrm{BC}$ in all cases.

For verification purposes example E4 was additionally calculated with small deformation kinematics and the linear elastic material, see Figures 13 and 14. The observed reduced spatial convergence at $p=2,3$ occurs only to a minor extent in this case, and the influence of $C^{*}$ at higher interpolation orders is low. Overall, these results indicate that the choice of $C^{*}$ is only sensitive to the strength of the singularity in large deformation cases, where the optimal $C^{*}$ seems to increase as the strength of the singularity decreases.

In view of the above results, the question arises as to whether $C^{*}=15$ is also sufficiently accurate for the cases where $C^{*}=4$ was previously found to be the optimal choice. To answer this question, spatial convergence plots for all examples were obtained for $C^{*}=15$ and are reported in Appendix A. It appears that the increase in $C^{*}$ leads to limited losses in accuracy for these cases. All in all, the tested examples suggest the use of EC with $C^{*}=15$ as the most suitable choice in large as well as small deformation elasticity problems. However, the optimal selection of $C^{*}$ is an issue which still deserves further investigations.

\subsection{Approximate evaluation of $\mathbb{D}$}

As illustrated in Section 3.2, linearization of the residual at the interior collocation points gives rise to a sixth-order tensor $\mathbb{D}$ which, for the material models considered herein, can be computed according to Table 1 . In order to reduce the costs associated to its computation, an approximate evaluation with finite differences has also been carried out. Results from these two options are compared in Figure 15, which illustrates the iterative convergence history of the Newton-Raphson solver for a representative case of example E2. The direct computation according to Table 1 and the finite difference approximation with $\Delta C_{m n} \sim 10^{-8}$ are shown to lead to identical results. Neglecting the $\mathbb{D}$-related contribution obviously prevents an asymptotically quadratic convergence rate from being achieved. 


\subsection{Large deformation collocation in $3 D$}

To conclude this section we demonstrate the straightforward extension of the presented approach to the $3 \mathrm{D}$ setting with an example similar to the pinched torus in [20]. A hollow torus made of neo-Hookean material is subjected to inner pressure and pinched. Exploiting symmetry, only one eight of the torus is modeled with 50,000 degrees of freedom. The results in terms of deformed shape and stress contour are shown in Figure 16. Even for these large values of deformation no issues regarding stability / iterative convergence were encountered.

\section{Large deformation frictional contact formulation}

As mentioned earlier, the second objective of this contribution is to extend to the frictional regime the IGA-C treatment of contact between multiple patches presented in [18]. This section first introduces the frictional contact problem between deformable bodies, in strong as well as in variational form. Subsequently, we illustrate the treatment of contact constraints within IGA-C based upon the BC and the EC approaches.

\subsection{Elastostatic problem with frictional contact}

Let $\Omega^{(i)} \subset \mathbb{R}^{d_{s}}, i=1,2$, represent the domains occupied in the reference configuration by two elastic bodies $\mathcal{B}^{(i)}$, and $\omega^{(i)}$ be their counterparts in the current configuration. In the reference (current) configuration, each body is subjected to body forces $\mathbf{B}^{(i)}\left(\mathbf{b}^{(i)}\right)$, to prescribed displacements $\hat{\mathbf{u}}^{(i)}$ on the Dirichlet portion of the boundary $\Gamma_{d}^{(i)}\left(\gamma_{d}^{(i)}\right)$, to (possibly zero) prescribed tractions $\hat{\mathbf{T}}^{(i)}\left(\hat{\mathbf{t}}^{(i)}\right)$ on the Neumann portion of the boundary $\Gamma_{t}^{(i)}\left(\gamma_{t}^{(i)}\right)$, and to contact constraints on the remaining portion $\Gamma_{c}^{(i)}\left(\gamma_{c}^{(i)}\right)$. Thus $\Gamma^{(i)}=$ $\Gamma_{d}^{(i)} \cup \Gamma_{t}^{(i)} \cup \Gamma_{c}^{(i)}\left(\gamma^{(i)}=\gamma_{d}^{(i)} \bigcup \gamma_{t}^{(i)} \bigcup \gamma_{c}^{(i)}\right)$ is the reference (current) boundary of the domain of body $\mathcal{B}^{(i)}$ with zero intersections between the Dirichlet, Neumann and contact portions. Suitable regularity requirements are assumed for $\mathbf{B}^{(i)}, \mathbf{b}^{(i)}, \hat{\mathbf{u}}^{(i)}, \hat{\mathbf{T}}^{(i)}$ and $\hat{\mathbf{t}}^{(i)}$. Note that $\Gamma_{c}^{(1)} \neq \Gamma_{c}^{(2)}$ in general, whereas the respective mappings in the current configuration during contact coincide: $\gamma_{c}^{(1)}=\gamma_{c}^{(2)}$.

The finite deformation elasticity problem in strong form for the two bodies in the current configuration consists of the balance equations

$$
\operatorname{div} \boldsymbol{\sigma}^{(i)}+\mathbf{b}^{(i)}=\mathbf{0} \quad \text { in } \omega^{(i)}
$$

complemented by the Dirichlet boundary conditions

$$
\mathbf{u}^{(i)}=\hat{\mathbf{u}}^{(i)} \quad \text { on } \gamma_{d}^{(i)},
$$

by the Neumann boundary conditions

$$
\boldsymbol{\sigma}^{(i)} \mathbf{n}^{(i)}-\hat{\mathbf{t}}^{(i)}=\mathbf{0} \quad \text { on } \gamma_{t}^{(i)}
$$

and by the contact constraints in the normal direction

$$
g_{N} \geq 0 \quad t_{N} \leq 0 \quad g_{N} t_{N}=0 \quad \dot{g}_{N} t_{N}=0
$$

and in the tangential direction

$$
\Phi=\left\|\mathbf{t}_{T}\right\|+\mu t_{N} \leq 0 \quad \dot{\mathbf{g}}_{T}=\dot{\gamma} \frac{\mathbf{t}_{T}}{\left\|\mathbf{t}_{T}\right\|} \quad \gamma \geq 0 \quad \gamma \Phi=0
$$

both valid on $\gamma_{c}^{(s)}$, with $\mu$ as the Coulomb friction coefficient, $\gamma$ as the incremental plastic slip and a superposed dot indicating time derivation. The normal gap is defined as [27, 28]

$$
g_{N}=\left(\mathbf{x}^{(s)}-\overline{\mathbf{x}}^{(m)}\right) \cdot \overline{\mathbf{n}}^{(m)}
$$

In the above, the superscripts $(s)$ and $(m)$ denote the slave and master surfaces, respectively, and the symbol $\left(^{-}\right)$indicates the closest point projection from the slave onto the master surface. Thus $\overline{\mathbf{n}}^{(m)}$ is the normal to the master surface at the (normal) projection point $\overline{\mathbf{x}}^{(m)}$ of a point $\mathbf{x}^{(s)}$ on the slave surface. In the tangential direction, assuming $d_{s}=2$, we can define the tangential gap in incremental form as 


$$
\dot{\mathrm{g}}_{T}=\dot{\bar{\xi}} \overline{\boldsymbol{\tau}}_{1}^{(m)}
$$

assuming that the master surface is parameterized by the convective coordinate $\xi$, that defines the covariant vector $\boldsymbol{\tau}_{1}^{(m)}=\mathbf{x}_{, \xi}^{(m)}$. Using the metric $m_{11}^{(m)}=\boldsymbol{\tau}_{1}^{(m)} \cdot \boldsymbol{\tau}_{1}^{(m)}$ with inverse $m^{11(m)}$, the contravariant vector $\boldsymbol{\tau}^{1(m)}=m^{11(m)} \boldsymbol{\tau}_{1}^{(m)}$ is induced. The above incremental definition is suitable for the time-discretized backward Euler formulation

$$
\left(\mathbf{g}_{T}-\mathbf{g}_{T n}\right) / \Delta t=\left(\bar{\xi}-\bar{\xi}_{n}\right) / \Delta t \overline{\boldsymbol{\tau}}_{1}^{(m)}
$$

Here and in the following, all quantities will refer by default to the current time step $t=t_{n+1}$, whereas the subscript $n$ as in eq. (58) will be used to refer to the previous time step $t_{n}$.

The normal contact traction $t_{N}$ and the tangential contact traction $\mathbf{t}_{T}$ are defined in terms of the contact traction vector in the current configuration $\mathbf{t}=\mathbf{t}^{(m)}=-\mathbf{t}^{(s)}$, which is resolved into its normal and tangential components

$$
\mathbf{t}=\mathbf{t}_{N}+\mathbf{t}_{T}=t_{N} \overline{\mathbf{n}}^{(m)}+t_{T_{1}} \overline{\boldsymbol{\tau}}^{1(m)}=t_{N} \overline{\mathbf{n}}^{(m)}+t_{T}^{1} \overline{\boldsymbol{\tau}}_{1}^{(m)} \quad t_{N}=\mathbf{t} \cdot \overline{\mathbf{n}}^{(m)}
$$

In the formulation adopted herein, each surface is taken alternatively as slave and master according to the so-called two-half-pass algorithm [29]. This differs from the classical contact treatments where one surface is chosen as master and the other one as slave. See Section 5.4 for more details.

\subsection{Variational formulation}

The weak form of the balance equations in Section 5.1 is

$$
G(\mathbf{u}, \mathbf{w})=\sum_{i=1}^{2} G_{i}\left(\mathbf{u}^{(i)}, \mathbf{w}^{(i)}\right)+G_{c}=0
$$

where

$$
G_{i}=\int_{\omega^{(i)}} \boldsymbol{\sigma}^{(i)}: \operatorname{grad} \mathbf{w}^{(i)} d \omega-\int_{\omega^{(i)}} \mathbf{b}^{(i)} \cdot \mathbf{w}^{(i)} d \omega-\int_{\gamma_{t}^{(i)}} \hat{\mathbf{t}}^{(i)} \cdot \mathbf{w}^{(i)} d \gamma
$$

and

$$
G_{c}=-\sum_{i=1}^{2} \int_{\gamma_{c}^{(i)}} \mathbf{t}^{(i)} \cdot \mathbf{w}^{(i)} d \gamma
$$

where $\mathbf{w}^{(i)}$ are the test functions which are assumed to be of sufficient regularity and to satisfy the homogeneous Dirichlet boundary conditions

$$
\mathbf{w}^{(i)}=\mathbf{0} \quad \text { on } \gamma_{d}^{(i)}
$$

Integrating by parts and rearranging terms leads to

$$
\begin{array}{r}
\sum_{i=1}^{2}\left[\int_{\omega^{(i)}}\left[\operatorname{div} \boldsymbol{\sigma}^{(i)}+\mathbf{b}^{(i)}\right] \cdot \mathbf{w}^{(i)} d \omega-\int_{\gamma_{t}^{(i)}}\left[\boldsymbol{\sigma}^{(i)} \mathbf{n}^{(i)}-\hat{\mathbf{t}}^{(i)}\right] \cdot \mathbf{w}^{(i)} d \gamma\right. \\
\left.-\int_{\gamma_{c}^{(i)}}\left[\boldsymbol{\sigma}^{(i)} \mathbf{n}^{(i)}-\mathbf{t}^{(i)}\right] \cdot \mathbf{w}^{(i)} d \gamma\right]=0
\end{array}
$$

The discretized version of eq. (64) is obtained through the use of eq. (19). Once again, the governing equations of the collocation method can be found by taking as test functions the Dirac delta functions centered at the collocation points located on both bodies. The resulting equations are eq. (27) in the interior of both bodies, eqs. (28) or (30) on the Neumann boundaries, plus deformation-dependent Neumann boundary conditions on the contact boundary, which are discussed in Section 5.3. Note that all equations can equivalently be written in the reference configuration, which facilitates their linearization. However, the contact constraints should be enforced in the current configuration due to the use of the two-half-pass algorithm, see Section 5.4 for more details. 


\subsection{Basic and enhanced collocation enforcement of contact constraints}

As visible from eq. (64), using the Dirac delta functions centered at the collocation points located on the contact boundary, the contact constraints can be rewritten as deformation-dependent Neumann boundary conditions and thus straightforwardly enforced with the BC and EC methods presented in Section 3.1. The corresponding equations are obtained by simply substituting the known pressure $\hat{\mathbf{t}}$ at the Neumann boundary with the contact traction, which is a priori unknown and depends on the deformation of the two bodies. Therefore, an active set strategy is needed in order to determine the active collocation points. The computation of the contact traction depends on the solution method chosen for the enforcement of the contact constraints. The simplest option, adopted herein, is the penalty method. The penalty regularized normal contact constraint reads

$$
t_{N}=\varepsilon_{N}\left\langle g_{N}\right\rangle_{-} \quad\left\langle g_{N}\right\rangle_{-}= \begin{cases}g_{N} & \text { if } g_{N} \leq 0 \\ 0 & \text { otherwise }\end{cases}
$$

where $\varepsilon_{N}>0$ is the normal penalty parameter. In the tangential direction, the regularized frictional constraint in the time-discretized setting (using backward Euler integration) takes the form

$$
t_{T}^{1}=t_{T n}^{1}+\varepsilon_{T}\left[\left(\bar{\xi}-\bar{\xi}_{n}\right)-\gamma \frac{t_{T}^{1}}{\left|t_{T}^{1}\right|}\right] \quad \Phi \leq 0 \quad \gamma \geq 0 \quad \Phi \gamma=0
$$

where $\varepsilon_{T}>0$ is the tangential penalty parameter. Application of the classical return mapping algorithm yields the following algorithmic update formula for the frictional traction. The trial state is first computed by assuming that $\gamma=0$

$$
t_{T}^{1 t r}=t_{T n}^{1}+\varepsilon_{T}\left(\bar{\xi}-\bar{\xi}_{n}\right) \quad \Phi^{t r}=\left\|\mathbf{t}_{T}^{t r}\right\|+\mu t_{N}
$$

and the status of stick or slip is then determined based on

$$
t_{T}^{1}= \begin{cases}t_{T}^{1 t r} & \text { if } \Phi^{t r} \leq 0 \\ -\mu t_{N} \frac{t_{T}^{1 t r}}{\left\|t_{T}^{t r}\right\|} & \text { otherwise }\end{cases}
$$

With this method the non-penetration and the stick constraints (for the tangential part) are enforced exactly in the limit as the corresponding penalty parameters approach infinity.

In the BC method, each $\tau_{k l}^{c u r} \subset \gamma_{c}$ is associated with a collocation equation that sets the value of the contact traction. The resulting equations are

$$
\left[\boldsymbol{\sigma}^{h} \mathbf{n}-\mathbf{t}\right]\left(\tau_{k l}^{c u r}\right)=\mathbf{0} \quad \tau_{k l}^{c u r} \subset \text { edge } \subset \gamma_{c}
$$

i.e., they are the collocated strong form of the equilibrium boundary conditions involving the contact pressures at $\tau_{k l}^{c u r}$ and differ from eq. (28) simply by the substitution of the known pressure $\hat{\mathbf{t}}$ with the contact traction t. At collocation points located within the inactive contact boundary, $\mathbf{t}$ vanishes and homogeneous Neumann boundary conditions are automatically recovered. At corners between contact boundaries, equations such as (29) are written with $\mathbf{t}^{\prime}$ and $\mathbf{t}^{\prime \prime}$ in place of $\hat{\mathbf{t}}^{\prime}$ and $\hat{\mathbf{t}}^{\prime \prime}$. Once again, in case of inactive contact one or both of the contact tractions vanish, so that the corresponding contact constraints are automatically replaced by homogeneous Neumann boundary conditions.

Similarly, the contact equations for the EC method can be simply obtained from eqs. (30), by substituting for the pressure $\hat{\mathbf{t}}$ the contact traction $\mathbf{t}$, and read

$$
\left[\operatorname{div} \boldsymbol{\sigma}^{h}+\mathbf{b}\right]\left(\tau_{k l}^{c u r}\right)-\frac{C^{*}}{h}\left[\boldsymbol{\sigma}^{h} \mathbf{n}-\mathbf{t}\right]\left(\tau_{k l}^{c u r}\right)=\mathbf{0} \quad \tau_{k l}^{c u r} \subset \operatorname{edge} \subset \gamma_{c}
$$

\subsection{The two-half-pass algorithm}

In the classical Galerkin setting, contact constraints are usually enforced by considering one of the contacting surfaces as slave and the other one as master. In order to determine the active contact boundary, i.e. the portion of the surfaces where penetration between the bodies takes place, a loop is performed over the discretized slave surface, the values of the gap are evaluated according to eq. (56), and an active contact 
status flag is assigned to the surface regions where $g_{N} \leq 0$. Evaluation of the gap and the corresponding enforcement of the non-penetration constraint may take place at different locations, such as nodes or contact quadrature points, in a local or non-local fashion (see [30] for an overview). Despite the differences between the various approaches, most formulations share two main features: a) they introduce a bias between the slave and the master surfaces, as they prevent the slave body from penetrating into the master body but not vice versa; b) they enforce a priori the local pressure equilibrium between the contacting bodies. The latter is the main reason why these formulations do not fit straightforwardly into the collocation framework [18].

Sauer and De Lorenzis [29] proposed an alternative approach which they called the "two-half-pass formulation", as opposed to the classical procedure termed "full-pass". A similar formulation had been proposed in earlier works by Papadopoulos and co-workers [31]. In this approach, two loops are performed treating each surface alternatively as slave and master. In each loop ("half-pass"), the contact tractions are computed only on the surface currently treated as slave. Therefore, no transfer of tractions to the master side is needed. Local equilibrium at the surfaces is not enforced a priori but has been shown to be recovered with high accuracy. The advantages of the two-half-pass approach are the unbiased treatment of both surfaces as well as an increased degree of robustness observed within the Galerkin setting. The two-half-pass formulation was adopted in [18] to enforce frictionless contact constraints within the collocation framework. It has also been shown [32] that the two-half-pass algorithm (applied in the Galerkin setting) can be straightforwardly extended to the frictional case. Herein, this algorithm is applied to frictional contact within the collocation method.

During each half-pass, one of the potentially contacting surfaces is treated as slave. At each collocation point on this surface, the normal gap and the tangential gap are computed using eqs. (56) and (58), respectively, whereas the normal and tangential contact traction components are computed from eqs. (65) and (68), respectively, and the contact traction vector follows from eq. (59). The latter is then used within eqs. (69) or (70), depending on the specific method used. Note that, as local equilibrium across the contact interface is not enforced a priori in the two-half-pass algorithm, the contact constraints should be enforced in the current configuration in order to ensure matching of the contact domains on the two bodies. The consistent linearization of the contact terms is deferred to Appendix B. Interestingly, linearization of these terms is less cumbersome with collocation than with Galerkin, as the contact equations in residual form contain the expressions of the traction components (and thus of the normal and tangential gaps), whereas the contact residual in the Galerkin method contains the virtual variation of the gaps, thus requiring an additional round of differentiation.

\section{Numerical examples on contact}

In this section, some examples are presented to illustrate the performance of the contact formulation described in the previous section. The first two examples are variants of the Hertz problem with friction, where analytical solutions are available for the kinematically linear case. The subsequent example is the classical ironing contact problem with friction. Finally, a 3D skewed contact patch test is presented.

\subsection{Example C1: the Hertz contact problem with friction on a rigid substrate}

To verify the frictional contact formulation developed in Section 5, we consider contact of a cylinder of radius $R=1$ with a rigid plane in plane strain conditions, see Figure 17a. Figures 17b illustrates the collocation points on the coarsest of the adopted meshes. Note that non-uniform knot vectors are introduced in both parametric directions in order to cluster a sufficient number of collocation points in the vicinity of the contact area. The material of the cylinder is linearly elastic with Young's modulus $E=1.333$ and Poisson's ratio $\nu=0.333$. The penalty parameters are $\varepsilon_{N}=10^{4}, \varepsilon_{T}=10^{3}$ and the friction coefficient is $\mu=0.3$. The cylinder is loaded by a vertical uniformly distributed load $\bar{p}=0.005$ on the upper surface. The analytical solution for this problem is not available in closed form. However, a simplified solution can be obtained by assuming that the normal and tangential contact stresses are uncoupled, which leads to a slight underestimation of the contact pressures [33, 34].

A contour plot of the stress $\sigma_{y y}$ is shown in Figure 18, whereas Figures 19 to 22 compare the normal and tangential components of the contact traction, both normalized with respect to the normal contact traction at the midpoint of the contact area, $t_{N 0}$, as predicted by $\mathrm{BC}$ and by the analytical model for different mesh resolutions and for discretization order (in both parametric directions) ranging from 2 to 5 . The coordinate 
along the contact area is also normalized with respect to the half-size of the contact area, $a$. The BC method provides the values of the contact traction components at the collocation points, and for visualization purposes these are connected linearly. Numerical results are in excellent agreement with analytical predictions. For coarse meshes, the adopted linear interpolation between values across the boundary of the contact area leads to local discrepancies from the analytical solution, whereas the accuracy of the pointwise values is remarkable even for coarse meshes and consistently improves with increasing mesh resolution. Note that in this case there is no need for EC, as the material behavior is linearly elastic and the aspect ratio of the mesh in the vicinity of the contact area is close to the unity.

\subsection{Example C2: the Hertz contact problem with friction between deformable bodies}

The schematic of this problem is shown in Figure 23a, where the values of the geometrical variables are $R=1, b=0.5, h=0.3$. Figures 23b,c visualize the collocation points on the coarsest and the finest of the adopted meshes, respectively. Also in this case, non-uniform knot vectors are used to achieve a local refinement of the contact region albeit within the inherent tensor-product structure of the discretization. The discretization order is $p=2$ in both parametric directions. The material of both bodies is linearly elastic with Young's modulus $E=1$ and Poisson's ratio $\nu=0.3$. The penalty parameters are $\varepsilon_{N}=10^{3}$ and $\varepsilon_{T}=10^{2}$, and the friction coefficient is $\mu=0.2$. The model is loaded with a vertical downward displacement $\hat{v}=0.002$ and an horizontal displacement $\hat{u}=0.00075$. The analytical solution for infinitesimal deformations can be found in [34].

Figure 24 shows the final distribution of the $\sigma_{y y}$ stresses, whereas Figure 25 compares numerical (with BC) and analytical results in terms of normalized components of the contact traction vs. normalized coordinate along the contact area. Once again an excellent agreement is obtained. As in Example C1, BC already gives satisfactory results due to the uniformity of the mesh in the vicinity of the contact region.

\subsection{Example C3: the ironing problem with friction}

A situation with large deformation elasticity is considered next. A half-cylindrical body is pressed onto an elastic slab and then dragged in the tangential direction, see Figure 26a where dimensions and boundary conditions are also indicated. The discretization order is $p=2$ in both parametric directions and the location of the collocation points is shown in Figure 26b. The Neo-Hookean hyper-elastic material behaviour according to the no-split model [23] (see Table 1) is assumed for both bodies, with material parameters $E_{1}=1$ and $\nu_{1}=0.3$ for the slab, and $E_{2}=1000$ and $\nu_{2}=0.3$ for the cylinder. The penalty parameters are $\varepsilon_{N}=10^{3}, \varepsilon_{T}=10^{2}$ and the friction coefficient is $\mu=0.3$. A vertical downward displacement $\hat{v}=0.3$ is applied to the upper face of the cylinder in 20 increments and then held constant while an horizontal displacement $\hat{u}=2.0$ is applied in 130 additional increments.

Figure 27 shows the contour plot of the stress component $\sigma_{y y}$ in the deformed configuration at different time steps for the problem without and with friction $(\mu=0.3)$, whereas Figure 28 reports the reaction forces in the horizontal and vertical directions, computed through numerical integration on the top surface of the half-cylinder, as a function of the pseudo-time increment. The remarkable smoothness of these curves confirms the good performance of the collocation contact algorithm. Also in this case, BC is seen to perform satisfactorily.

\subsection{Example C4: the 3D skewed contact patch test}

The contact patch test proves the capability of a contact formulation to transfer a constant contact pressure across the interface between discretized bodies with non-conforming meshes [35]. This is viewed as a necessary condition for convergence of a contact formulation in the discretized setting. Within the Galerkin framework, it is well known that formulations enforcing the contact constraints in a pointwise manner, such as the node-to-surface (NTS) algorithm, fail the contact patch test, due to local violation of the equilibrium of moments at the contact interfaces [36]. On the other hand, mortar algorithms pass the test, as a result of their non-local enforcement of the contact constraints. However, the test is passed to within the integration error, which is to machine precision for linear elements in the 2D setting, but is larger for higher-order discretizations and always (i.e. including the case of linear discretizations) in the 3D setting.

As follows, we present a (frictionless) contact patch test which extends the setup in [18] to the 3D setting. The cross-section of the upper block has skew sides, so that upper and lower Bézier elements across the contact interface present non-rectangular overlapping areas, see Figure 29. In such a case, mortar methods 
would require the computation of the so called mortar integrals through a quite onerous segmentation of the contact surfaces, which would introduce an integration error in 3D even with the use of bilinear elements. Our aim is to test the performance of the contact formulation within the realm of collocation in this version of the patch test. The two blocks are linearly elastic with Lamé constants $\mu=0.5$ and $\lambda=1.0$, and are pressed onto each other with a uniform pressure $\bar{p}=0.1$ applied in one loading step. Symmetry boundary conditions are enforced on two faces of both blocks. In all points of the lower face of the lower block, zero vertical displacement is imposed. Figure 29b shows that the relative error of the stress $\sigma_{z z}$ (with respect to the exact solution equal to $-\bar{p}$ ), obtained with the $\mathrm{BC}$ approach for a penalty parameter $\varepsilon_{N}=10^{3}$, is at machine precision. This confirms and extends the validity of the result obtained by [18]. As stated therein, the feature of collocation which naturally leads to the satisfaction of the patch test is that the governing equations involve contact pressures instead of nodal concentrated forces. Note that in this example, as the exact solution features uniform deformation and stress states throughout the blocks, the $\mathrm{BC}$ and EC approaches deliver identical results regardless of the aspect ratio of the employed mesh.

\section{Conclusions}

In this paper, we developed and tested the isogeometric collocation method for large deformation kinematics, hyperelastic material behavior and frictional contact. The governing equations for the interior, the Neumann and the contact boundaries were derived and consistently linearized. A thorough study on the computational efficiency of collocation in comparison to Galerkin was outside the scope of this work. However our experience shows that, in the context of large deformation elasticity, collocation offers substantial benefits especially in the 3D case and for higher discretization orders, where a significant portion of the computational time is spent in the matrix-matrix multiplications involved in assembling the element stiffness matrices [10].

Convergence studies for neo-Hookean bodies demonstrated that, for a problem without singularities, basic and enhanced collocation led to identical results and obtained spatial convergence rates equal to the ones previously found for linear elasticity. For problems with singularities, enhanced collocation was shown to achieve a spatial convergence rate and a level of accuracy very similar to Galerkin finite elements, especially for basis functions with order $p>3$. Unlike in the small deformation framework, large deformation kinematics for singular problems may lead to the need for enhanced collocation even for uniform meshes. The weighting coefficient $C^{*}=4$ previously calibrated for the small deformation framework proved suitable also for the investigated hyperelastic cases with the same geometry, where it was also found virtually independent of the deformation level and of the choice of the material model, at least for the cases considered. On shapes with less sharp geometric features (weaker singularity), enhanced collocation was shown to offer no benefit over basic collocation in terms of accuracy or even to have negative effect for the choice $C^{*}=4$. In such cases, the use of a higher value of $C^{*}$ was proved suitable. $C^{*}=15$ ensured iterative and spatial convergence in all examples considered, at the cost of slightly reduced accuracy, and is therefore suggested for hyperelastic as well as linearly elastic problems. Further investigations on the optimal selection of $C^{*}$ are required, including the possibility to introduce a $C^{*}$ value function of the strength of the singularity.

For contact problems between deformable bodies, the excellent performance of the collocated two-half-pass penalty formulation, already known for the frictionless setting, was confirmed in the presence of friction. An excellent agreement was achieved between numerical and analytical contact traction components in problems amenable to a closed-form exact or approximate solution. A large deformation and large sliding frictional ironing example led to remarkably smooth global traction histories in the normal and in the tangential directions. Finally, a three-dimensional skewed contact patch test was shown to be passed to machine precision, which is an unprecedented result for a formulation enforcing the contact constraints in a pointwise manner.

\section{Acknowledgments}

The authors at the TU Braunschweig would like to acknowledge the financial support from the European Research Council Starting Researcher Grant "INTERFACES", Grant agreement No. 279439, and the DFG Priority Program SPP 1748 "'Reliable Simulation Techniques in Solid Mechanics"'. T.J.R. Hughes was supported by grants from the Office of Naval Research (N00014-08-1-0992), the National Science Foundation (CMMI-01101007), and SINTEF (UTA10-000374) with the University of Texas at Austin. 


\section{References}

[1] T.J.R. Hughes, J.A. Cottrell, and Y. Bazilevs. Isogeometric analysis: CAD, finite elements, NURBS, exact geometry and mesh refinement. Computer Methods in Applied Mechanics and Engineering, 194:41354195, 2005.

[2] M. A. Scott, R. N. Simpson, J. A. Evans, S. Lipton, S. P. A. Bordas, T. J. R. Hughes, and T. W. Sederberg. Isogeometric boundary element analysis using unstructured t-splines. Computer Methods in Applied Mechanics and Engineering, 254:197-221, 2013.

[3] Y. Bazilevs, V.M. Calo, J.A. Cottrell, J.A. Evans T.J.R Hughes, S. Lipton, and M.A. Scottand T.W. Sederberg. Isogeometric analysis using T-splines. Computer Methods in Applied Mechanics and Engineering, 199:229-263, 2010.

[4] M. Dörfel, B. Juttler, and B. Simeon. Adaptive isogeometric analysis by local h-refinement with Tsplines. Computer Methods in Applied Mechanics and Engineering, 199:264-275, 2010.

[5] N. Nguyen-Thanh, H. Nguyen-Xuan, S. Bordas, and T. Rabczuk. Isogeometric analysis using polynomial splines over hierarchical T-meshes for two-dimensional elastic solids. Computer Methods in Applied Mechanics and Engineering, 200(21-22):1892-1908, 2011.

[6] N. Nguyen-Thanh, J. Kiendl, H. Nguyen-Xuan, R. Wüchner, K.U. Bletzinger, Y. Bazilevs, and T. Rabczuk. Rotation free isogeometric thin shell analysis using PHT-splines. Computer Methods in Applied Mechanics and Engineering, 200(47-48):3410-3424, 2011.

[7] N. Nguyen-Thanh, J. Muthu, X. Zhuang, and T. Rabczuk. An adaptive three-dimensional RHT-splines formulation in linear elasto-statics and elasto-dynamics. Computational Mechanics, 53:369-385, 2013.

[8] D. Schillinger, L. Dede, M.A. Scott, J.A. Evans, M.J. Borden, E. Rank, and T.J.R. Hughes. An isogeometric design-through-analysis methodology based on adaptive hierarchical refinement of NURBS, immersed boundary methods, and T-spline CAD surfaces. Computer Methods in Applied Mechanics and Engineering, 249-252:116-150, 2012.

[9] A.-V. Vuong, C. Giannelli, B. Jüttler, and B. Simeon. A hierarchical approach to adaptive local refinement in isogeometric analysis. Computer Methods in Applied Mechanics and Engineering, 200:3554-3567, 2012 .

[10] D. Schillinger, J.A. Evans, A. Reali, M.A. Scott, and T.J.R. Hughes. Isogeometric collocation: Cost comparison with Galerkin methods and extension to adaptive hierarchical NURBS discretizations. Computer Methods in Applied Mechanics and Engineering, 267:170-232, 2013.

[11] D. Schillinger, S.J. Hossain, and T.J.R. Hughes. Reduced Bezier element quadrature rules for quadratic and cubic splines in isogeometric analysis. Computer Methods in Applied Mechanics and Engineering, $277: 1-45,2014$.

[12] F. Auricchio, L. Beirao da Veiga, T.J.R. Hughes, A. Reali, and G.Sangalli. Isogeometric collocation methods. Mathematical Models and Methods in Applied Sciences, 20(11):1075-1077, 2010.

[13] W. Sun. B-spline collocation methods for elasticity problems. In P. Minev and Y. Lin, editors, Scientific computing and applications. Nova Science Pub, 2001.

[14] F. Auricchio, L. Beirao da Veiga, T.J.R. Hughes, A. Reali, and G.Sangalli. Isogeometric collocation for elastostatics and explicit dynamics. Computer Methods in Applied Mechanics and Engineering, 14(2):249-252, 2014.

[15] L. Beirao da Veiga, C. Lovadina, and A. Reali. Avoiding shear locking for the Timoshenko beam problem via isogeometric collocation methods. Computer Methods in Applied Mechanics and Engineering, 241:38$51,2012$. 
[16] A. Reali and H. Gomez. An isogeometric collocation approach for Bernoulli-Euler beams and Kirchhoff plates. Computer Methods in Applied Mechanics and Engineering, 284:623-636, 2015.

[17] J. Kiendl, F. Auricchio, L. Beirao da Veiga, C. Lovadina, and A. Reali. Isogeometric collocation methods for the Reissner-Mindlin plate problem. Computer Methods in Applied Mechanics and Engineering, 284:489-507, 2015.

[18] L. De Lorenzis, J.A. Evans, T.J.R. Hughes, and A. Reali. Isogeometric collocation: Neumann boundary conditions and contact. Computer Methods in Applied Mechanics and Engineering, 284:21-54, 2015.

[19] L. Piegl and W. Tiller. The NURBS Book. 2nd edition, Springer-Verlag, Berlin, 1997.

[20] J. Austin Cottrell, Thomas J. R. Hughes, and Y. Bazilevs. Isogeometric Analysis : Toward Integration of CAD and FEA. Wiley, New York, 2009.

[21] G. A. Holzapfel. Nonlinear Solid Mechanics: A Continuum Approach for Engineering. Wiley, 1st edition, 2000 .

[22] P. Chadwick and R.W. Ogden. A theorem of tensor calculus and its application to isotropic elasticity. Archive for Rational Mechanics and Analysis, 44(1):54-68, 1971.

[23] J.C. Simo and K.S. Pister. Remarks on rate constitutive equations for finite deformation. Computer Methods in Applied Mechanics and Engineering, 46:201-215, 1984.

[24] J. Bonet and D. W. Wood. Nonlinear Continuum Mechanics for Finite Element Analysis. Cambridge University Press, 2nd edition, 2008.

[25] J.C. Simo and D.D. Fox. On a stress resultant geometrically exact shell-model 1. formulation and optimal parametrization. Computer Methods in Applied Mechanics and Engineering, 72:267-304, 1989.

[26] J.C. Simo, D.D. Fox, and M.S. Rifai. On a stress resultant geometrically exact shell-model 2. the lineartheory - computational aspects. Computer Methods in Applied Mechanics and Engineering, 73:53-92, 1989 .

[27] P. Wriggers. Computational Contact Mechanics (2nd edn). Springer, Berlin, 2006.

[28] T.A. Laursen. Computational Contact and Impact Mechanics. Springer, Berlin, 2002.

[29] R.A. Sauer and L. De Lorenzis. A computational contact formulation based on surface potentials. Computer Methods in Applied Mechanics and Engineering, 253:369-395, 2013.

[30] L. De Lorenzis, T. J. R. Hughes, and P. Wriggers. Isogeometric contact: a review. In GAMM Mitteilungen, pages 85-123, 2014.

[31] P. Papadopoulos, R. E. Jones, and J. M. Solberg. A novel finite element formulation for frictionless contact problems. International Journal for Numerical Methods in Engineering, 38:2603-2617, 1995.

[32] R.A. Sauer and L. De Lorenzis. An unbiased computational contact formulation for 3d friction. International Journal of Numerical Methods in Engineering, 253, 2014.

[33] D. Nowell, D.A. Hills, and A. Sackfield. Contact of dissimilar elastic cylinders under normal and tangential loading. Journal of the Mechanics and Physics of Solids, 36:59-75, 1988.

[34] D.A. Hills and D. Nowell. Mechanics of Fretting Fatigue. Kluwer Academic Publishers, Dordrecht, 1994.

[35] R. L. Taylor and P. Papadopoulos. On a patch test for contact problems in two dimensions. In P. Wriggers and W. Wanger, editors, Computational Methods in Nonlinear Mechanics. Springer, 1991.

[36] G. Zavarise and L. De Lorenzis. A modified node to segment algorithm passing the contact patch test. International Journal for Numerical Methods in Engineering, 79:379-416, 2009. 


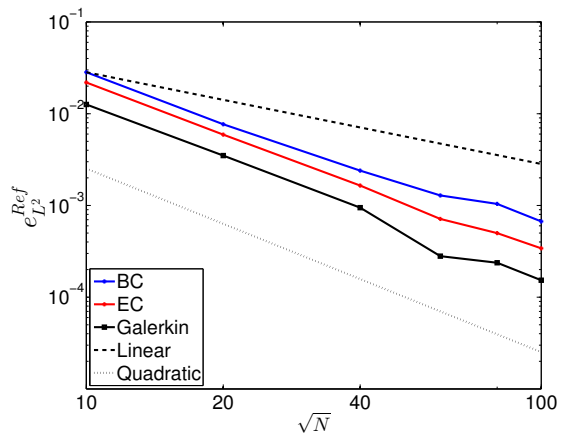

(a)

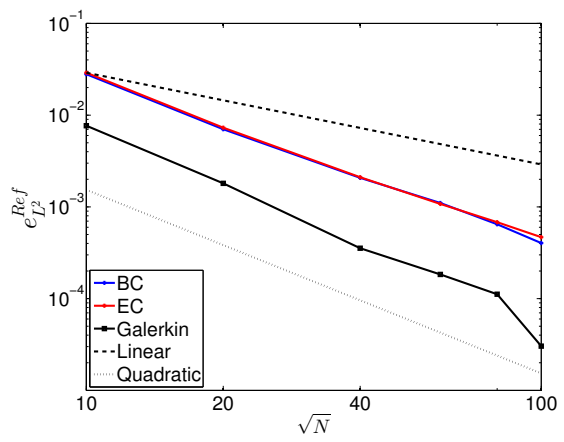

(d)

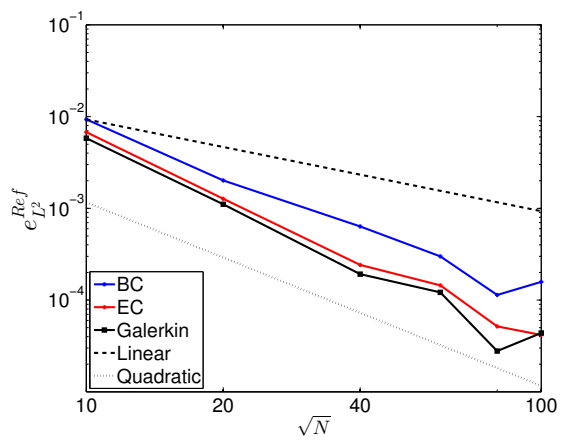

(g)

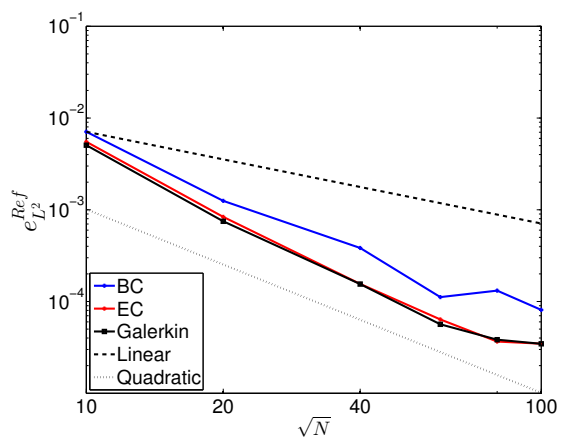

(j)

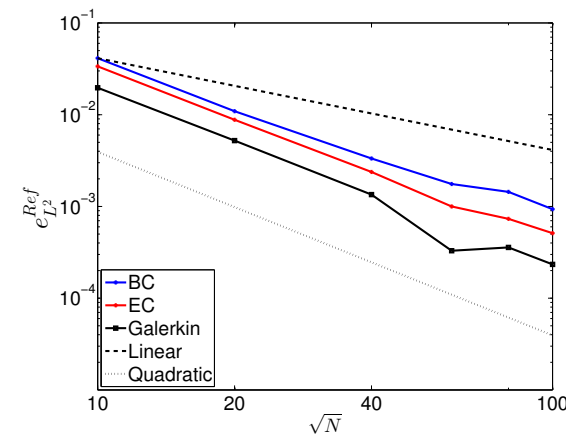

(b)

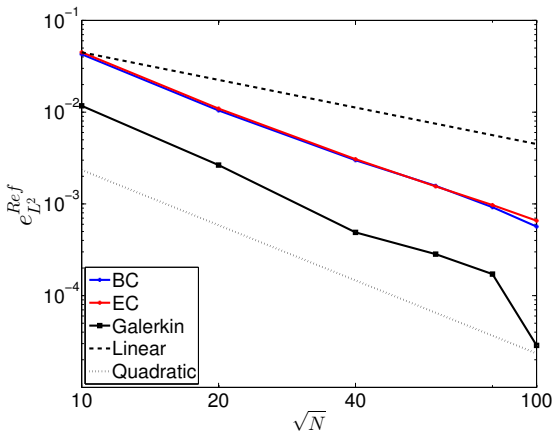

(e)

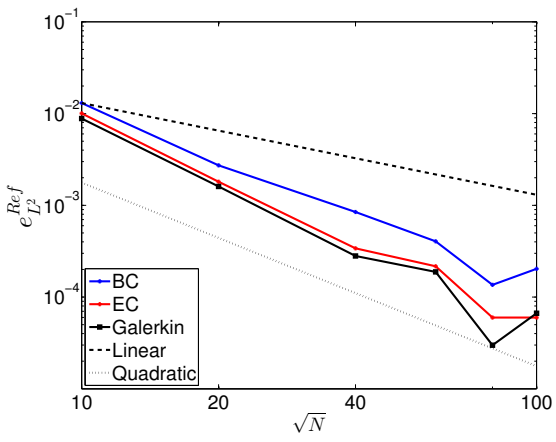

(h)

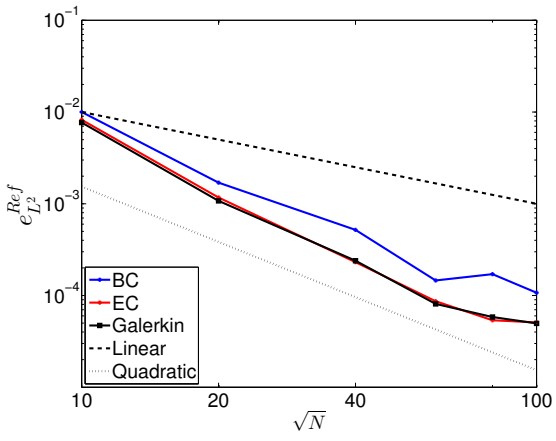

(k)

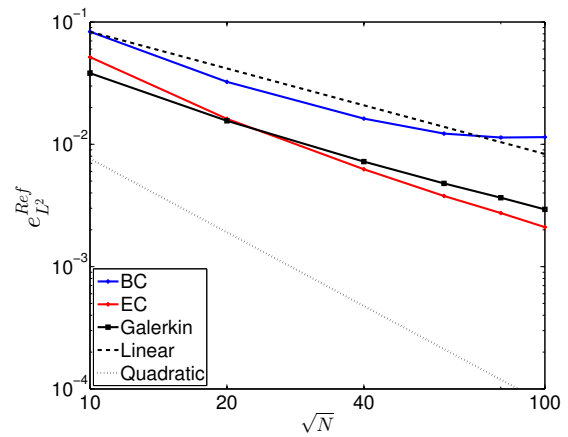

(c)

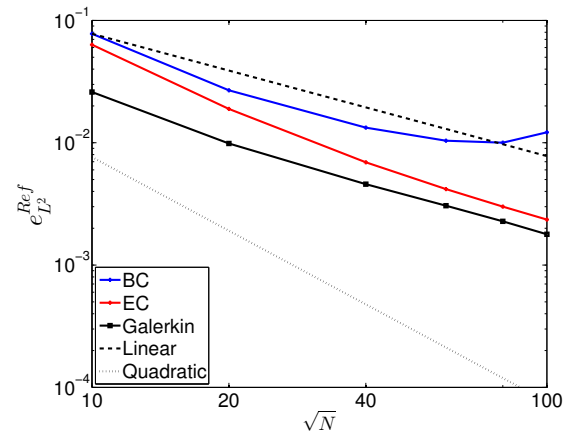

(f)

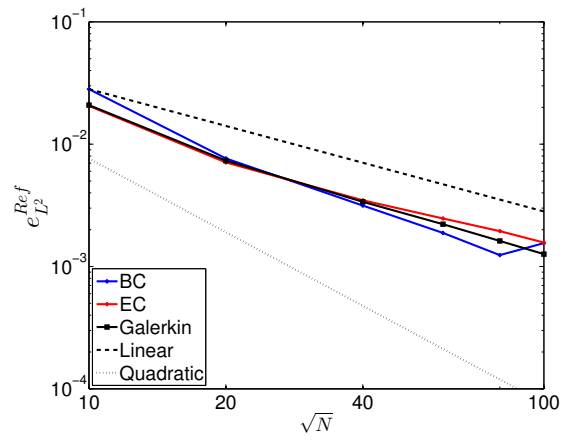

(i)

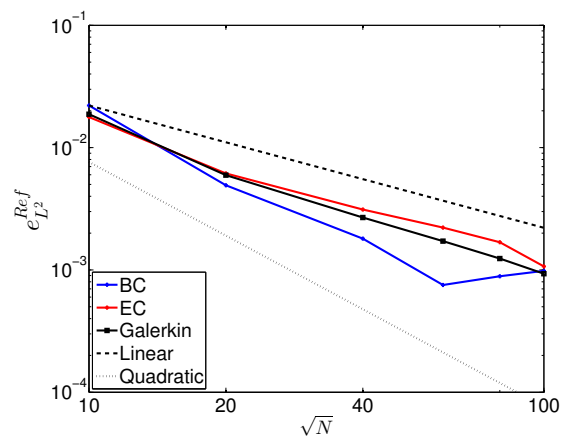

(l)

Figure 4: Example E2: Spatial convergence $\left(\mathrm{EC}\right.$ with $\left.C^{*}=4\right)$ for $(\mathrm{a}, \mathrm{d}, \mathrm{g}, \mathrm{j}) \hat{v}=0.25$, (b,e,h,k) $\hat{v}=0.50$, and $(\mathrm{c}, \mathrm{f}, \mathrm{i}, \mathrm{l}) \hat{v}=-0.20$ with discretization order $2(\mathrm{a}, \mathrm{b}, \mathrm{c}), 3(\mathrm{~d}, \mathrm{e}, \mathrm{f}), 4(\mathrm{~g}, \mathrm{~h}, \mathrm{i}), 5(\mathrm{j}, \mathrm{k}, \mathrm{l})$. No-split Neo-Hookean model and uniform meshes. 


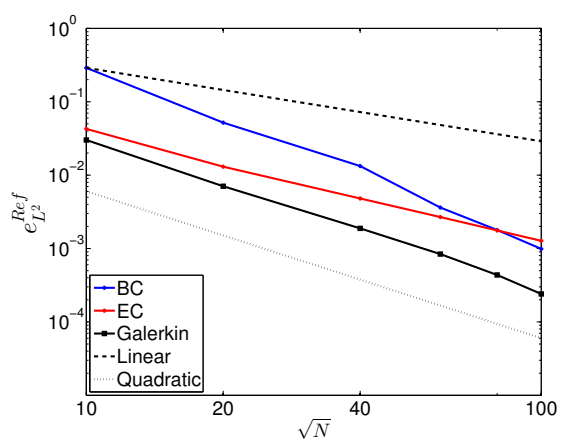

(a)

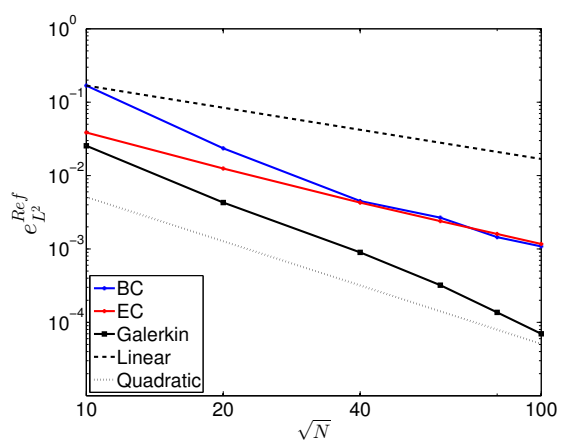

(d)

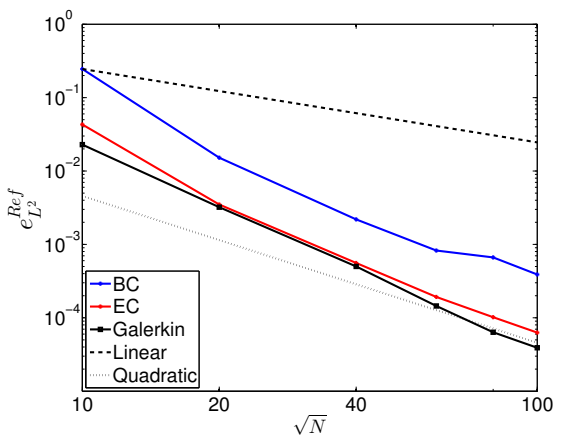

(g)

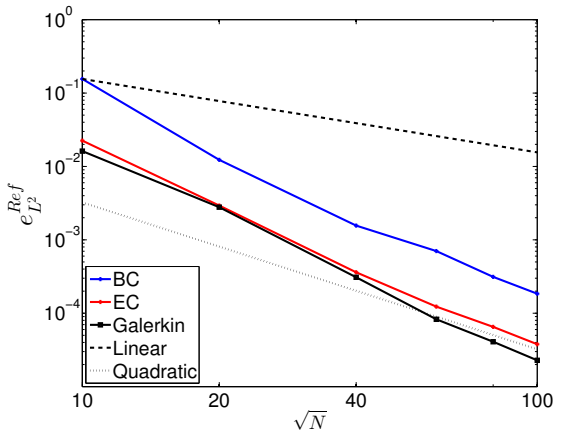

(j)

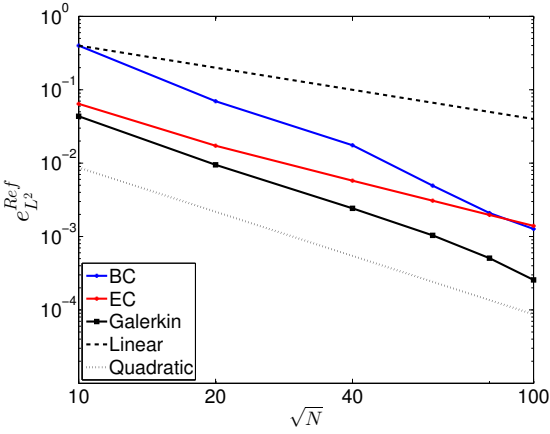

(b)

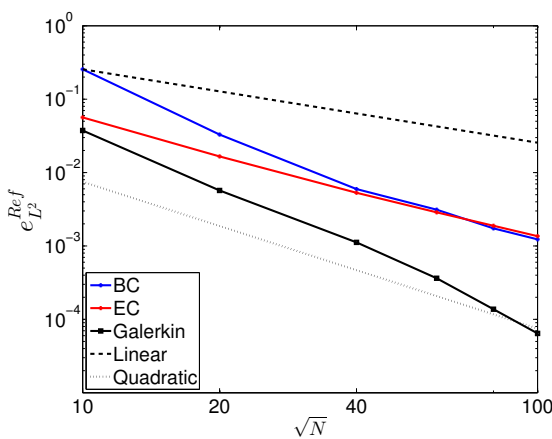

(e)

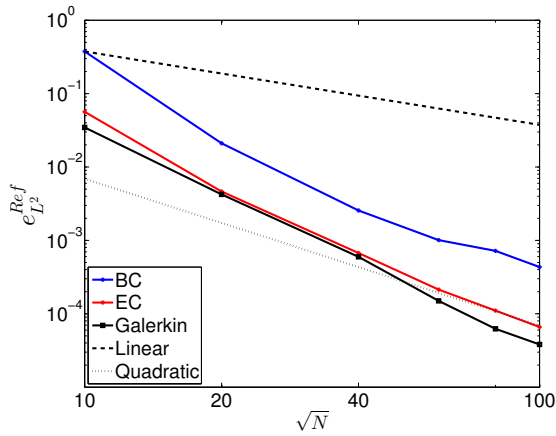

(h)

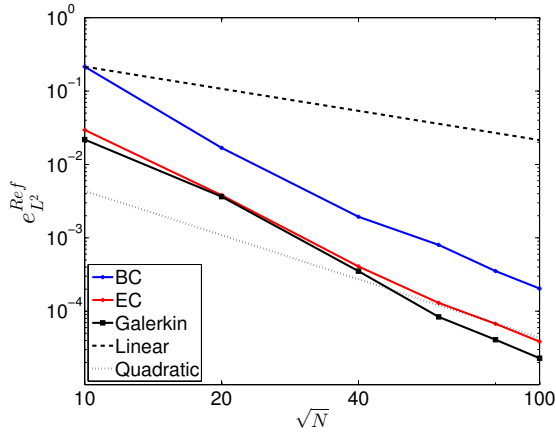

(k)

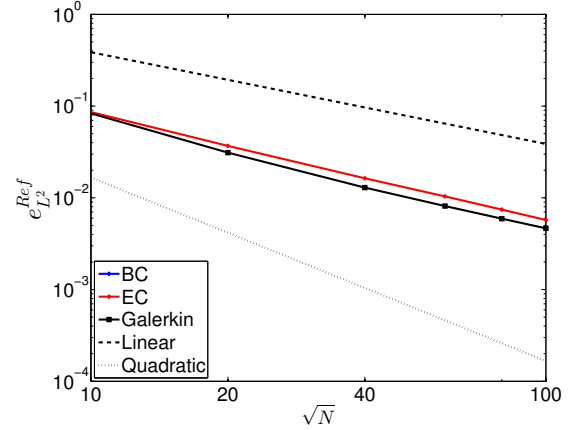

(c)

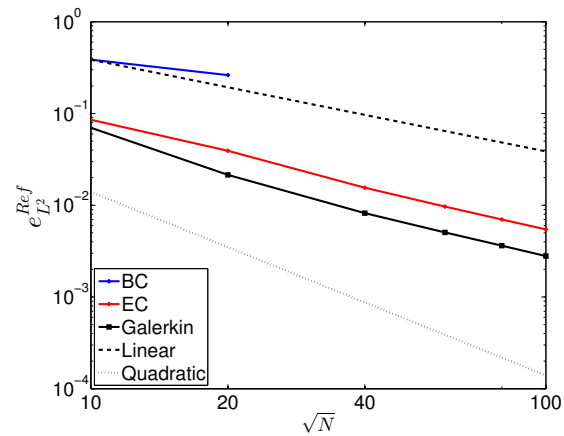

(f)

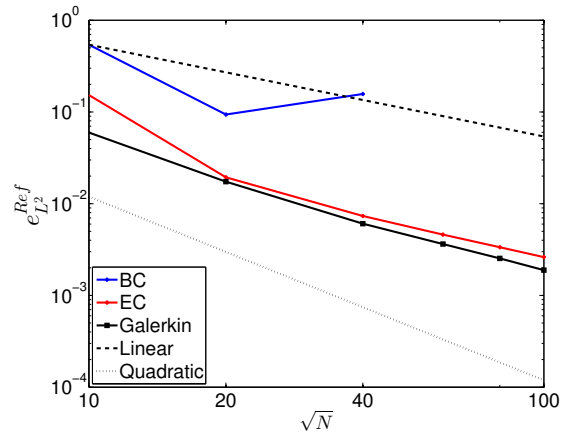

(i)

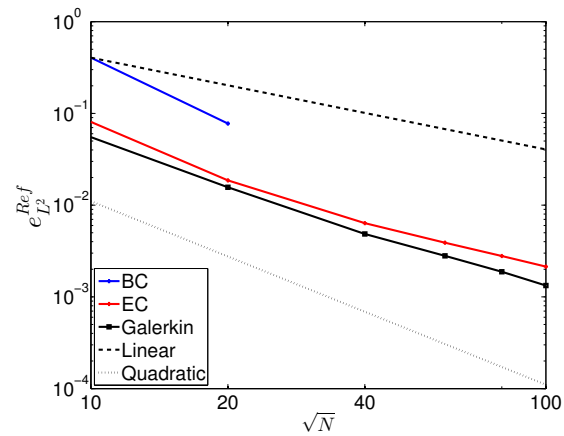

(l)

Figure 5: Example E2: Spatial convergence $\left(\mathrm{EC}\right.$ with $\left.C^{*}=4\right)$ for $(\mathrm{a}, \mathrm{d}, \mathrm{g}, \mathrm{j}) \hat{v}=0.25,(\mathrm{~b}, \mathrm{e}, \mathrm{h}, \mathrm{k}) \hat{v}=0.50$, and $(\mathrm{c}, \mathrm{f}, \mathrm{i}, \mathrm{l}) \hat{v}=-0.20$ with discretization order $2(\mathrm{a}, \mathrm{b}, \mathrm{c}), 3(\mathrm{~d}, \mathrm{e}, \mathrm{f}), 4(\mathrm{~g}, \mathrm{~h}, \mathrm{i}), 5(\mathrm{j}, \mathrm{k}, \mathrm{l})$. No-split Neo-Hookean model and non-uniform meshes (1:4 aspect ratio). 


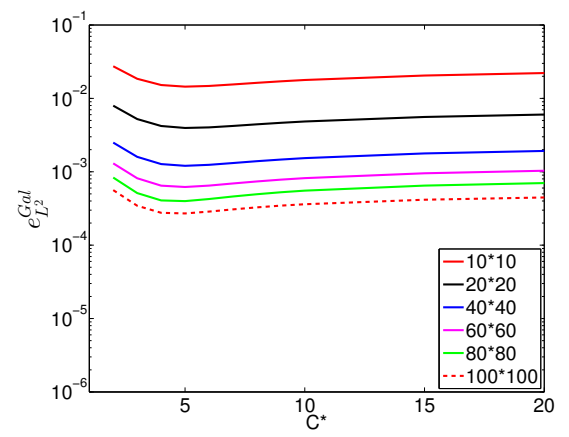

(a)

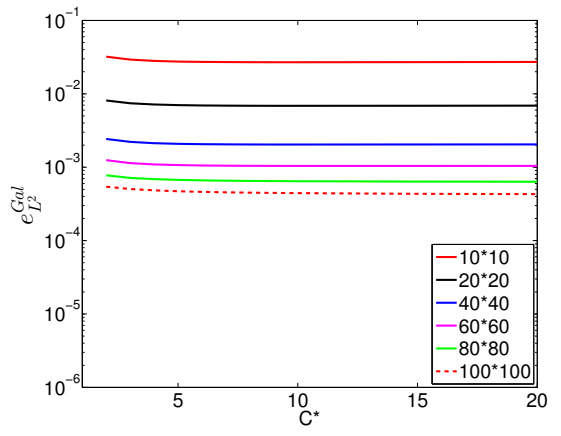

(d)

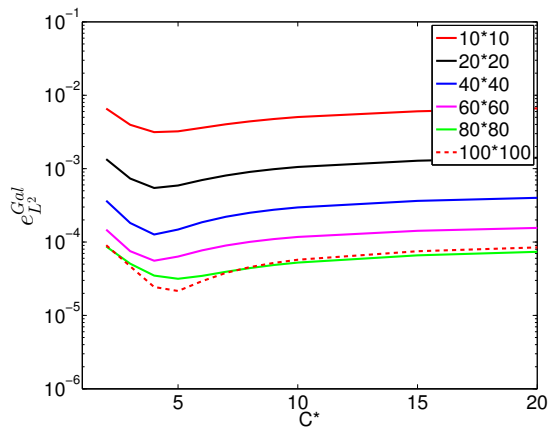

(g)

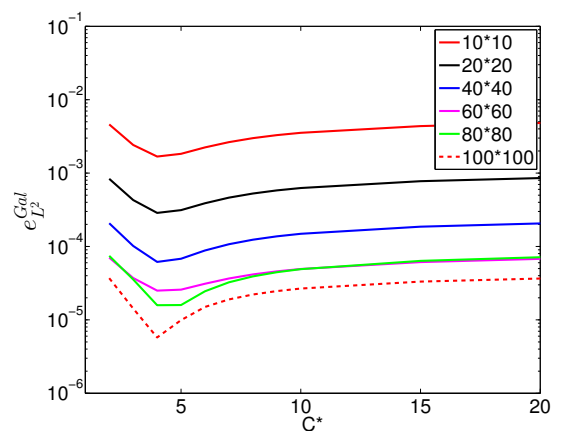

(j)

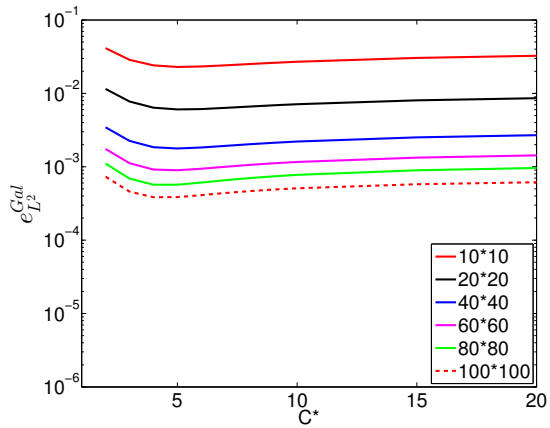

(b)

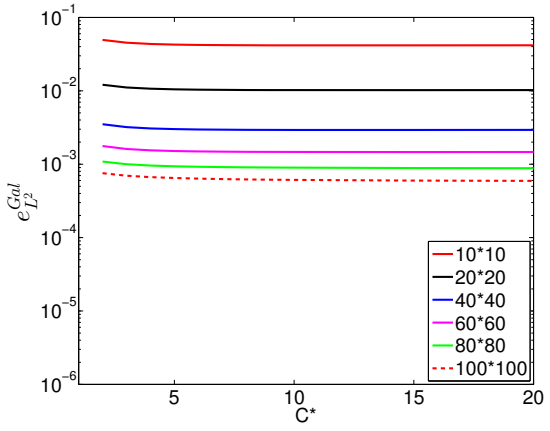

(e)

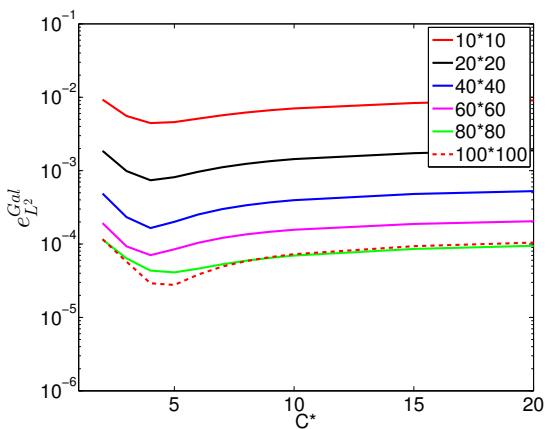

(h)

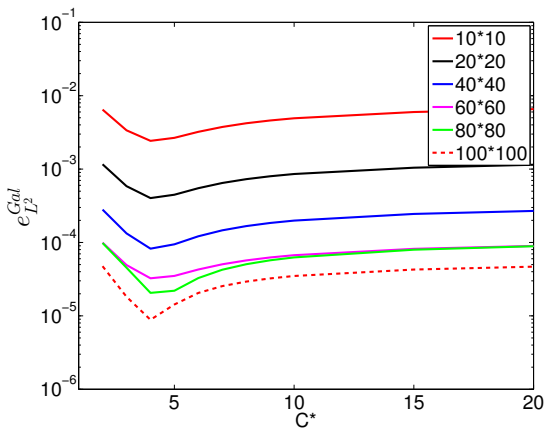

(k)

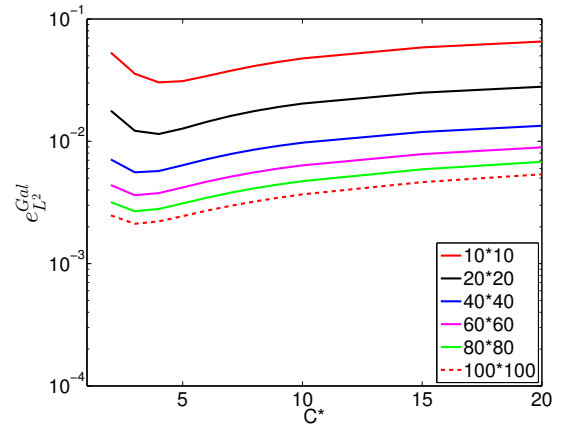

(c)

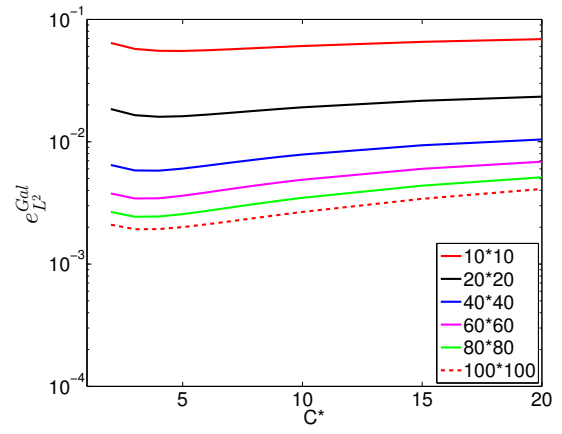

(f)

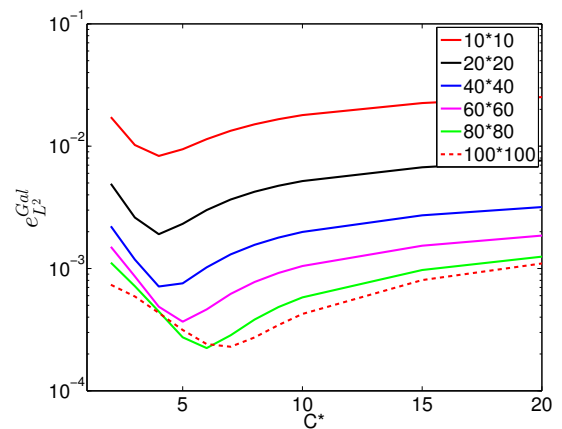

(i)

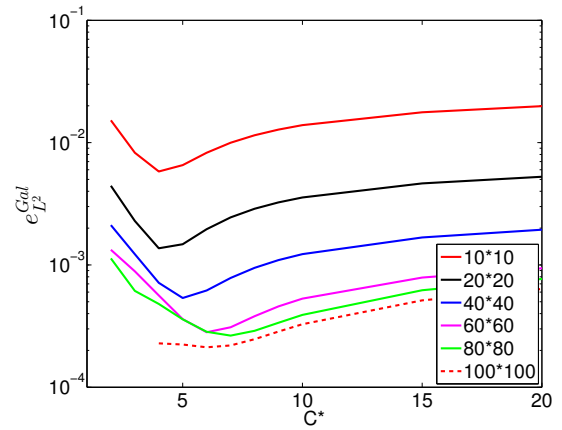

(1)

Figure 6: Example E2: Effect of $C^{*}$ for $(\mathrm{a}, \mathrm{d}, \mathrm{g}, \mathrm{j}) \hat{v}=0.25$, (b,e,h,k) $\hat{v}=0.50$, and $(\mathrm{c}, \mathrm{f}, \mathrm{i}, \mathrm{l}) \hat{v}=-0.20$ with discretization order 2 $(\mathrm{a}, \mathrm{b}, \mathrm{c}), 3(\mathrm{~d}, \mathrm{e}, \mathrm{f}), 4(\mathrm{~g}, \mathrm{~h}, \mathrm{i}), 5(\mathrm{j}, \mathrm{k}, \mathrm{l})$. No-split Neo-Hookean model and uniform meshes. 


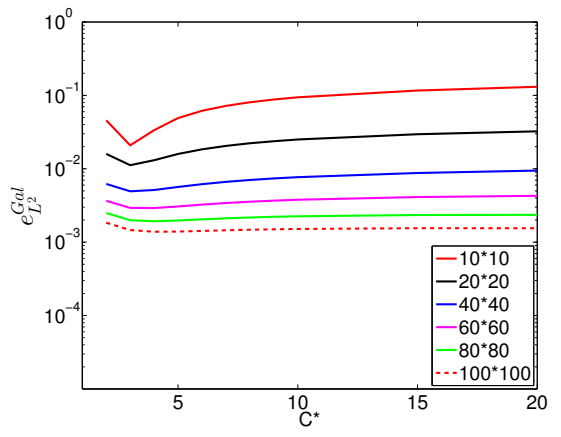

(a)

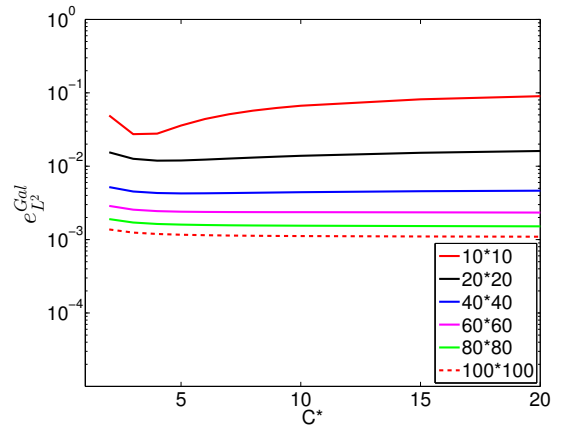

(d)

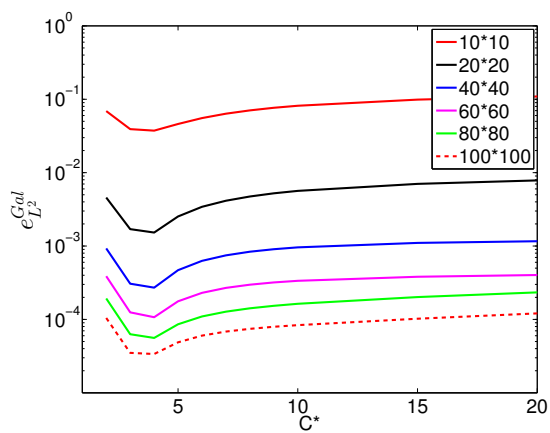

(g)

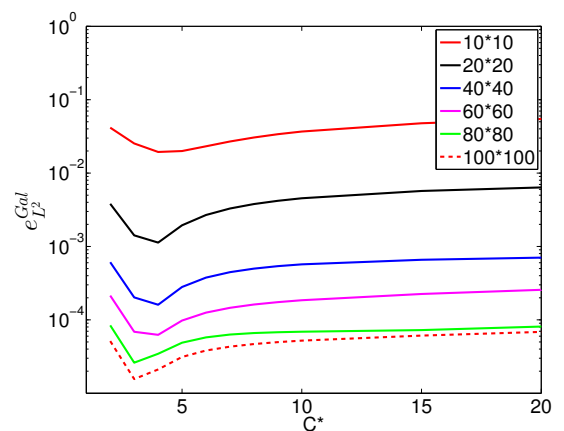

(j)

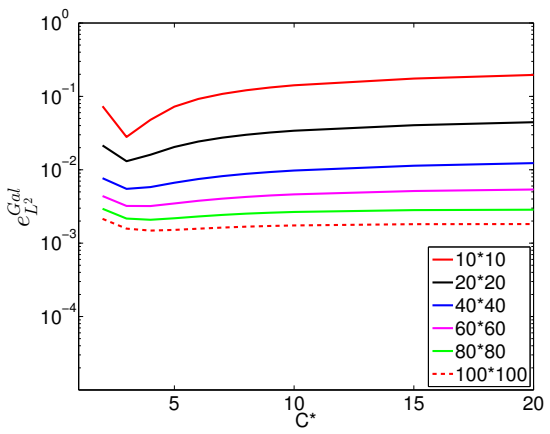

(b)

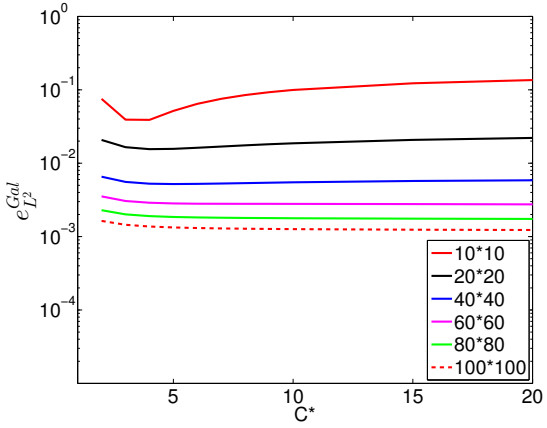

(e)

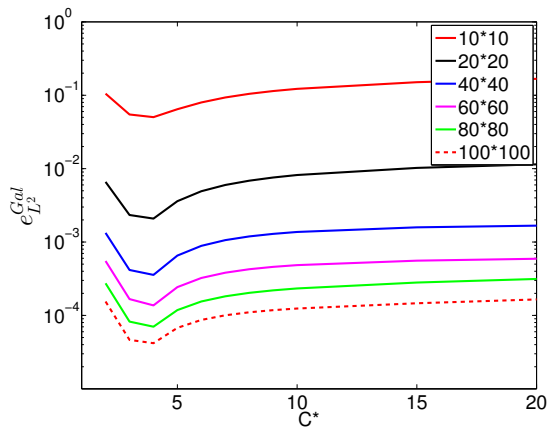

(h)

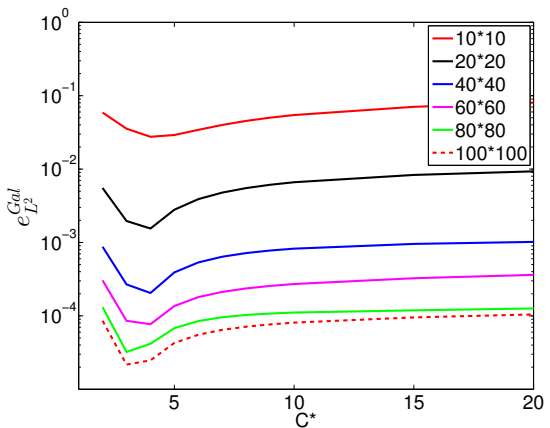

(k)

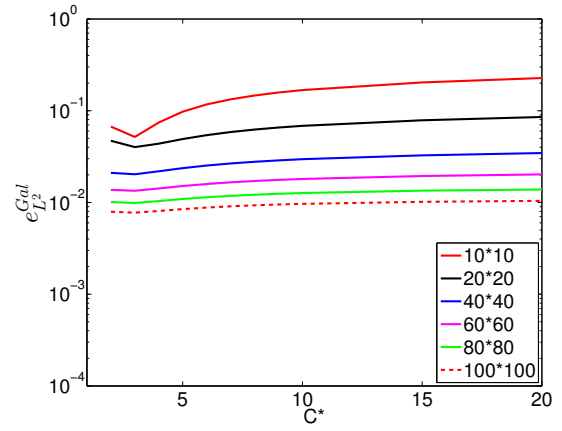

(c)

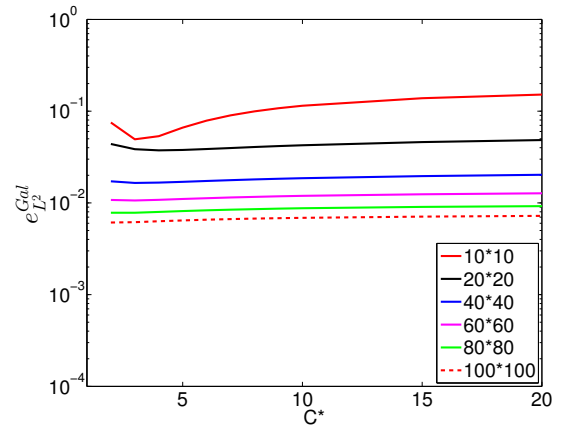

(f)

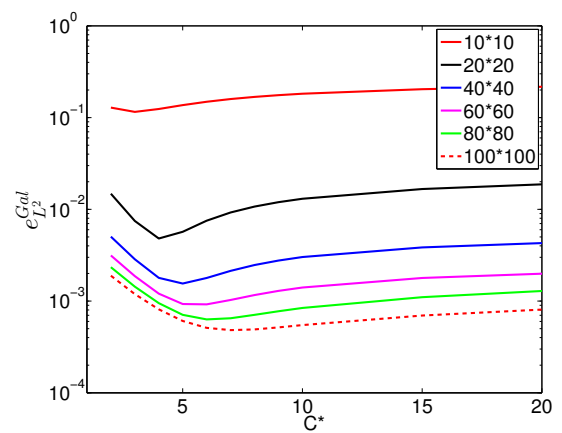

(i)

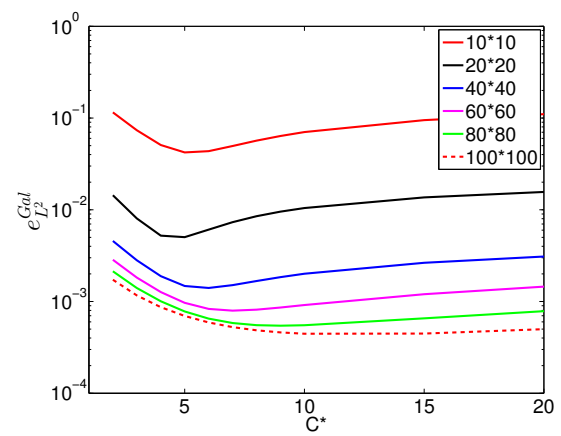

(1)

Figure 7: Example E2: Effect of $C^{*}$ for $(\mathrm{a}, \mathrm{d}, \mathrm{g}, \mathrm{j}) \hat{v}=0.25$, (b,e,h,k) $\hat{v}=0.50$, and $(\mathrm{c}, \mathrm{f}, \mathrm{i}, \mathrm{l}) \hat{v}=-0.20$ with discretization order 2 $(\mathrm{a}, \mathrm{b}, \mathrm{c}), 3$ (d,e,f), 4 (g,h,i), 5 (j,k,l). No-split Neo-Hookean model and non-uniform meshes (1:4 aspect ratio). 


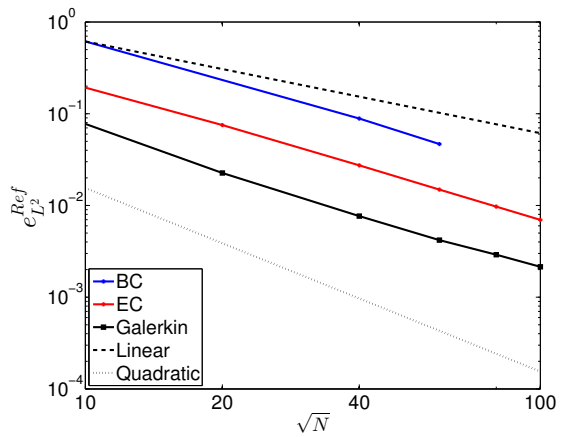

(a)

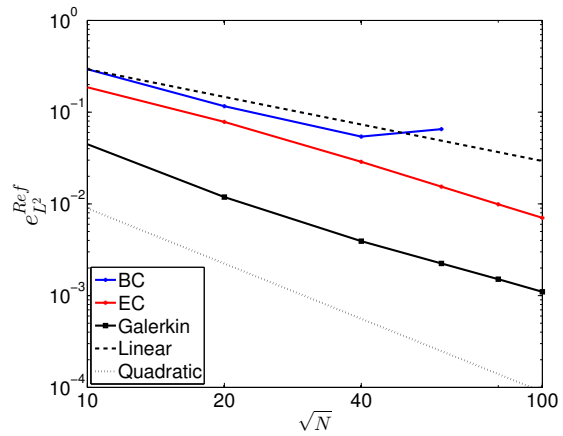

(c)

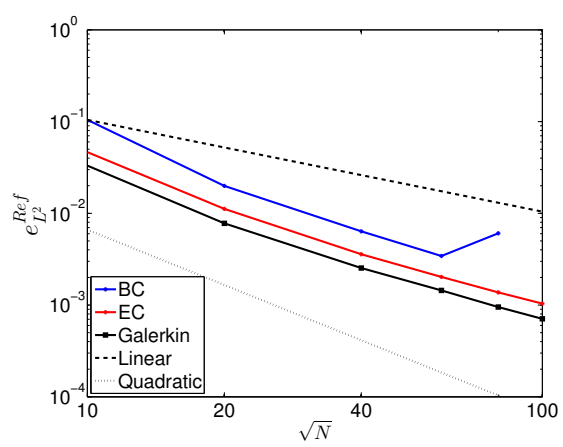

(e)

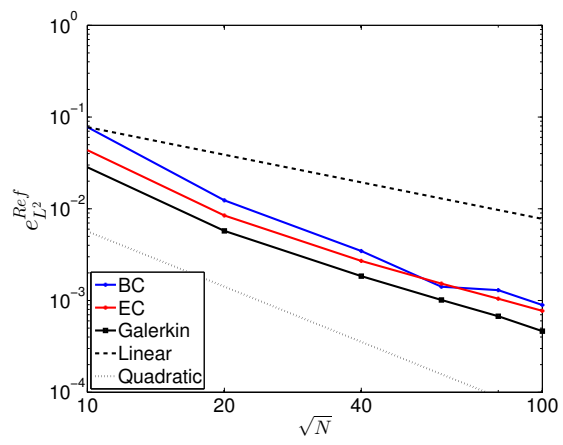

(g)

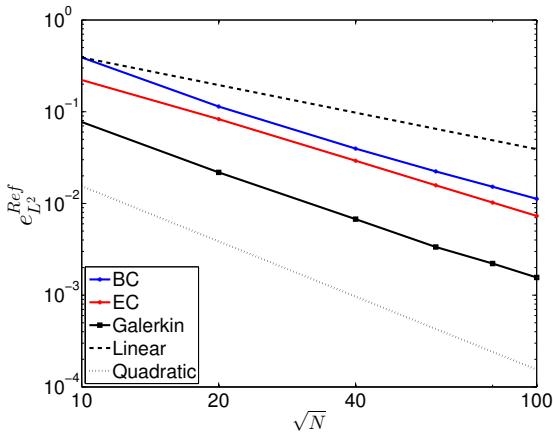

(b)

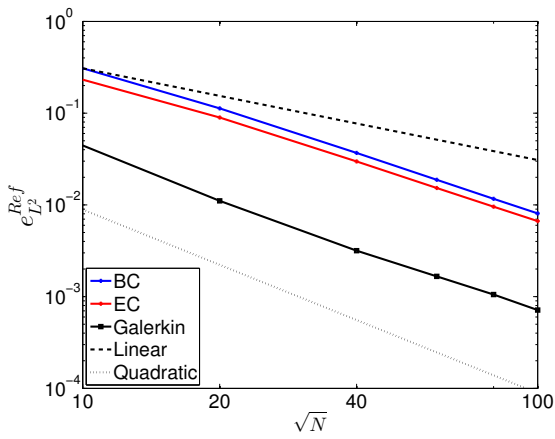

(d)

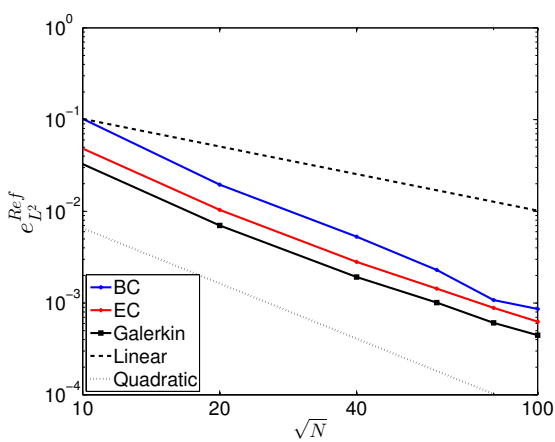

(f)

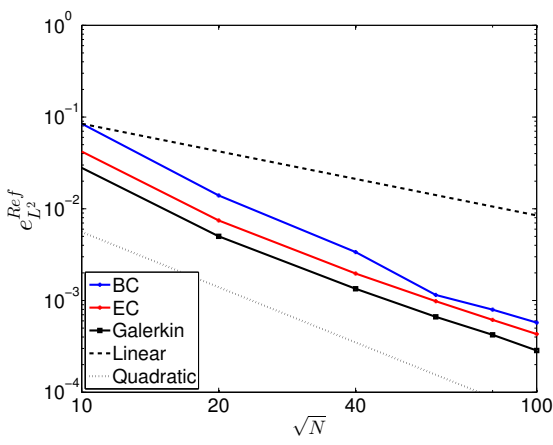

(h)

Figure 8: Example E3: Spatial convergence (EC with $\left.C^{*}=4\right)$. (a,c,e,g) No-split Neo-Hookean model, (b,d,f,h) split NeoHookean model with discretization order $2(\mathrm{a}, \mathrm{b}), 3(\mathrm{c}, \mathrm{d}), 4(\mathrm{e}, \mathrm{f}), 5(\mathrm{~g}, \mathrm{~h})$. Uniform meshes. 


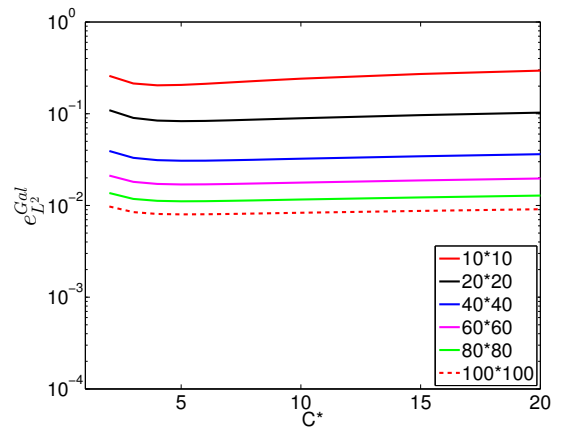

(a)

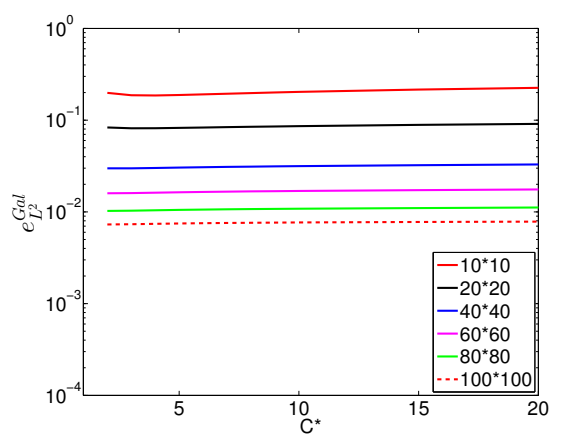

(c)

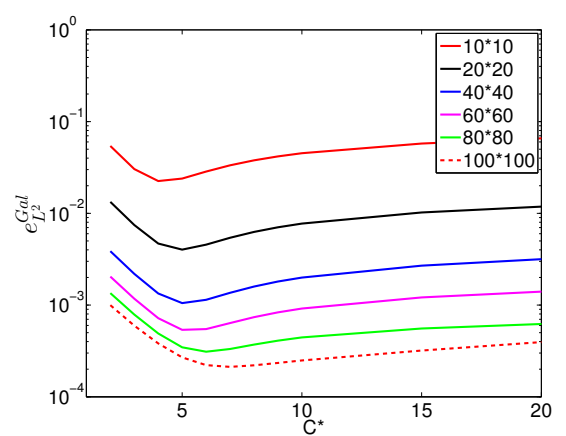

(e)

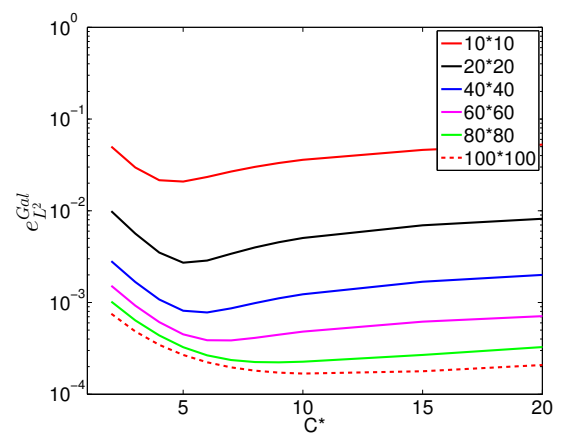

(g)

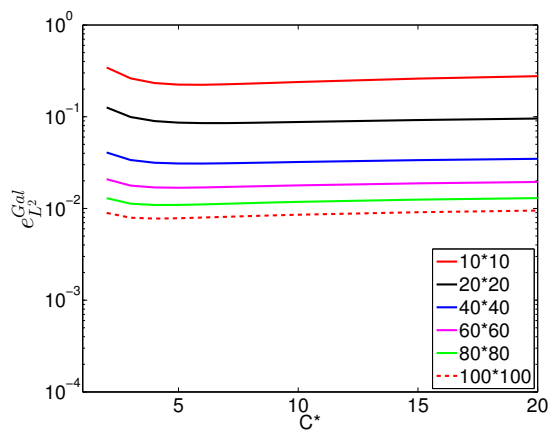

(b)

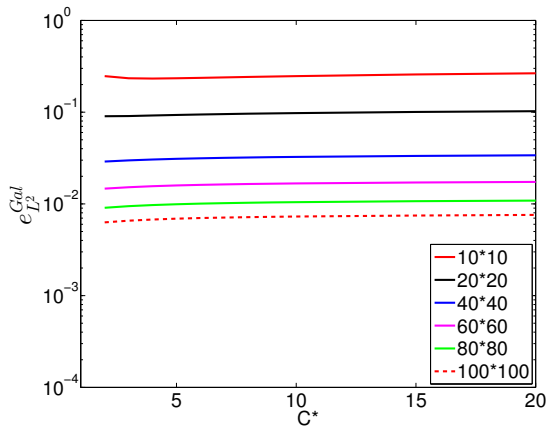

(d)

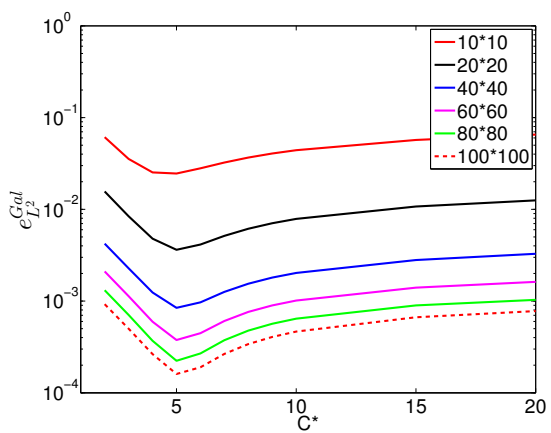

(f)

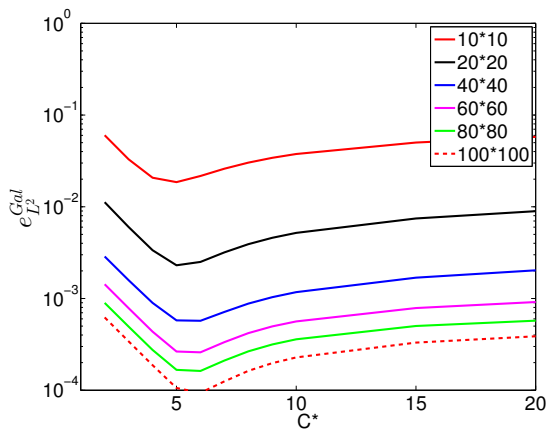

(h)

Figure 9: Example E3: Choice of $C^{*}$. (a,c,e,g) No-split Neo-Hookean model, (b,d,f,h) split Neo-Hookean model with discretization order $2(\mathrm{a}, \mathrm{b}), 3(\mathrm{c}, \mathrm{d}), 4(\mathrm{e}, \mathrm{f}), 5(\mathrm{~g}, \mathrm{~h})$. Uniform meshes. 


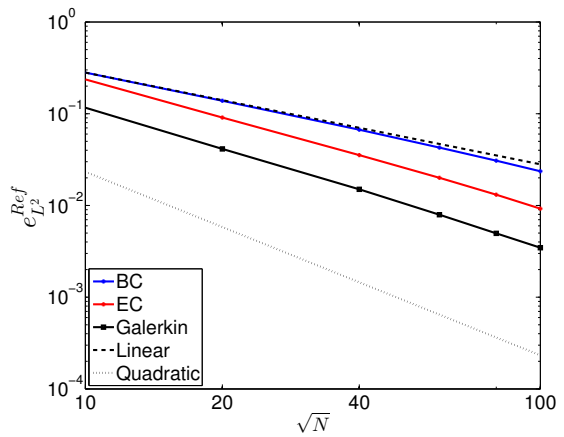

(a)

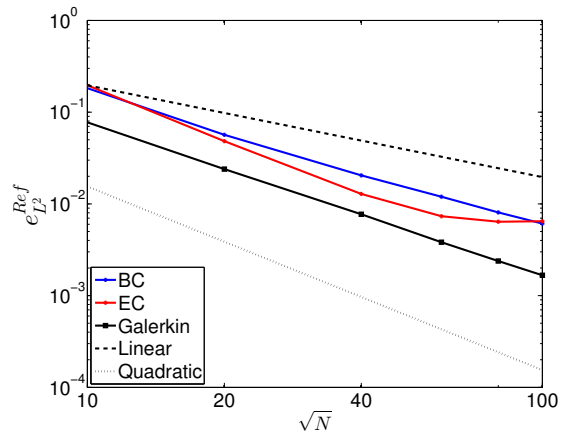

(c)

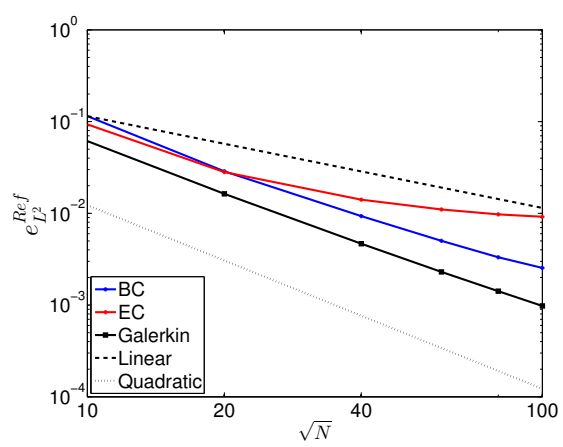

(e)

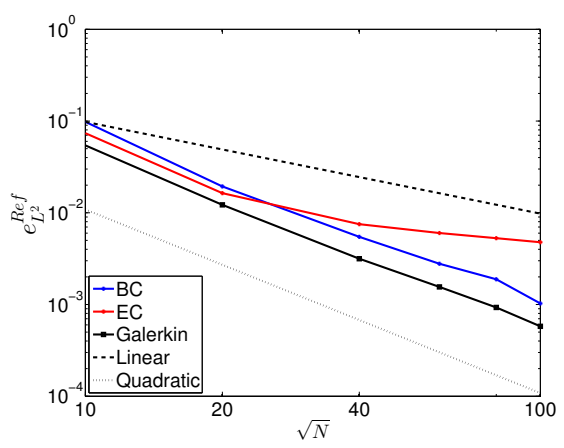

(g)

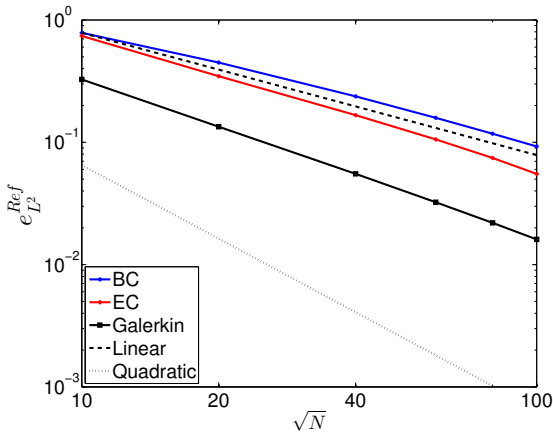

(b)

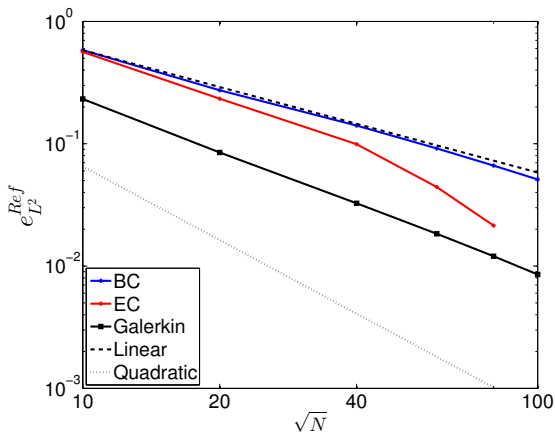

(d)

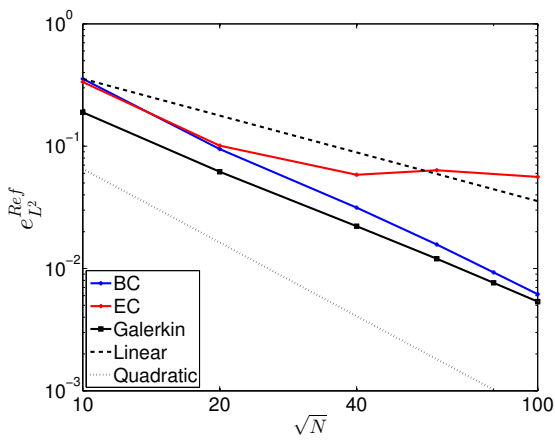

(f)

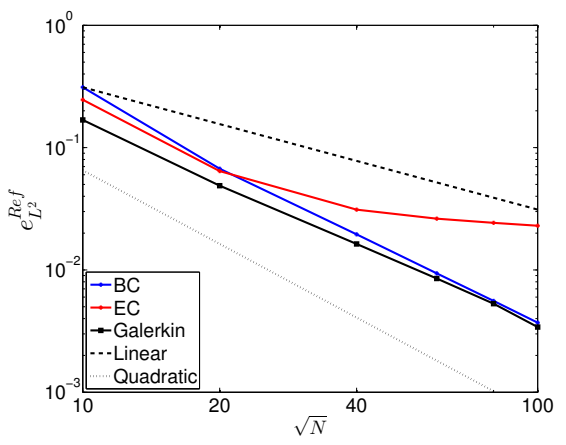

(h)

Figure 10: Example E4: Spatial convergence (EC with $\left.C^{*}=4\right)$. (a,c,e,g) Barrel-shape $1\left(136^{\circ}\right),(\mathrm{b}, \mathrm{d}, \mathrm{f}, \mathrm{h})$ barrel-shape $2\left(154^{\circ}\right)$ with discretization order $2(\mathrm{a}, \mathrm{b}), 3(\mathrm{c}, \mathrm{d}), 4(\mathrm{e}, \mathrm{f}), 5(\mathrm{~g}, \mathrm{~h})$. No-split Neo-Hookean model and uniform meshes. 


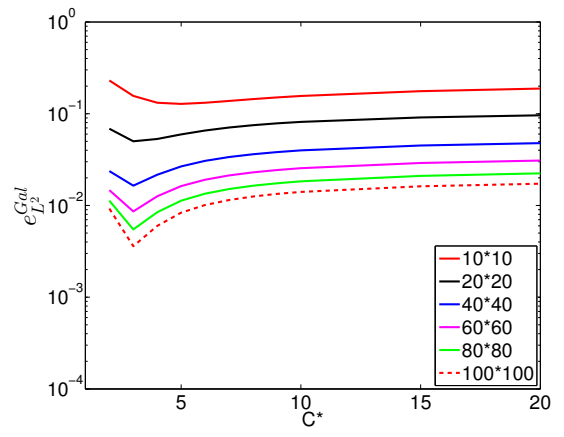

(a)

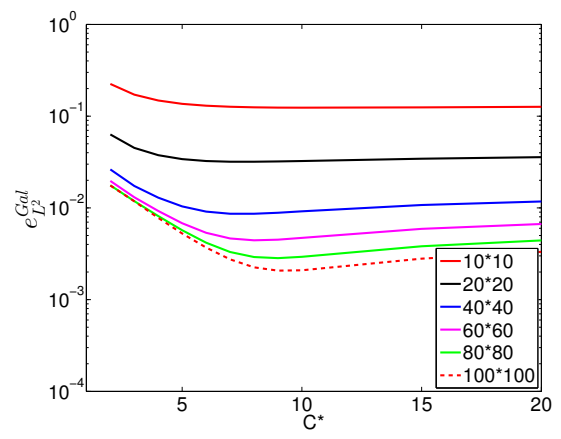

(c)

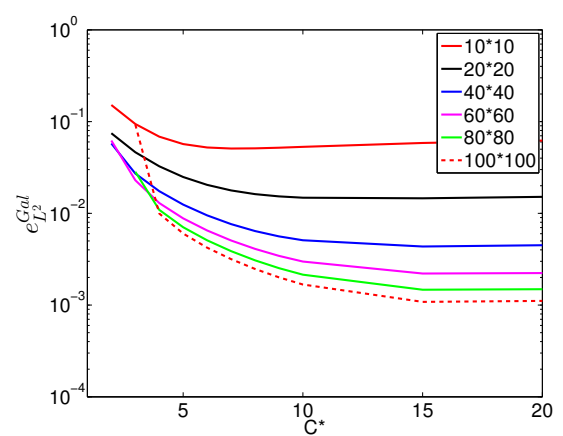

(e)

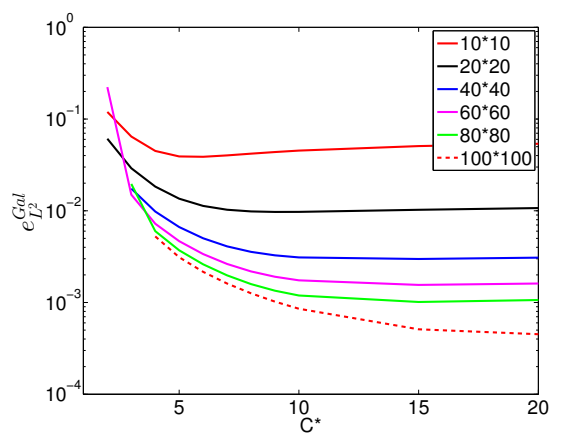

(g)

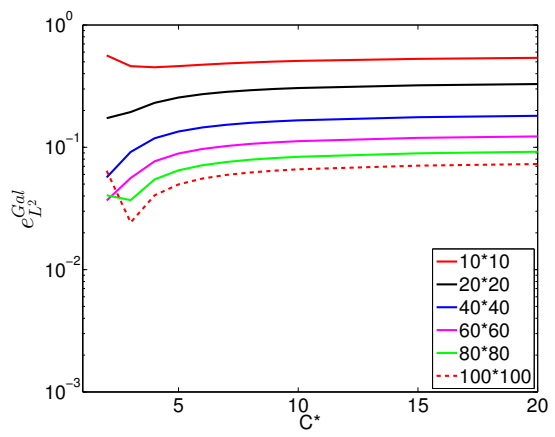

(b)

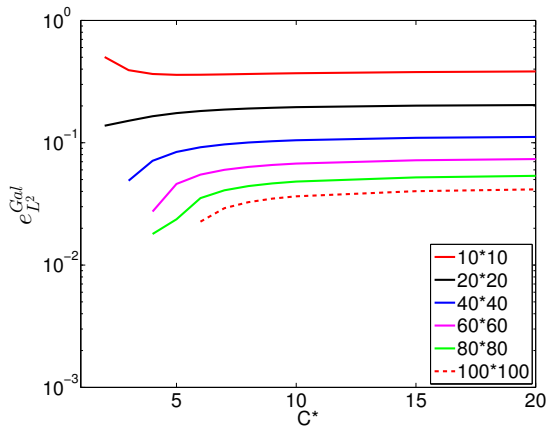

(d)

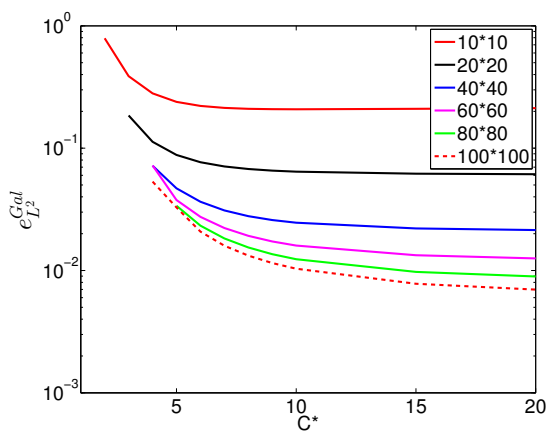

(f)

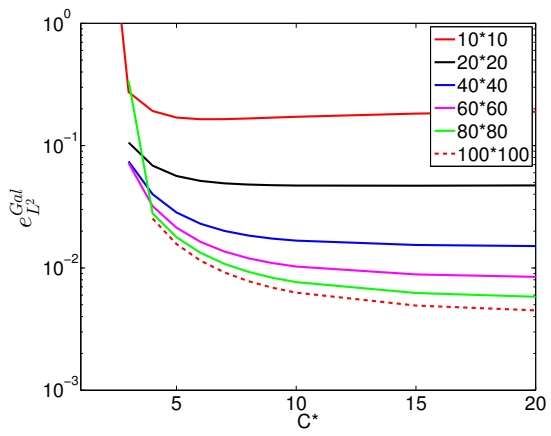

(h)

Figure 11: Example E4: Choice of $C^{*}$. (a,c,e,g) Barrel-shape $1\left(136^{\circ}\right),(\mathrm{b}, \mathrm{d}, \mathrm{f}, \mathrm{h})$ barrel-shape $2\left(154^{\circ}\right)$ with discretization order $2(\mathrm{a}, \mathrm{b}), 3(\mathrm{c}, \mathrm{d}), 4(\mathrm{e}, \mathrm{f}), 5$ (g,h). No-split Neo-Hookean model and uniform meshes. 


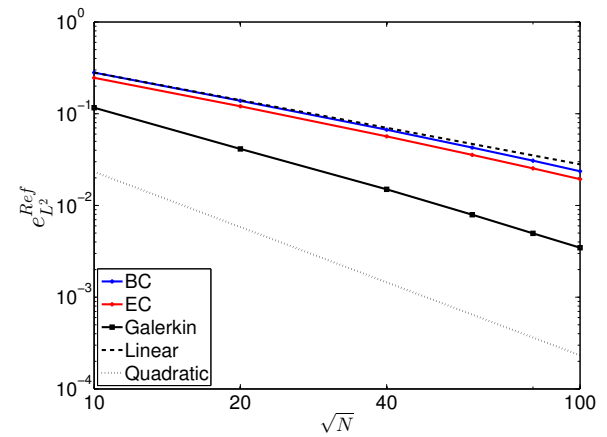

(a)

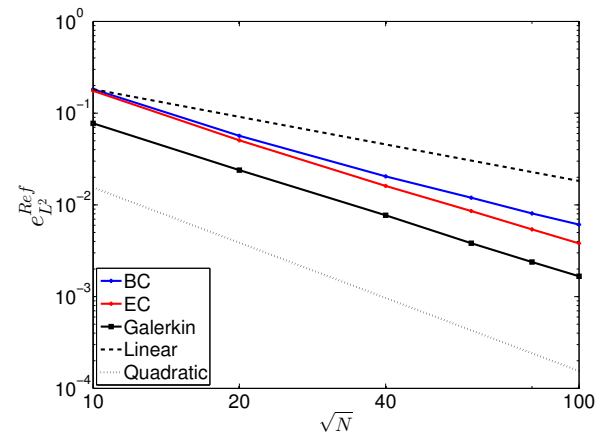

(c)

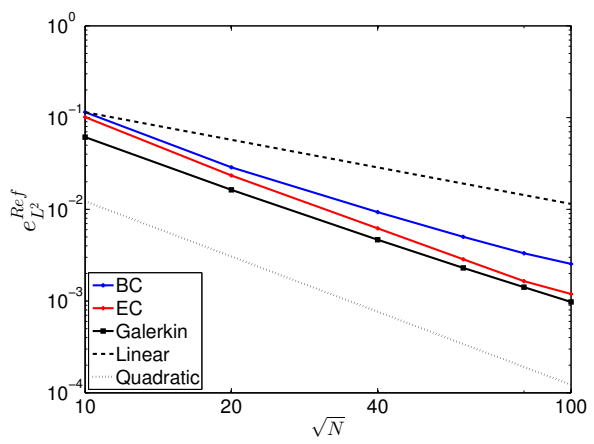

(e)

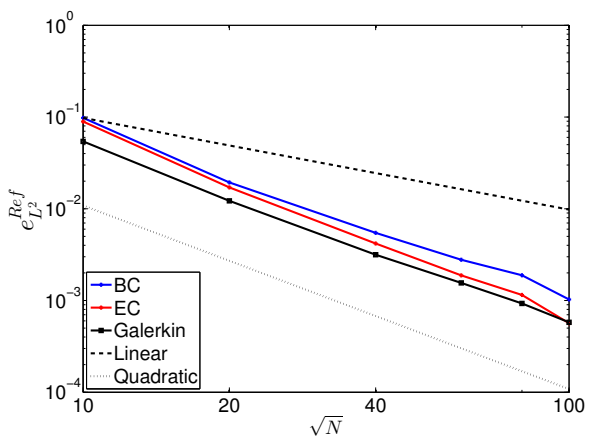

(g)

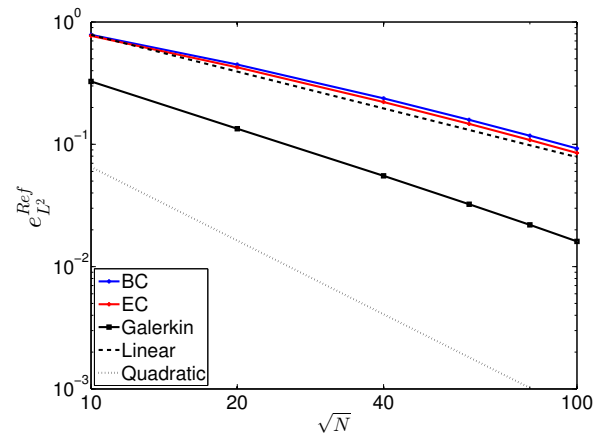

(b)

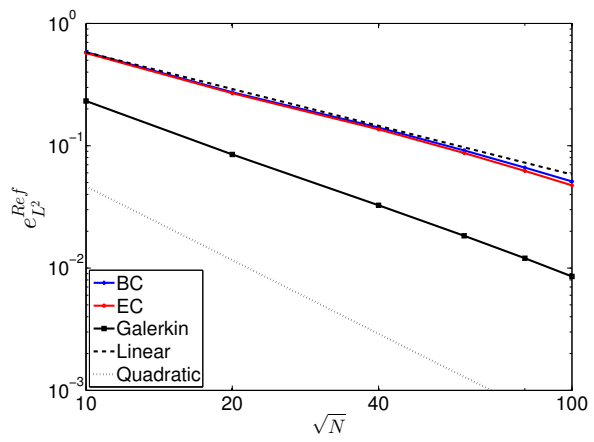

(d)

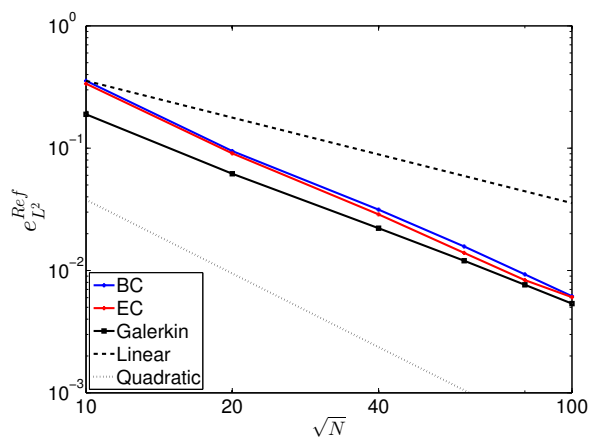

(f)

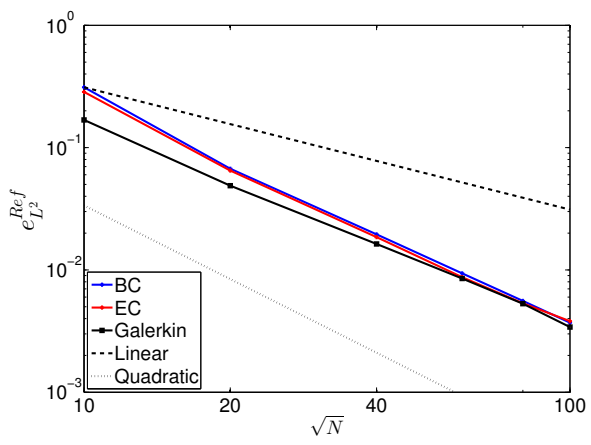

(h)

Figure 12: Example E4: Spatial convergence (EC with $\left.C^{*}=15\right)$. (a,c,e,g) Barrel-shape $1\left(136^{\circ}\right),(\mathrm{b}, \mathrm{d}, \mathrm{f}, \mathrm{h})$ barrel-shape $2\left(154^{\circ}\right)$ with discretization order $2(\mathrm{a}, \mathrm{b}), 3(\mathrm{c}, \mathrm{d}), 4(\mathrm{e}, \mathrm{f}), 5(\mathrm{~g}, \mathrm{~h})$. No-split Neo-Hookean model and uniform meshes. 


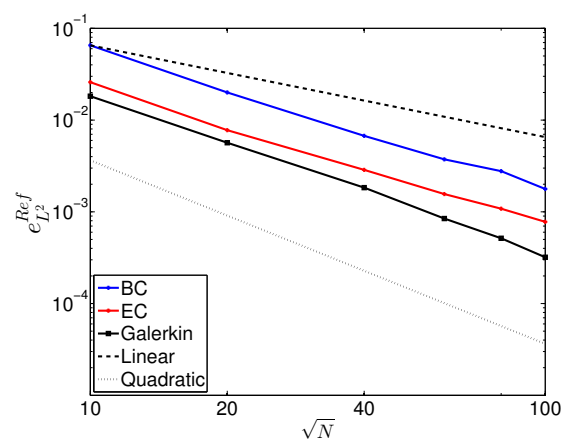

(a)

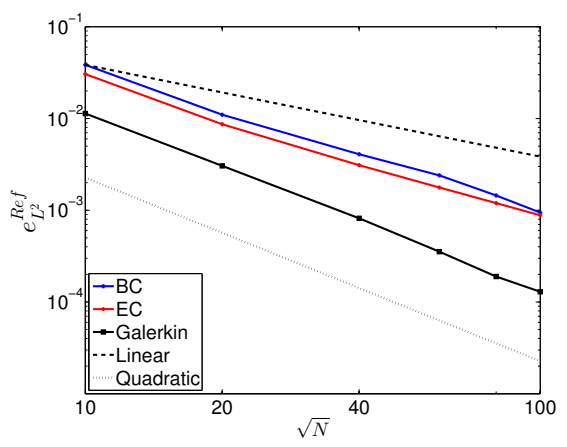

(d)

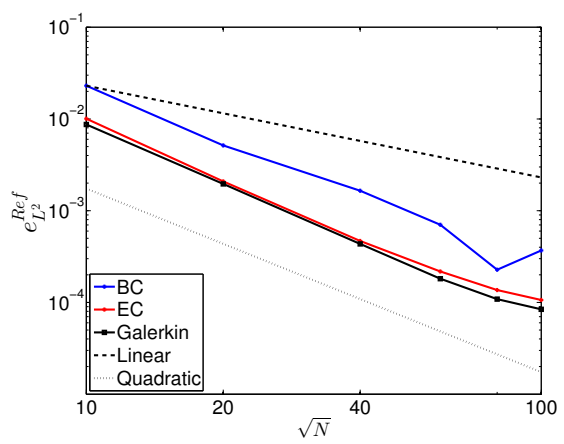

(g)

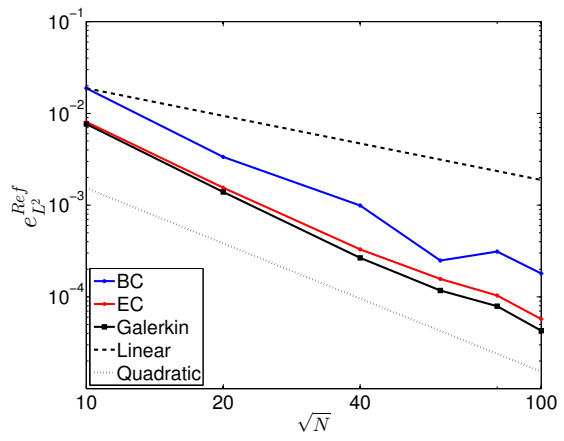

(j)

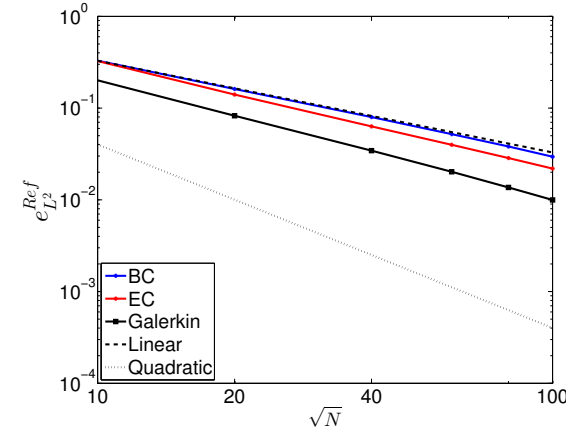

(b)

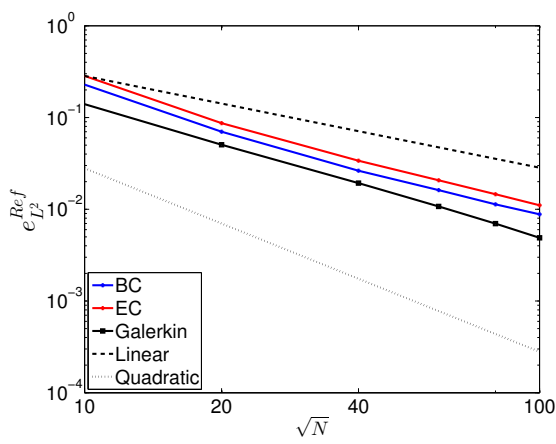

(e)

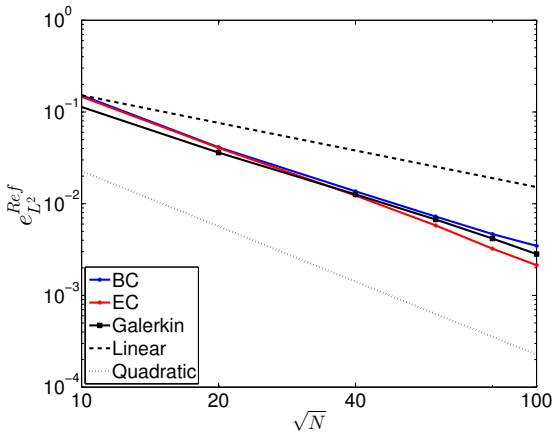

(h)

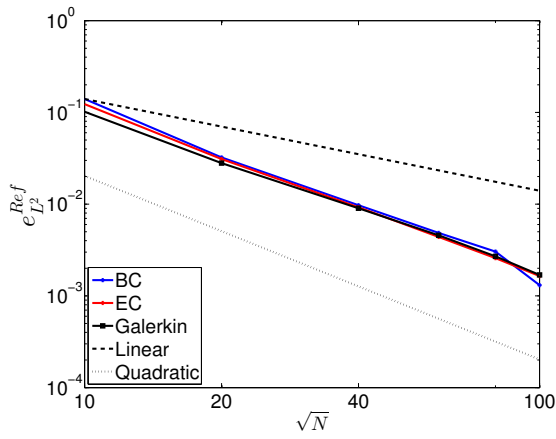

(k)

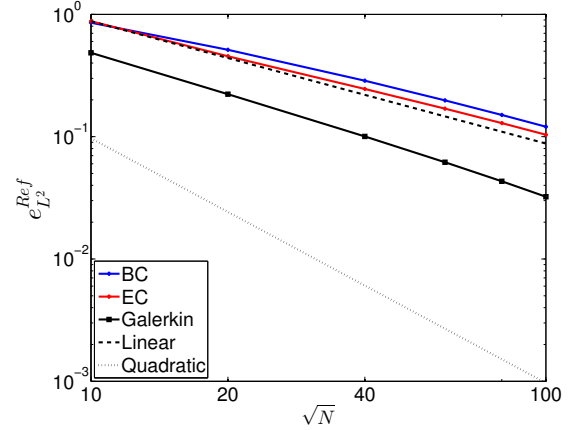

(c)

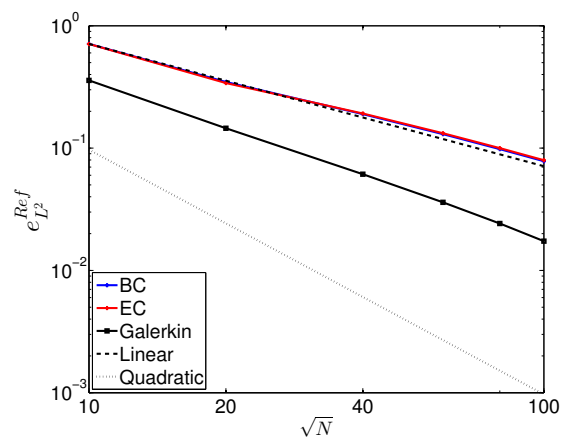

(f)

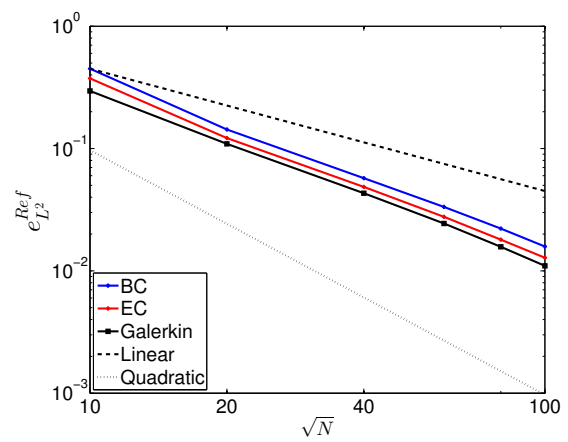

(i)

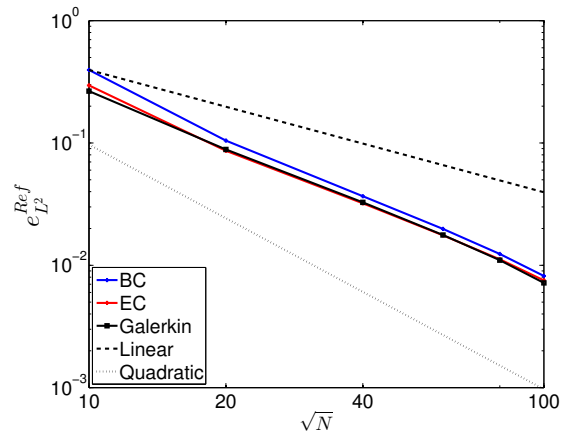

(l)

Figure 13: Example E4: Spatial convergence (EC with $\left.C^{*}=4\right)$. (a,d,g,j) Square, (b,e,h,k) barrel-shape $1\left(136^{\circ}\right),(\mathrm{c}, \mathrm{f}, \mathrm{i}, \mathrm{l})$ barrel-shape $2\left(154^{\circ}\right)$ with discretization order $2(\mathrm{a}, \mathrm{b}, \mathrm{c}), 3(\mathrm{~d}, \mathrm{e}, \mathrm{f}), 4(\mathrm{~g}, \mathrm{~h}, \mathrm{i}), 5(\mathrm{j}, \mathrm{k}, \mathrm{l})$. Small deformation elasticity and uniform meshes. 


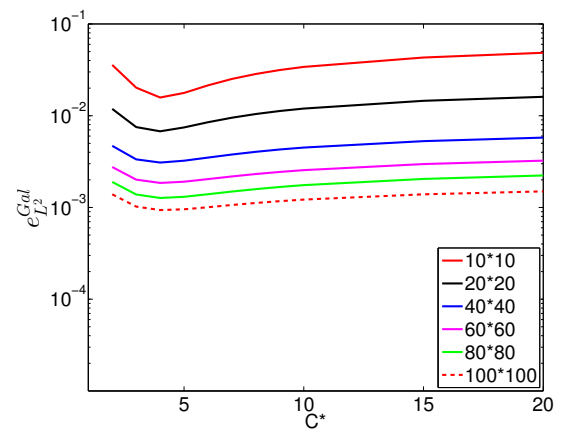

(a)

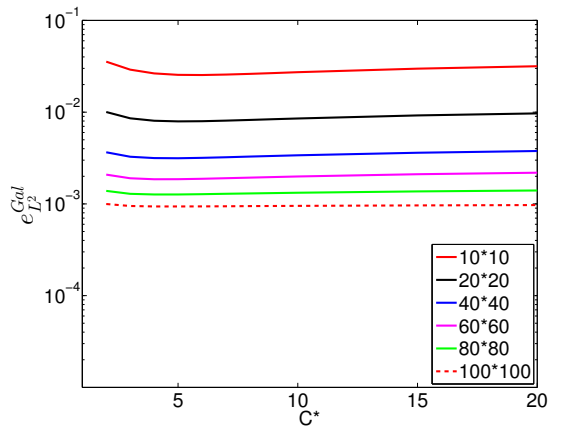

(d)

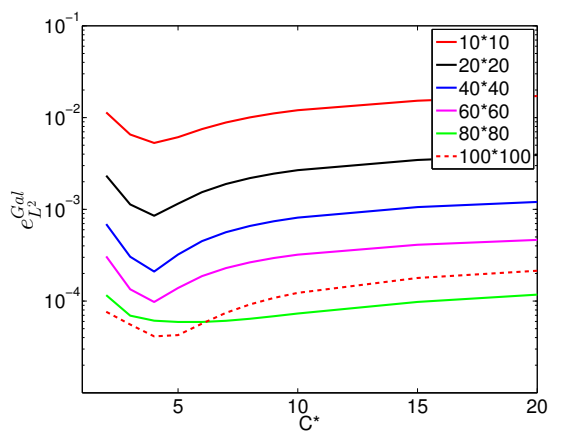

(g)

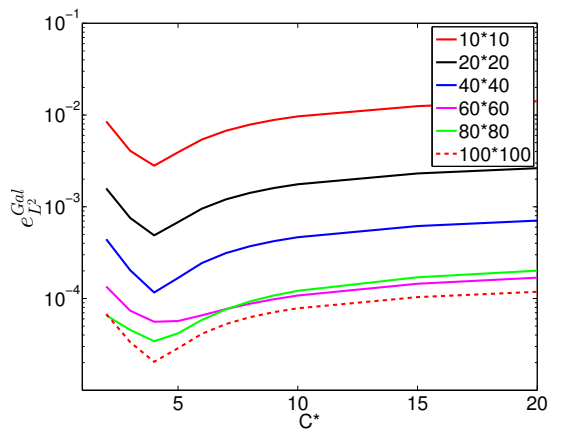

(j)

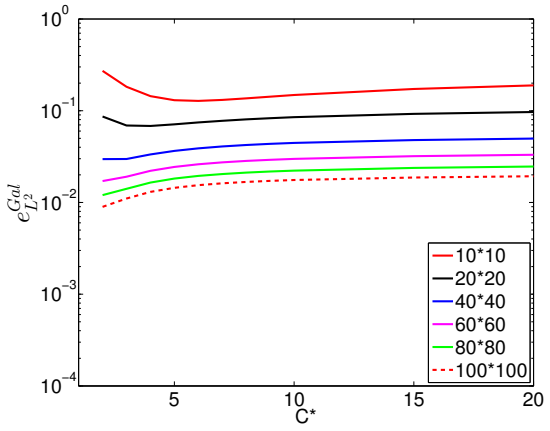

(b)

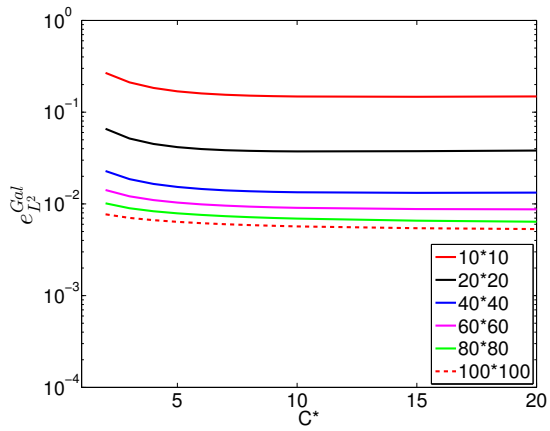

(e)

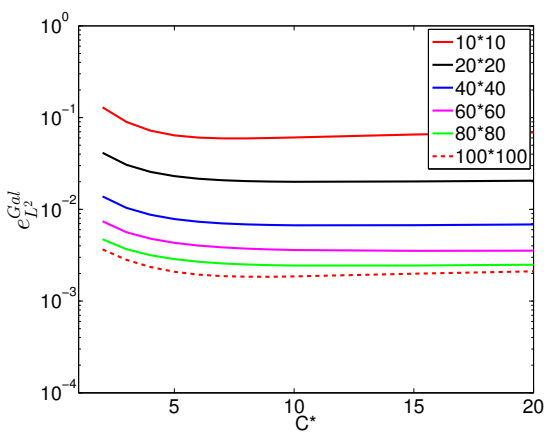

(h)

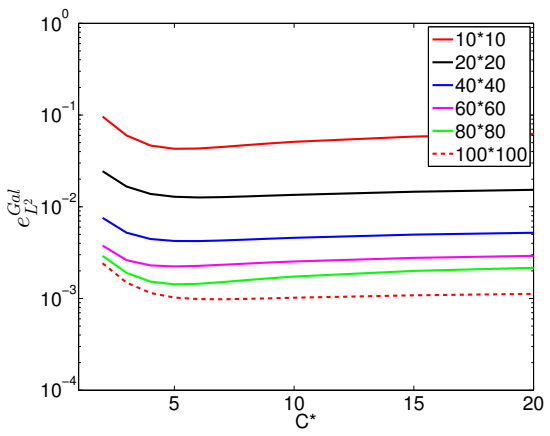

(k)

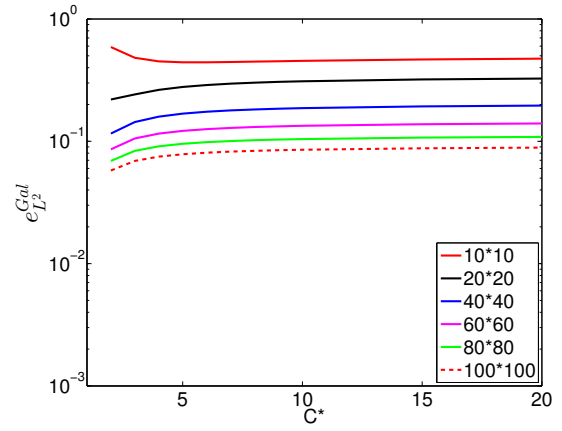

(c)

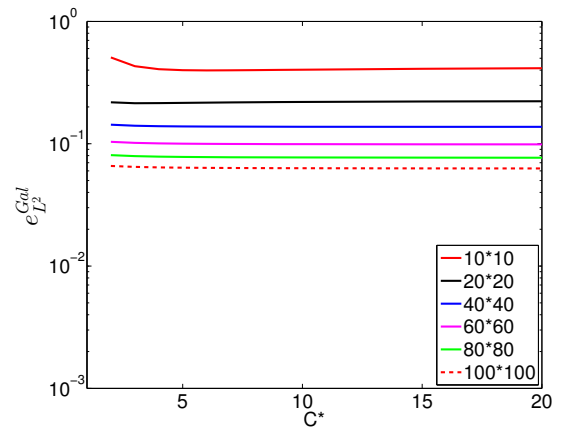

(f)

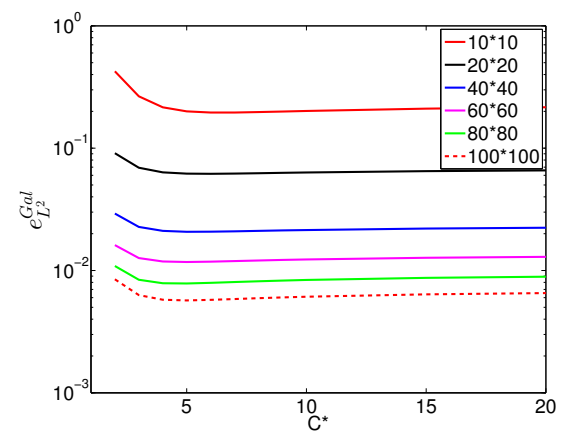

(i)

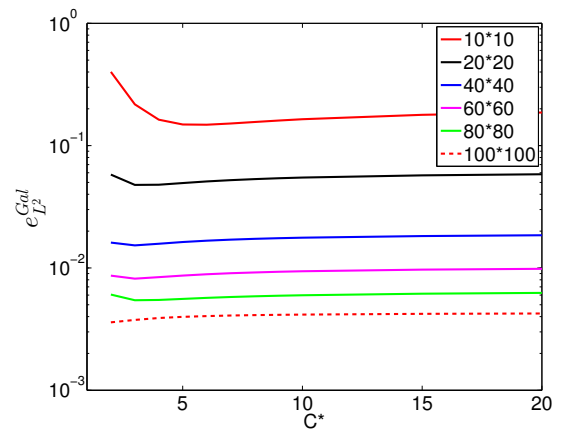

(l)

Figure 14: Example E4: Choice of $C^{*}$. (a,d,g,j) Square, (b,e,h,k) barrel-shape $1\left(136^{\circ}\right),(\mathrm{c}, \mathrm{f}, \mathrm{i}, \mathrm{l})$ barrel-shape $2\left(154^{\circ}\right)$ with discretization order $2(\mathrm{a}, \mathrm{b}, \mathrm{c}), 3(\mathrm{~d}, \mathrm{e}, \mathrm{f}), 4(\mathrm{~g}, \mathrm{~h}, \mathrm{i}), 5(\mathrm{j}, \mathrm{k}, \mathrm{l})$. Uniform meshes. Small deformation elasticity and uniform meshes. 


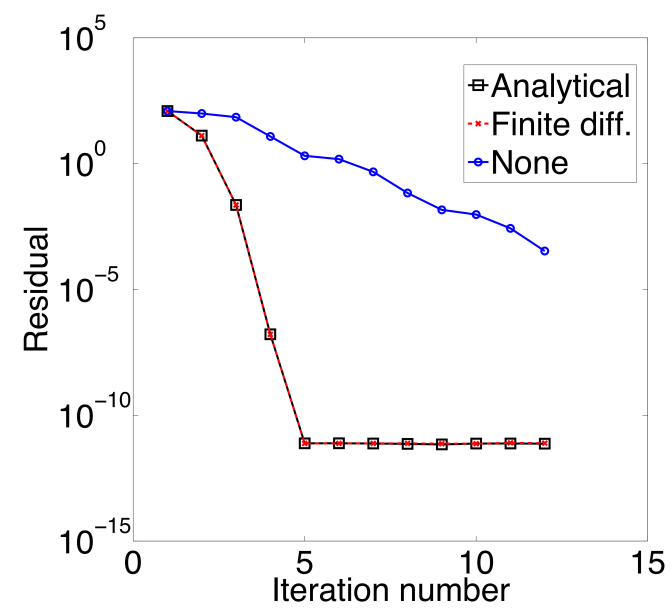

Figure 15: Example E2: Effect of the computation of $\mathbb{D}$ on the iterative convergence history. $p=2$, $40 \mathrm{x} 40$ mesh, EC with $C^{*}=4$. "None" indicates the $\mathbb{D}$ contribution to the tangent stiffness is omitted.

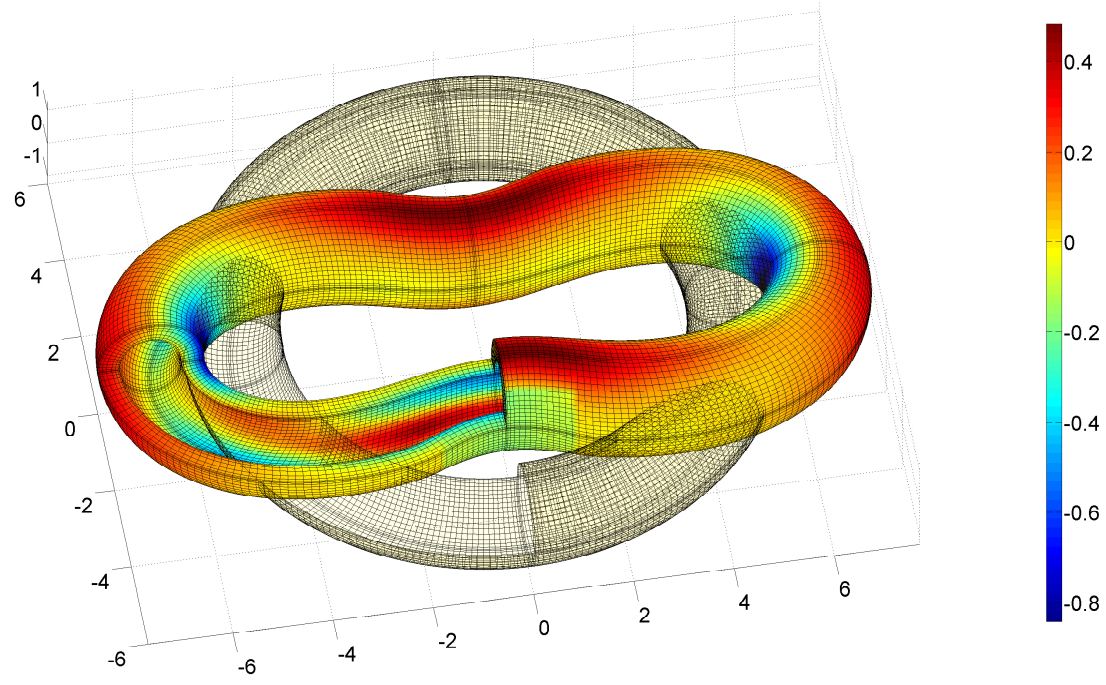

Figure 16: 3D example. A hollow torus of neo-Hookean material is subjected to internal pressure and pinched by applying vertical tractions to the central part of its outer surface. The contour illustrates the $1^{\text {st }}$ Piola-Kirchhoff stress in vertical (pinched) direction. The grid marks the position of the collocation points. Part of the surface is removed to show the stress contour in the interior. 


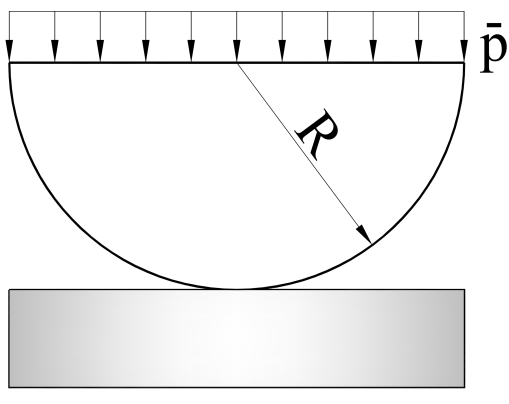

(a)

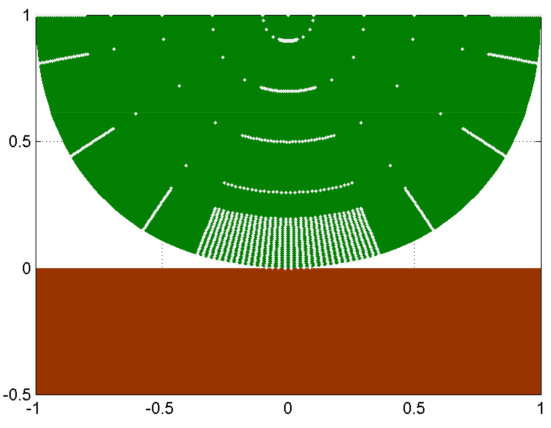

(b)

Figure 17: Example C1: (a) Setup. (b) Collocation points for the coarsest mesh.

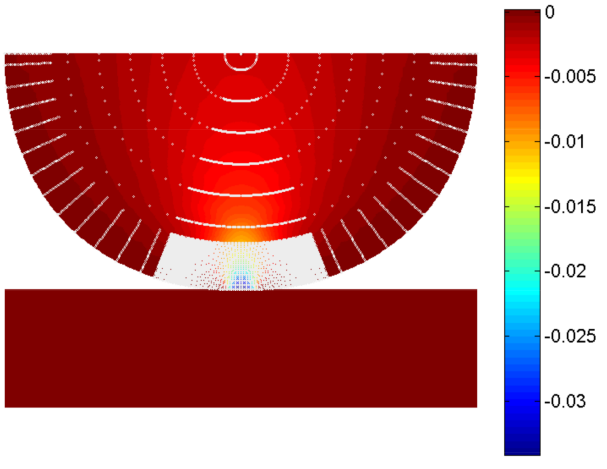

(a)

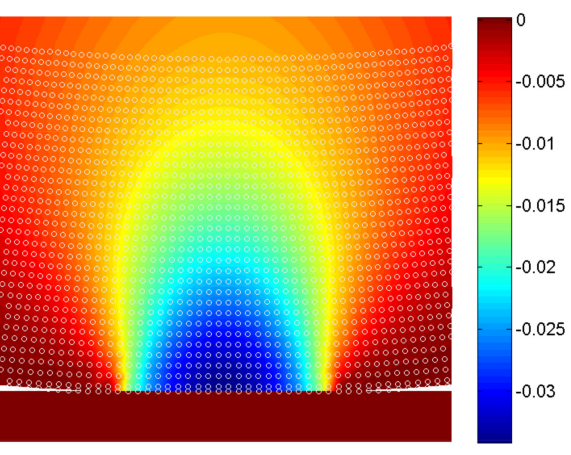

(b)

Figure 18: Example C1: (a) Contour plot of $\sigma_{y y}$. (b) Close-up of the contact region. Results obtained with BC. 


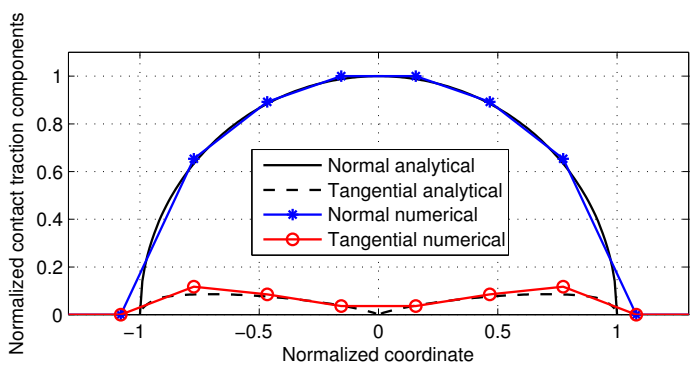

(a)

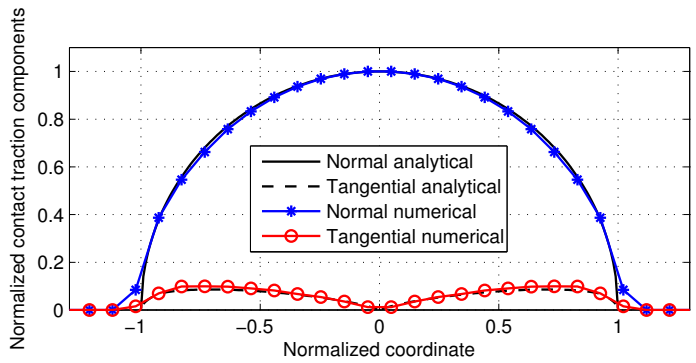

(c)

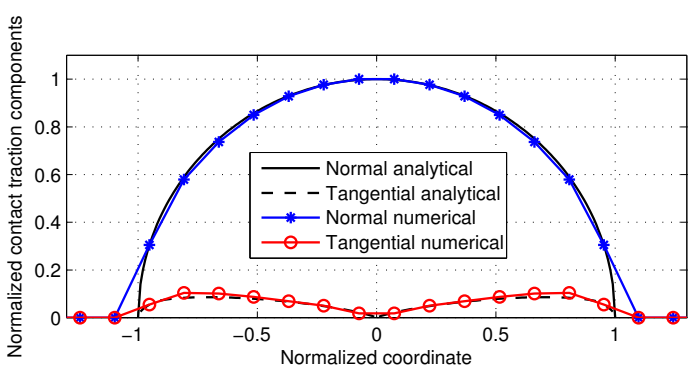

(b)

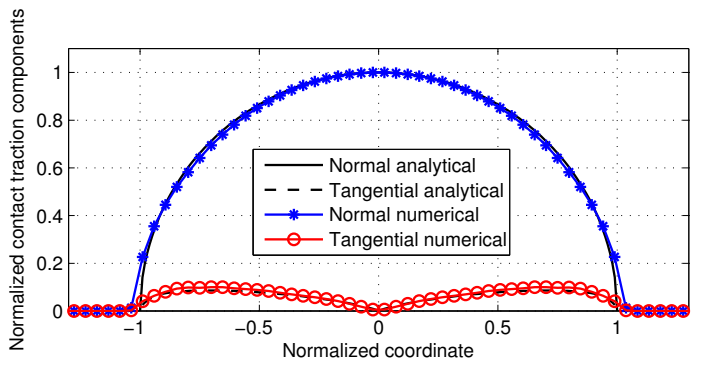

(d)

Figure 19: Example C1: Analytical and numerical normalized contact traction components for meshes with $50 \times 20($ a), $100 \times 30$ (b), $150 \times 40$ (c), $250 \times 60$ (d) control points with discretization order 2. Results obtained with BC. 


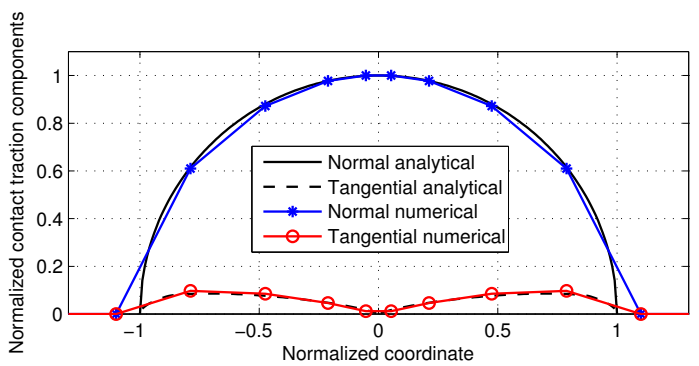

(a)

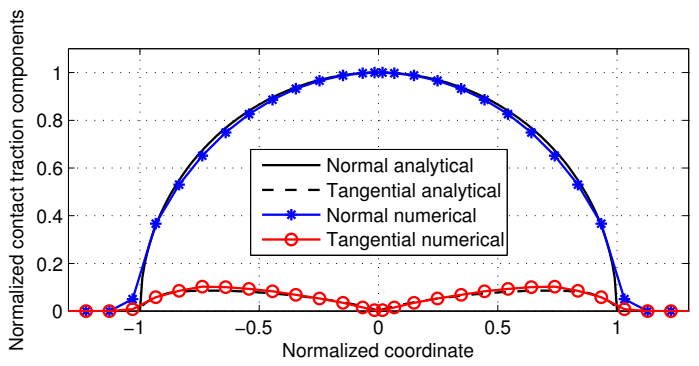

(c)

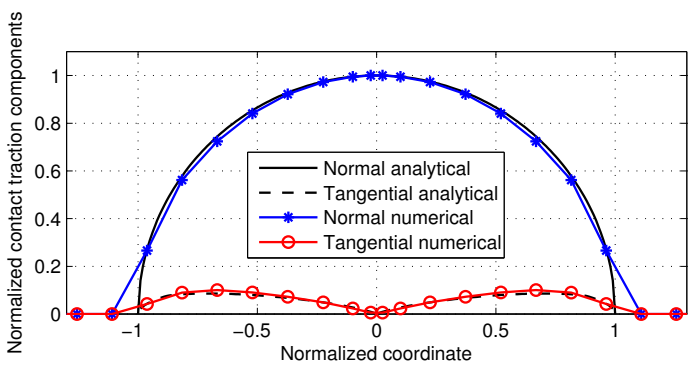

(b)

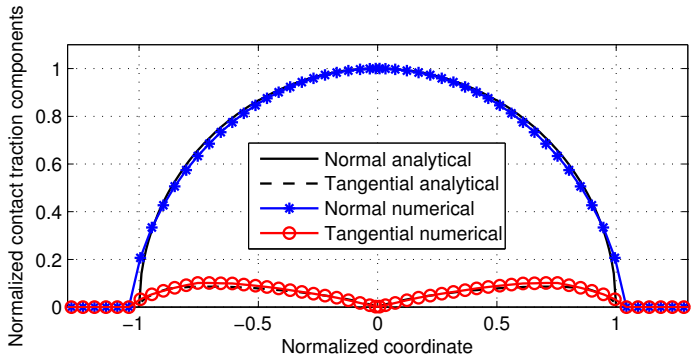

(d)

Figure 20: Example C1: Analytical and numerical normalized contact traction components for meshes with $50 \times 20($ a), $100 \times 30$ (b), $150 \times 40$ (c), $250 \times 60$ (d) control points with discretization order 3. Results obtained with BC. 


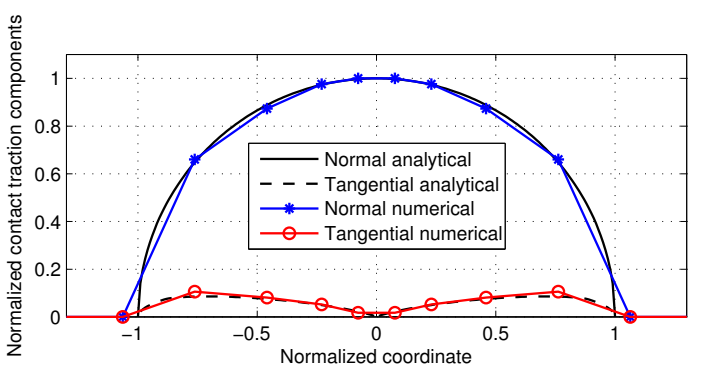

(a)

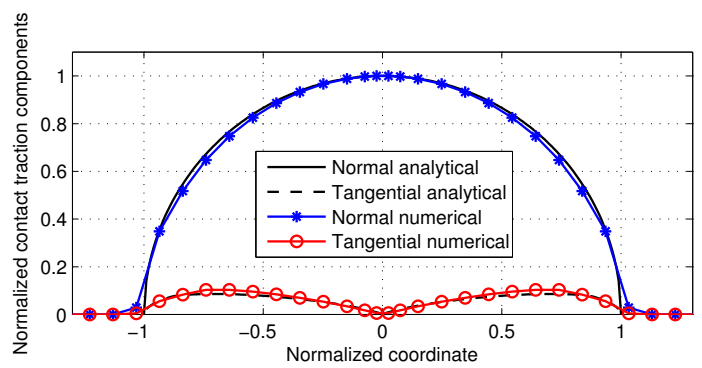

(c)

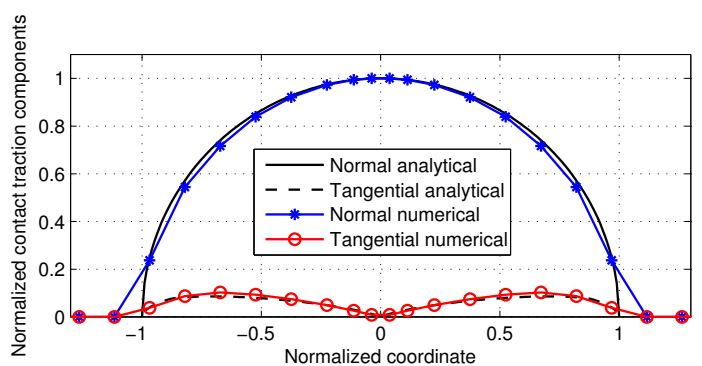

(b)

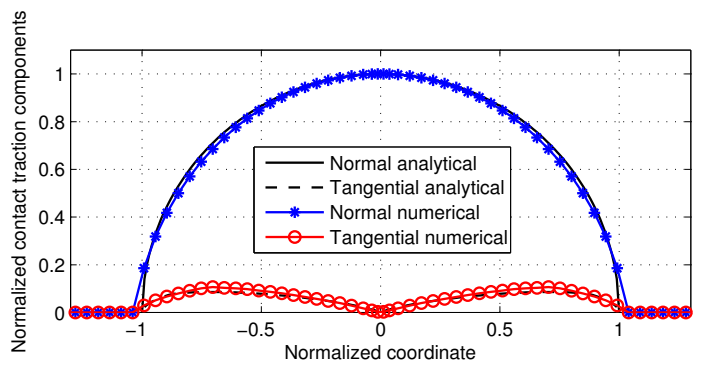

(d)

Figure 21: Example C1: Analytical and numerical normalized contact traction components for meshes with $50 \times 20($ a), $100 \times 30$ (b), $150 \times 40$ (c), $250 \times 60$ (d) control points with discretization order 4. Results obtained with BC. 


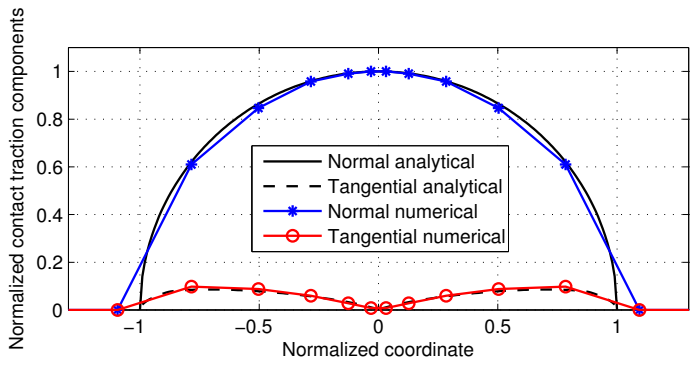

(a)

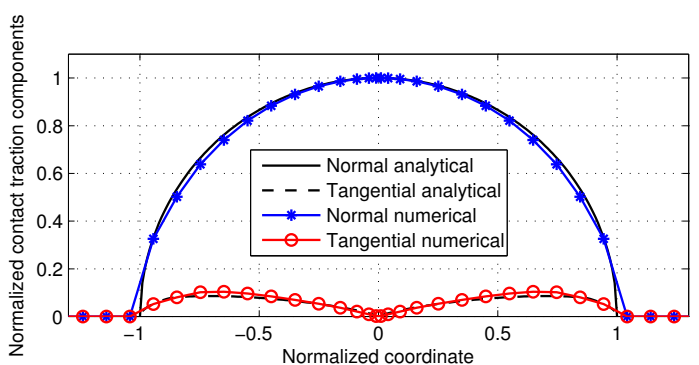

(c)

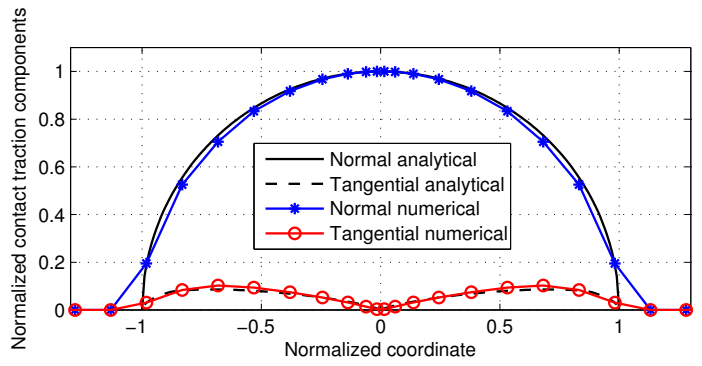

(b)

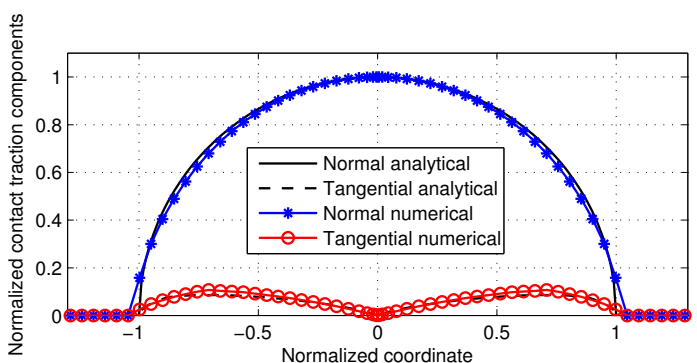

(d)

Figure 22: Example C1: Analytical and numerical normalized contact traction components for meshes with $50 \times 20($ a), $100 \times 30$ (b), $150 \times 40$ (c), $250 \times 60$ (d) control points with discretization order 5. Results obtained with BC.

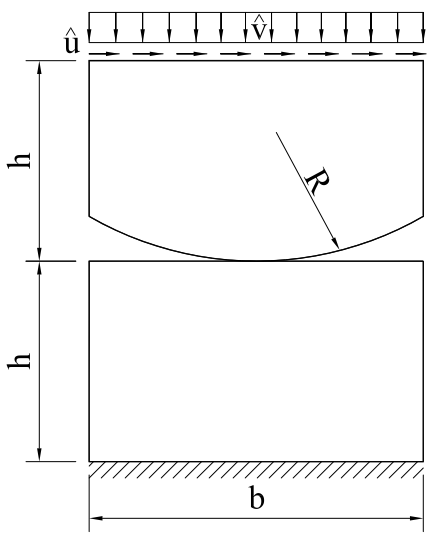

(a)

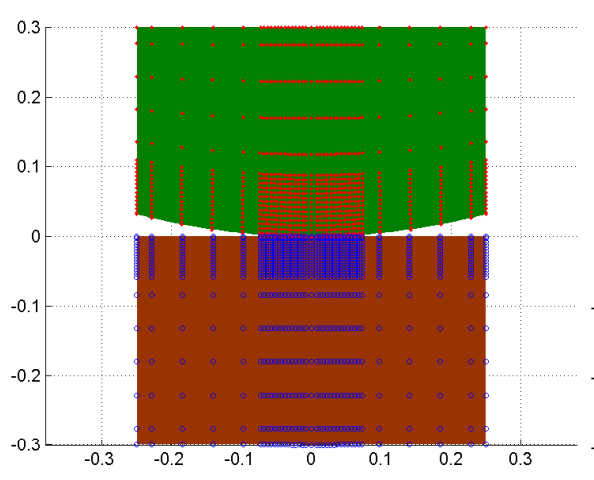

(b)

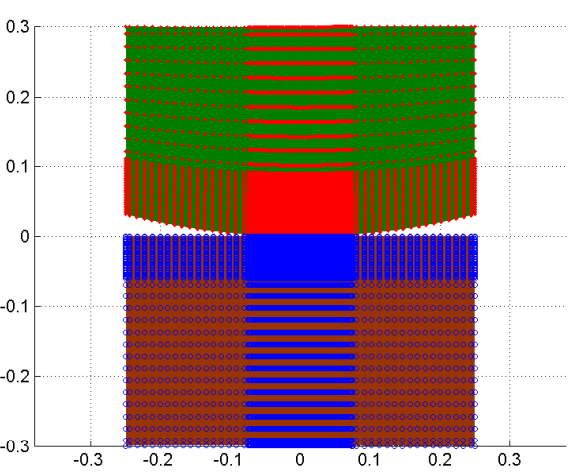

(c)

Figure 23: Example C2: (a) Setup. Collocation points for the coarsest (b) and finest (c) meshes. 


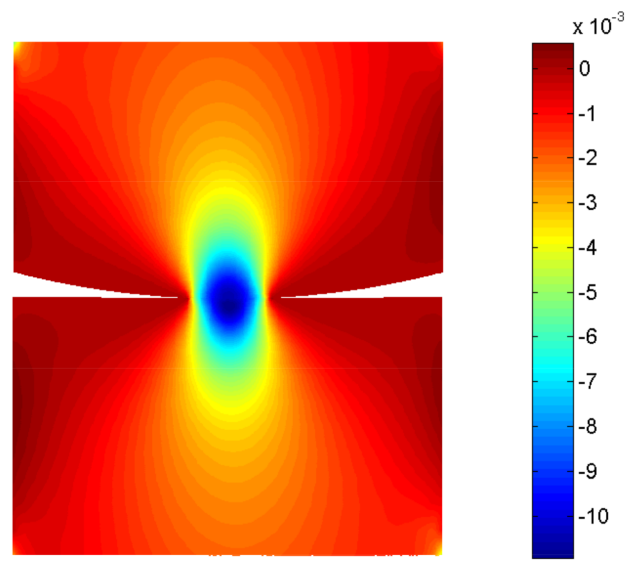

Figure 24: Example C2: Contour plot of $\sigma_{y y}$. Results obtained with BC.

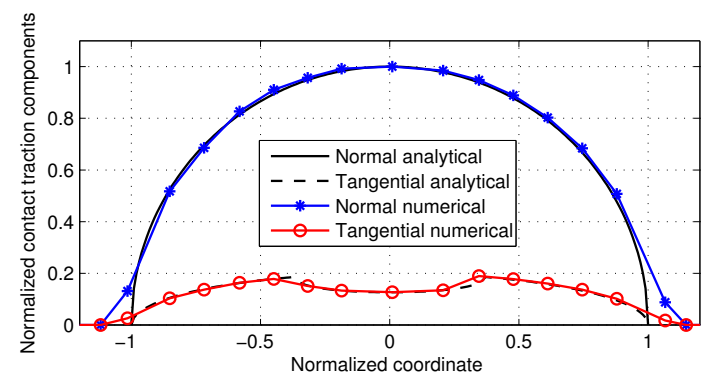

(a)

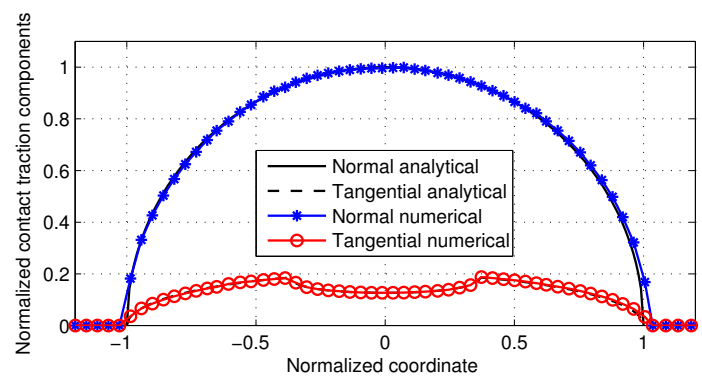

(c)

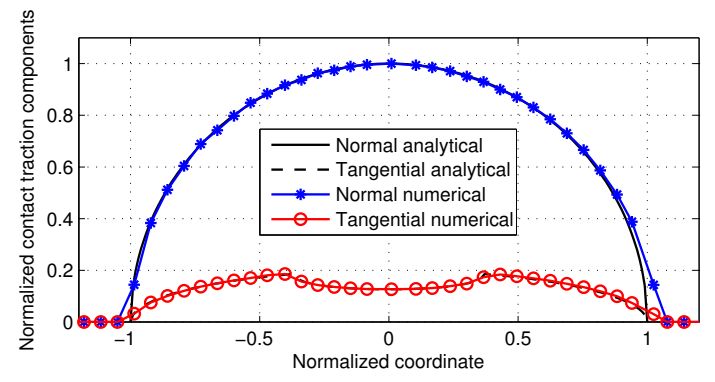

(b)

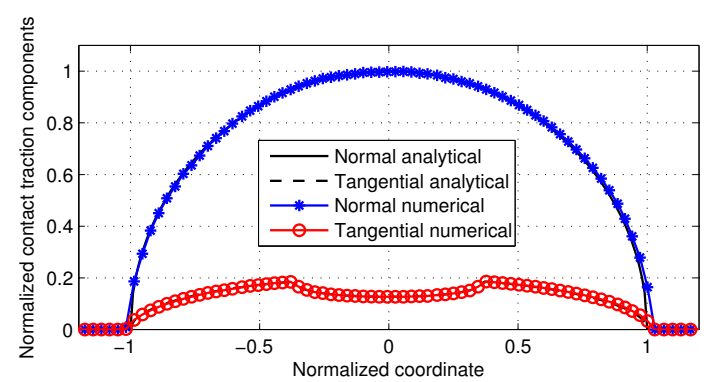

(d)

Figure 25: Example C2: Analytical and numerical normalized contact traction components for meshes with $40 \times 20$ (a), $80 \times 30$ (b), $120 \times 40$ (c), $160 \times 50$ (d) control points with discretization order 2. Results obtained with BC. 


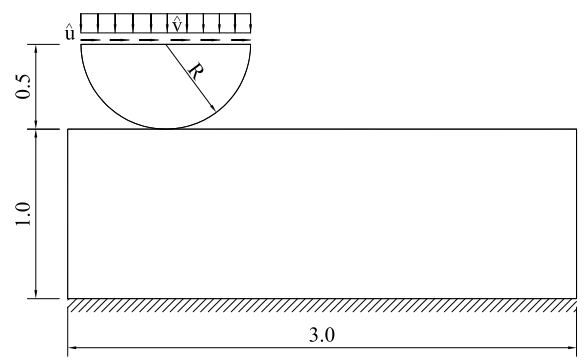

(a)

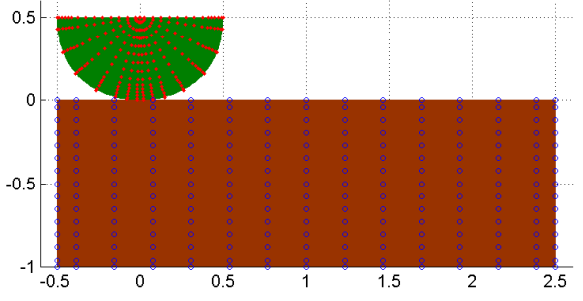

(b)

Figure 26: Example C3: (a) Setup. (b) Collocation points.
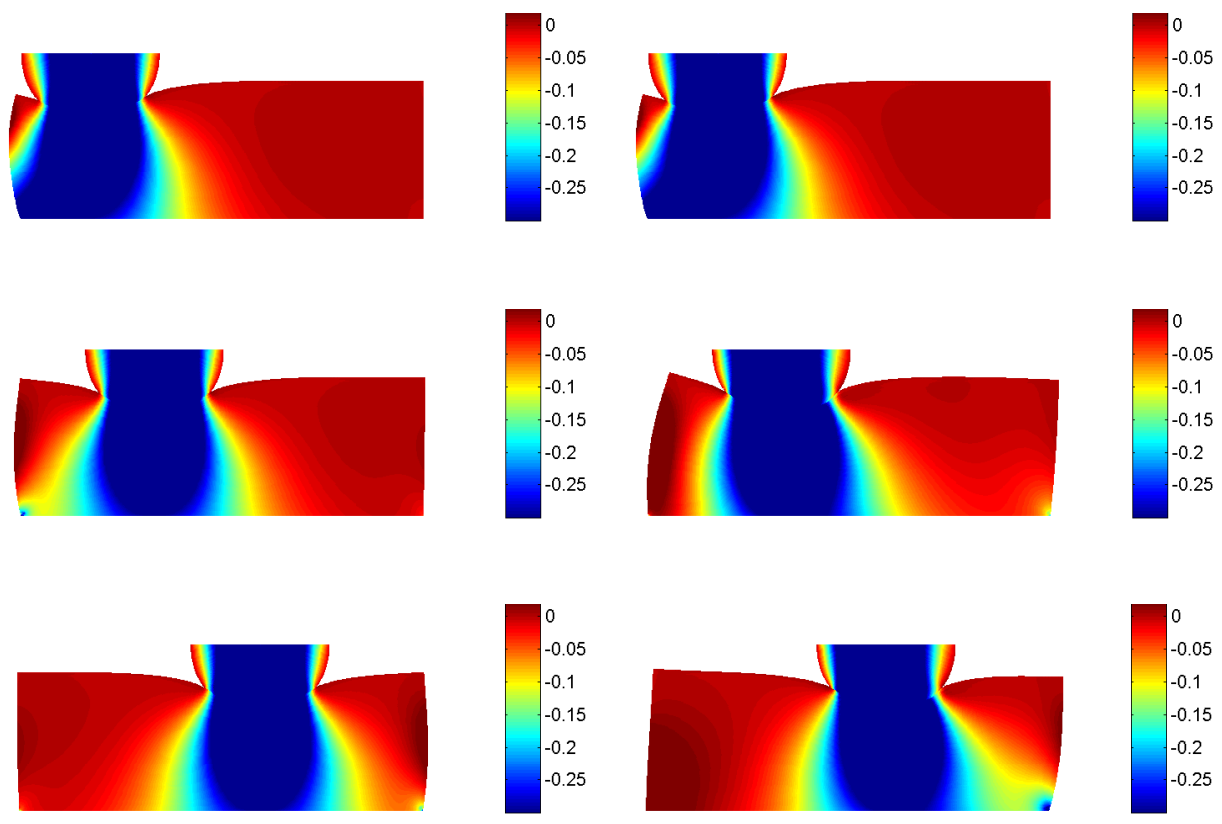

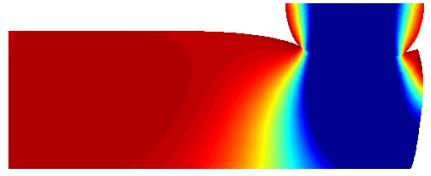

(a)
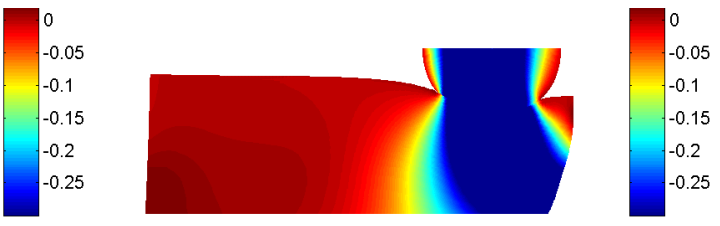

(b)

Figure 27: Example C3: Contour plots of $\sigma_{y y}$ on the deformed configuration at $t=20, t=50, t=100$ and $t=150$, from top to bottom, (a) without friction and (b) with friction $(\mu=0.3)$. Results obtained with BC. 


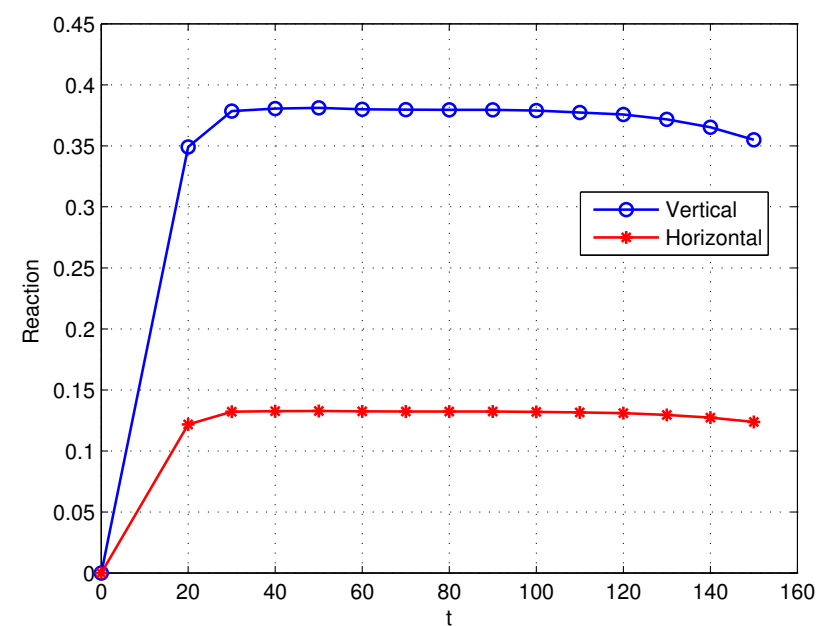

Figure 28: Example C3: Reaction force history. Results obtained with BC.

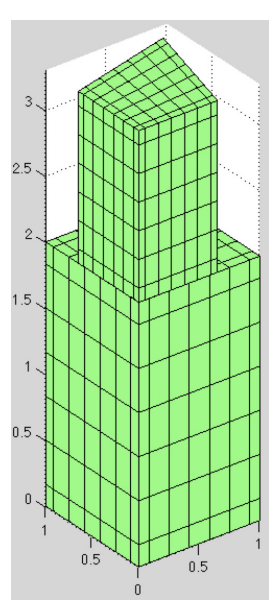

(a)

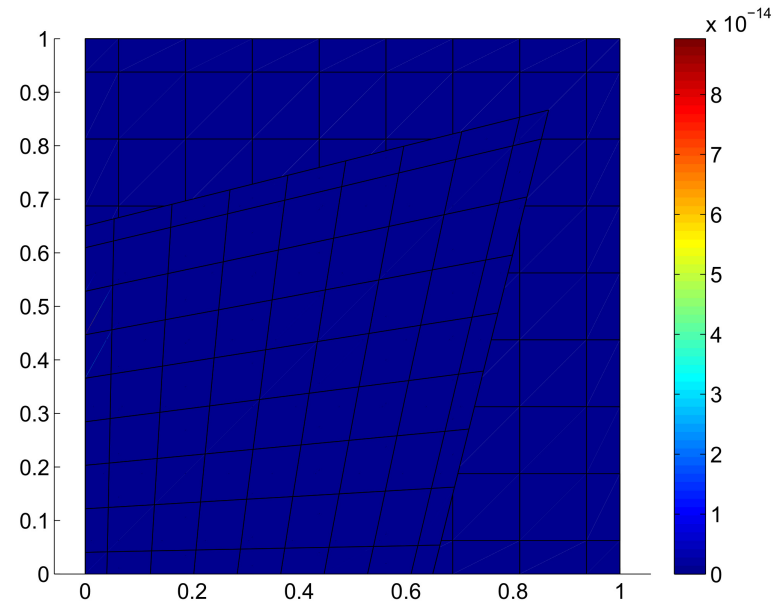

(b)

Figure 29: Example C4: Setup (a). Stress error (b). Results obtained with BC. 
Appendix A. Spatial convergence for enhanced collocation with $C^{*}=15$

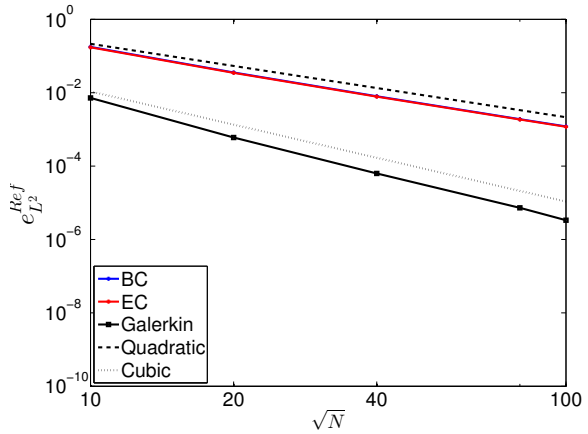

(a)

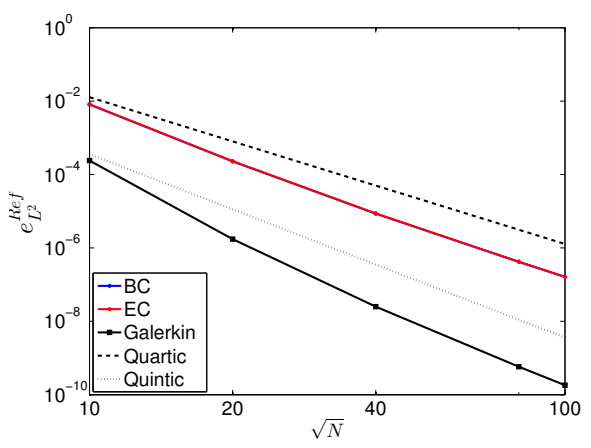

(c)

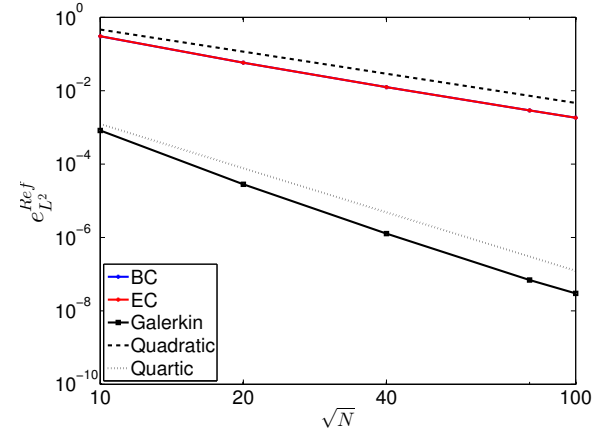

(b)

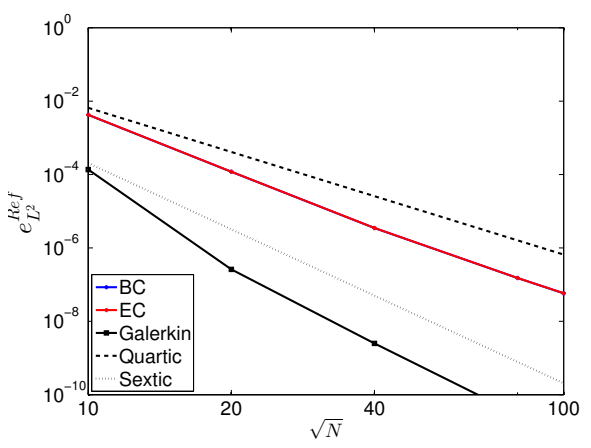

(d)

Figure A.30: Example E1: Spatial convergence (EC with $\left.C^{*}=15\right)$. No-split Neo-Hookean model with discretization order 2 (a), 3 (b), 4 (c), 5 (d). Uniform meshes. 


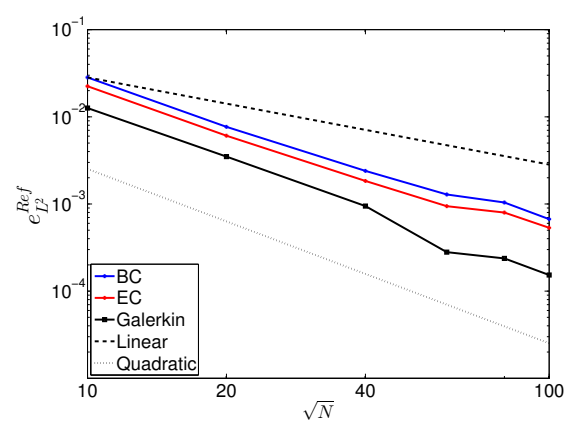

(a)

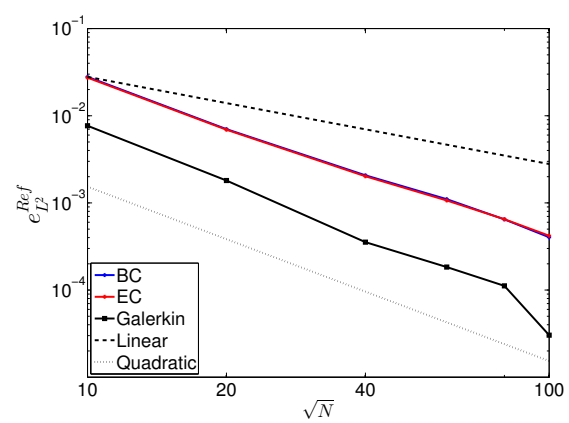

(d)

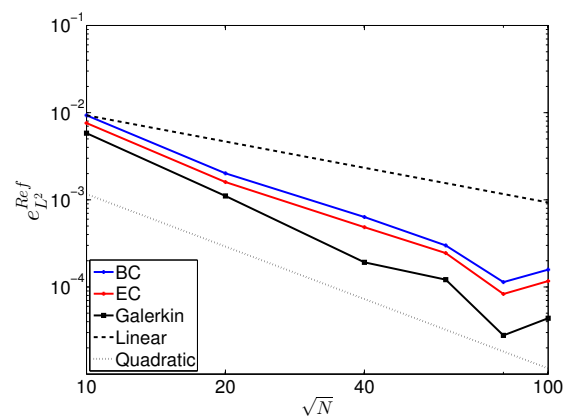

(g)

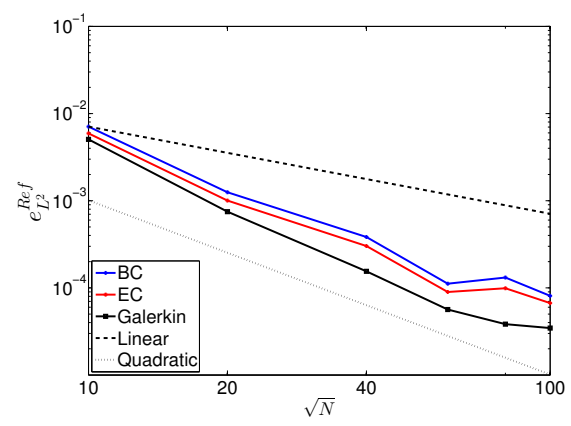

(j)

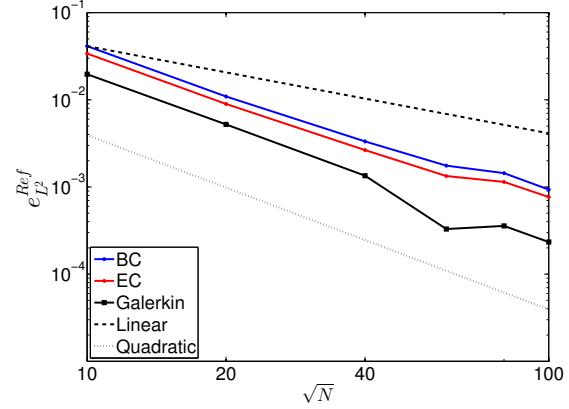

(b)

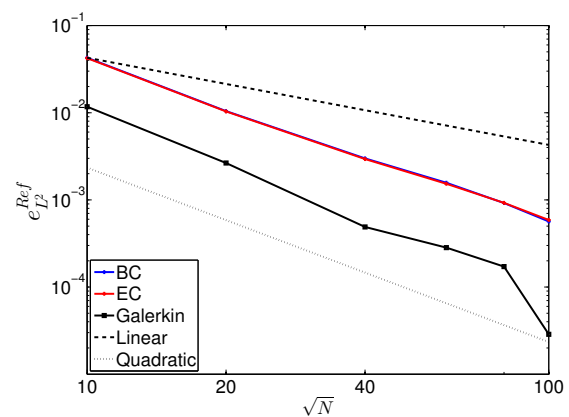

(e)

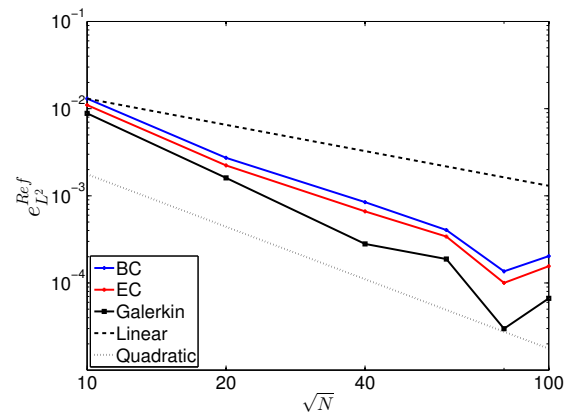

(h)

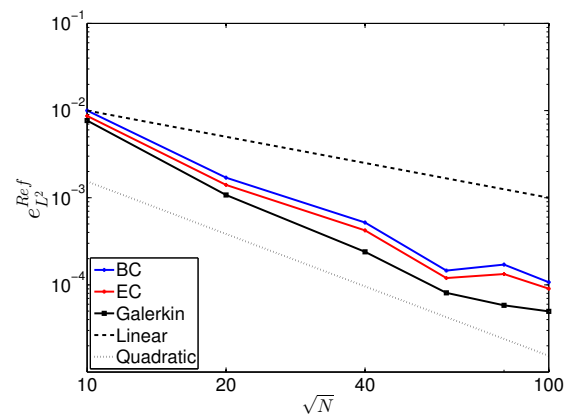

$(\mathrm{k})$

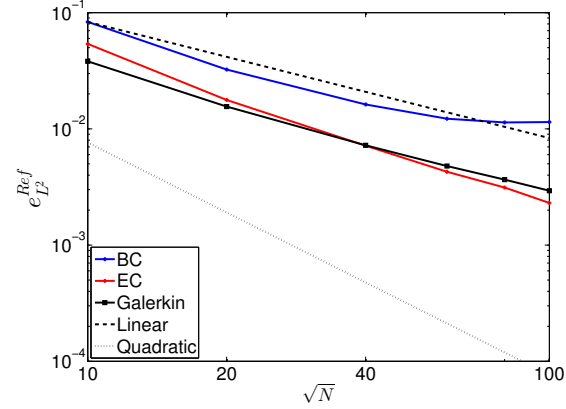

(c)

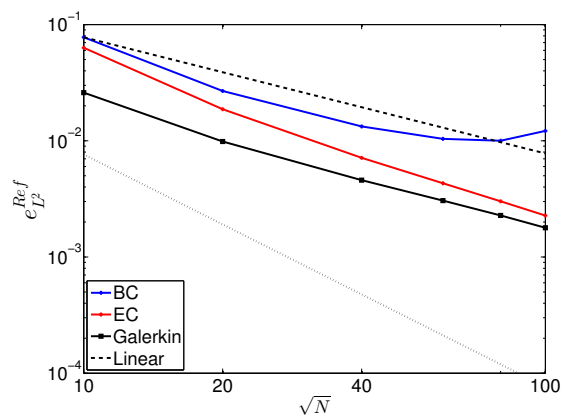

(f)

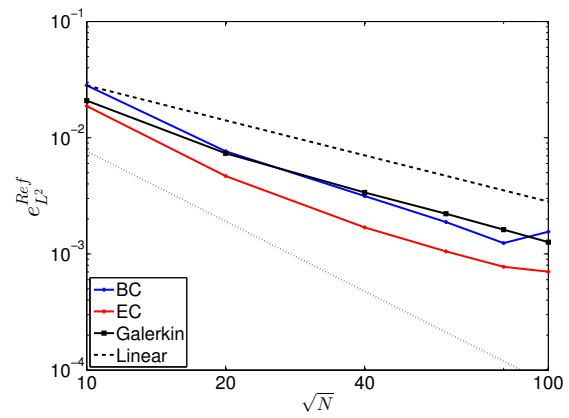

(i)

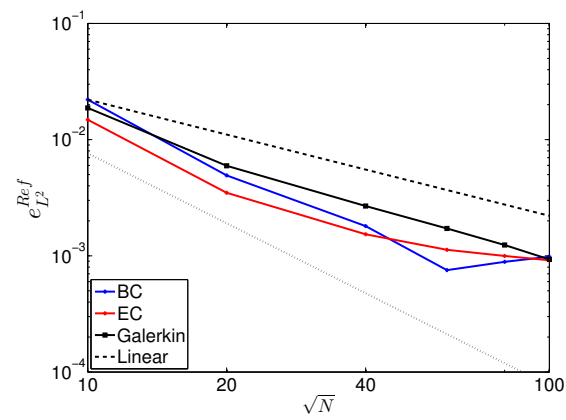

(l)

Figure A.31: Example E2: Spatial convergence (EC with $\left.C^{*}=15\right)$ for $(\mathrm{a}, \mathrm{d}, \mathrm{g}, \mathrm{j}) \hat{v}=0.25$, $(\mathrm{b}, \mathrm{e}, \mathrm{h}, \mathrm{k}) \hat{v}=0.50$, and $(\mathrm{c}, \mathrm{f}, \mathrm{i}, \mathrm{l})$ $\hat{v}=-0.20$ with discretization order $2(\mathrm{a}, \mathrm{b}, \mathrm{c}), 3(\mathrm{~d}, \mathrm{e}, \mathrm{f}), 4(\mathrm{~g}, \mathrm{~h}, \mathrm{i}), 5(\mathrm{j}, \mathrm{k}, \mathrm{l})$. No-split Neo-Hookean model and uniform meshes. 


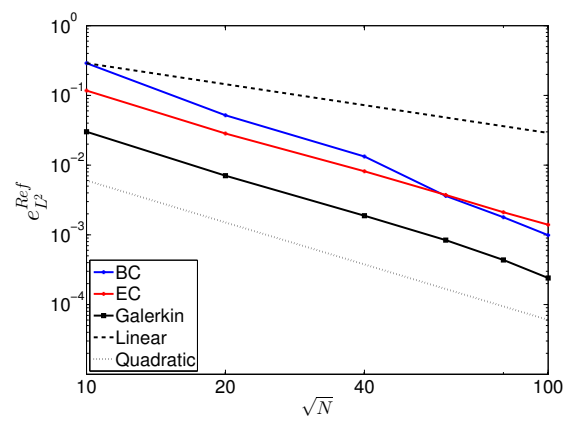

(a)

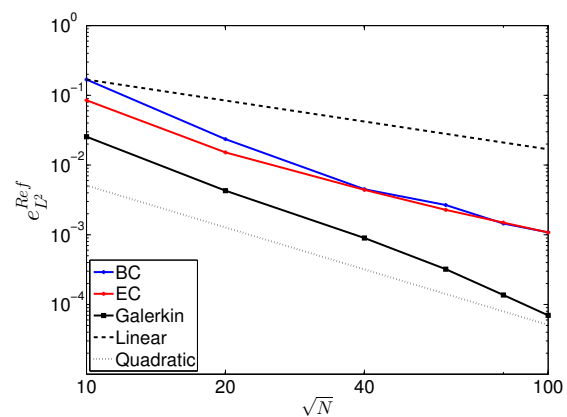

(d)

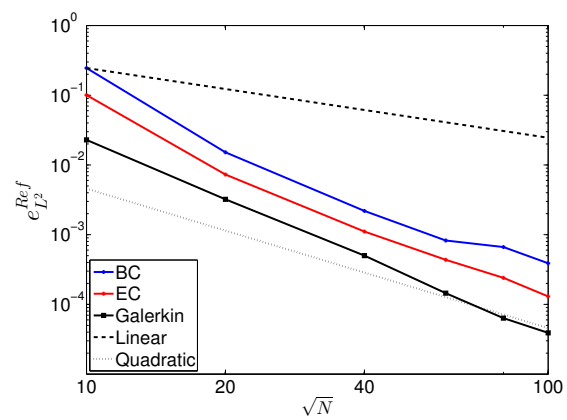

(g)

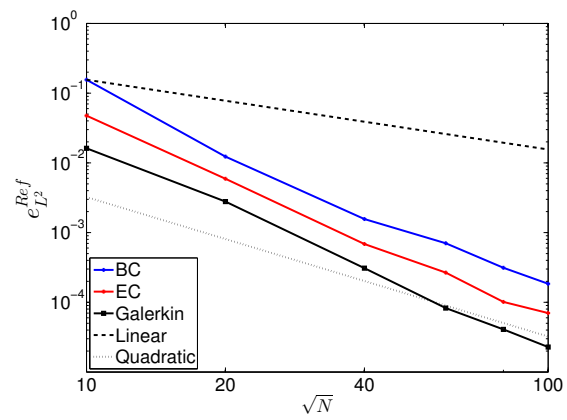

(j)

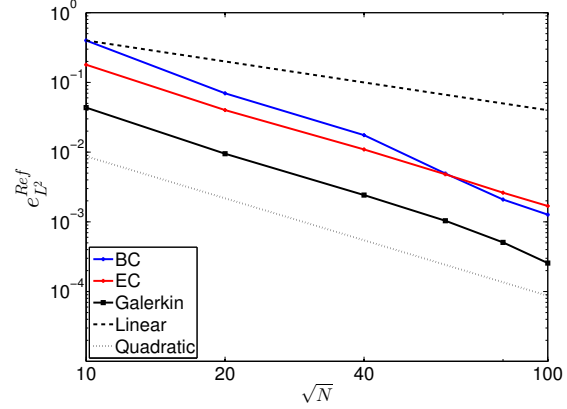

(b)

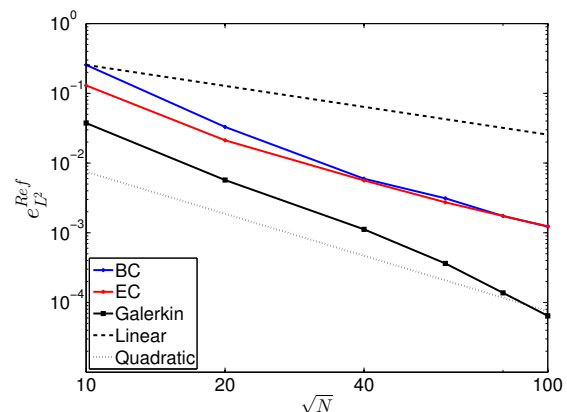

(e)

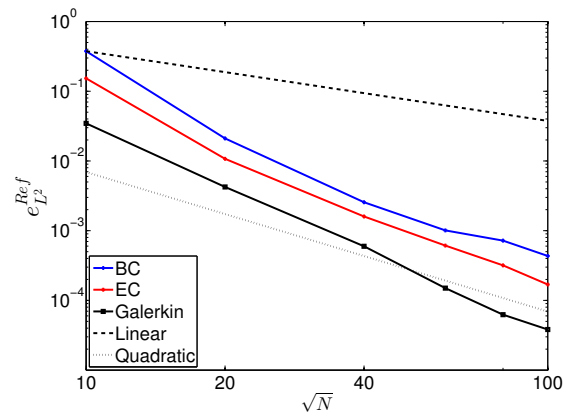

(h)

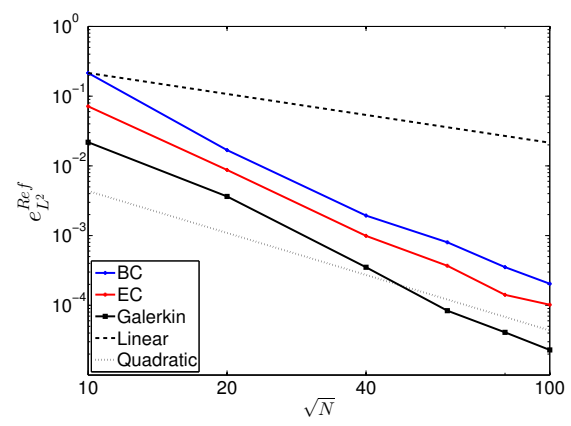

(k)

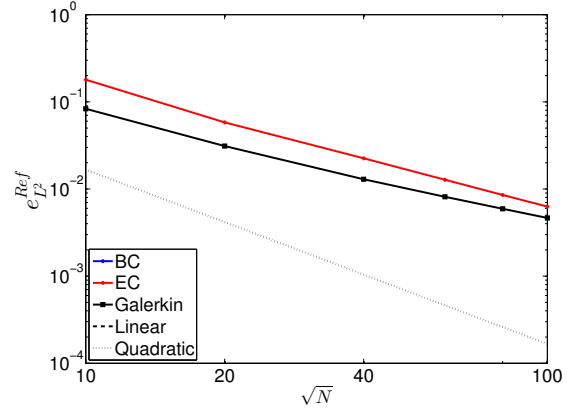

(c)

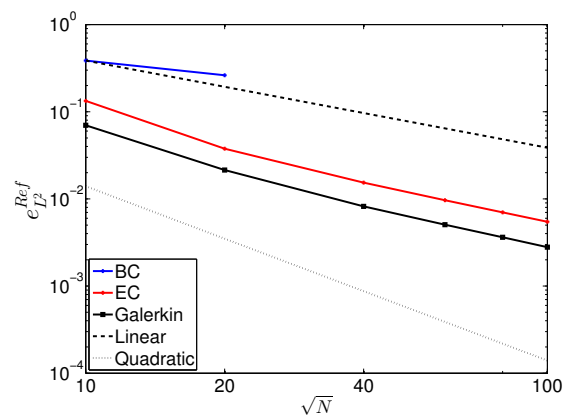

(f)

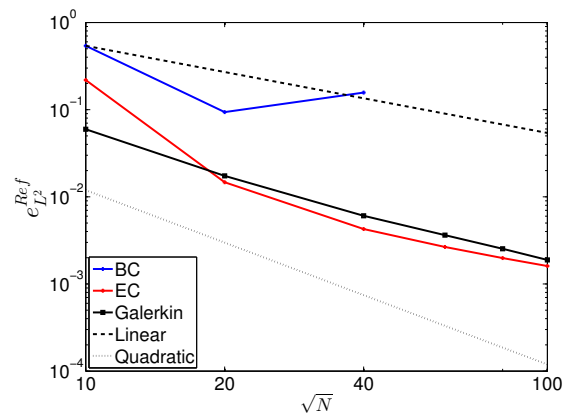

(i)

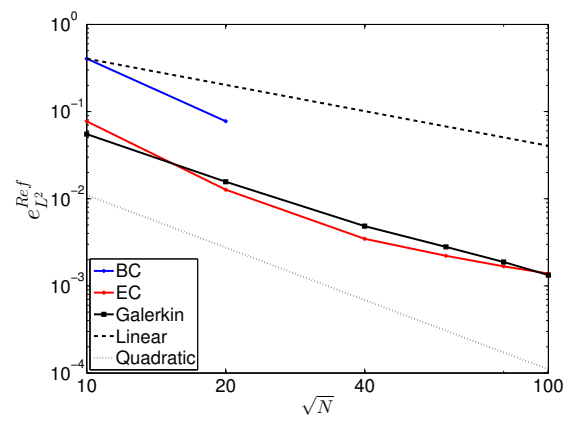

(l)

Figure A.32: Example E2: Spatial convergence (EC with $\left.C^{*}=15\right)$ for $(\mathrm{a}, \mathrm{d}, \mathrm{g}, \mathrm{j}) \hat{v}=0.25$, $(\mathrm{b}, \mathrm{e}, \mathrm{h}, \mathrm{k}) \hat{v}=0.50$, and $(\mathrm{c}, \mathrm{f}, \mathrm{i}, \mathrm{l})$ $\hat{v}=-0.20$ with discretization order $2(\mathrm{a}, \mathrm{b}, \mathrm{c}), 3(\mathrm{~d}, \mathrm{e}, \mathrm{f}), 4(\mathrm{~g}, \mathrm{~h}, \mathrm{i}), 5(\mathrm{j}, \mathrm{k}, \mathrm{l})$. No-split Neo-Hookean model and non-uniform meshes (1:4 aspect ratio). 


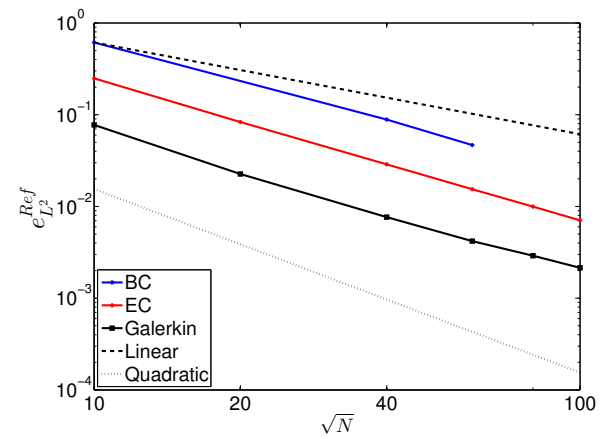

(a)

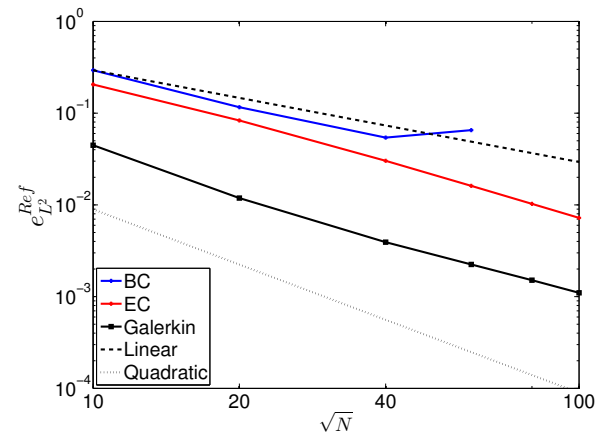

(c)

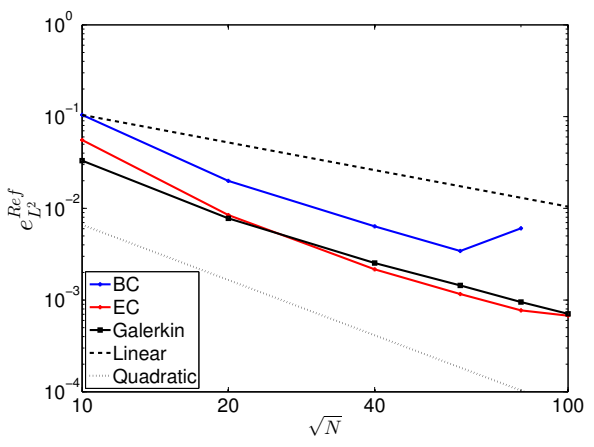

(e)

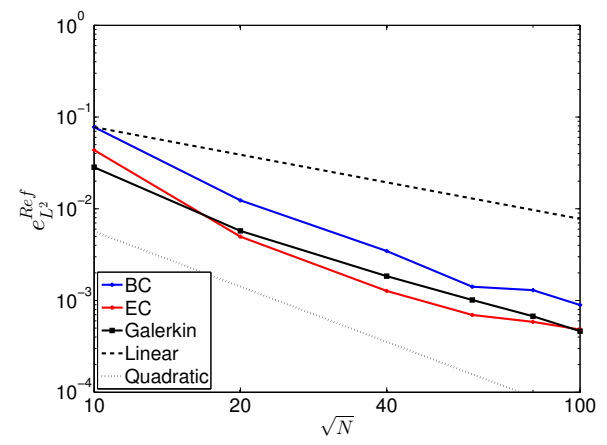

(g)

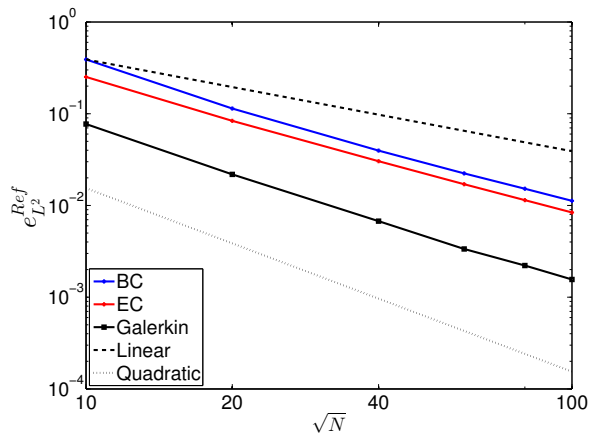

(b)

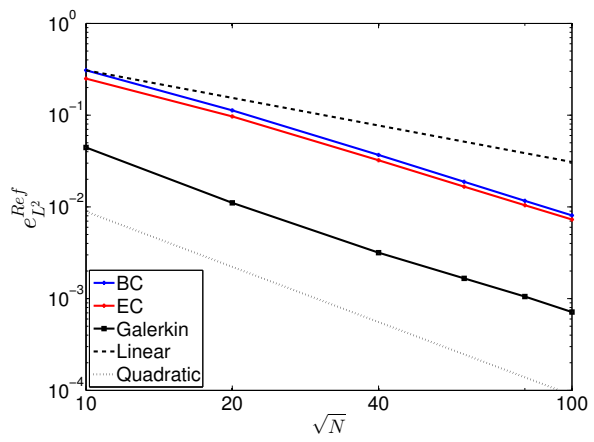

(d)

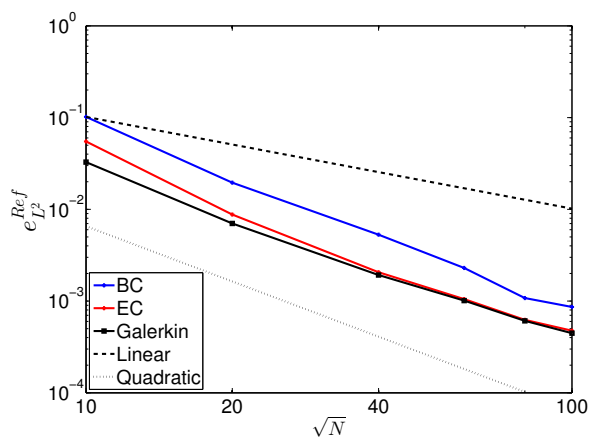

(f)

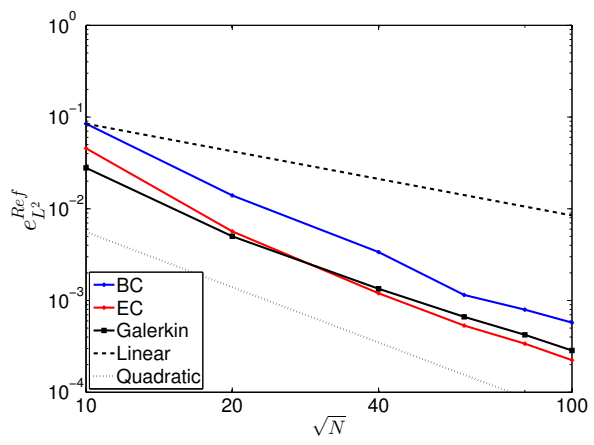

(h)

Figure A.33: Example E3: Spatial convergence (EC with $\left.C^{*}=15\right)$. (a,c,e,g) No-split Neo-Hookean model, (b,d,f,h) split Neo-Hookean model with discretization order $2(\mathrm{a}, \mathrm{b}), 3(\mathrm{c}, \mathrm{d}), 4(\mathrm{e}, \mathrm{f}), 5(\mathrm{~g}, \mathrm{~h})$. Uniform meshes. 


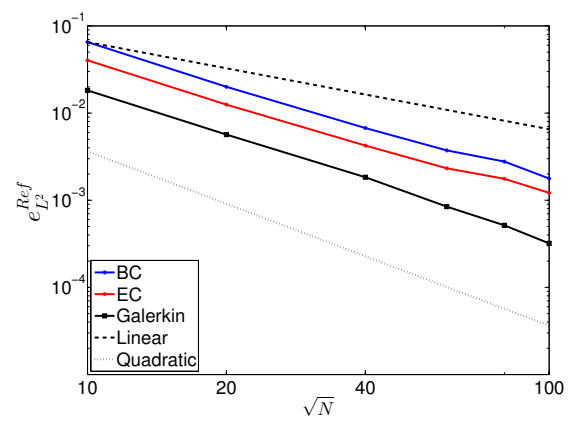

(a)

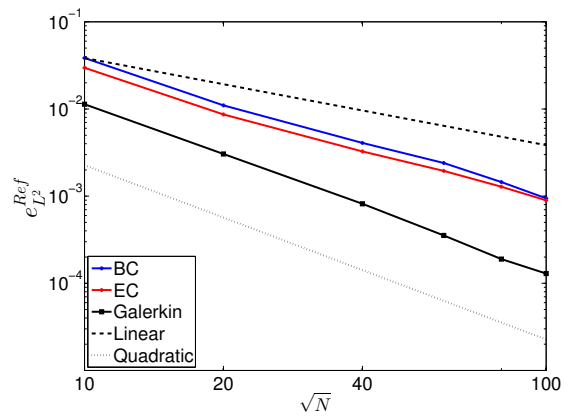

(d)

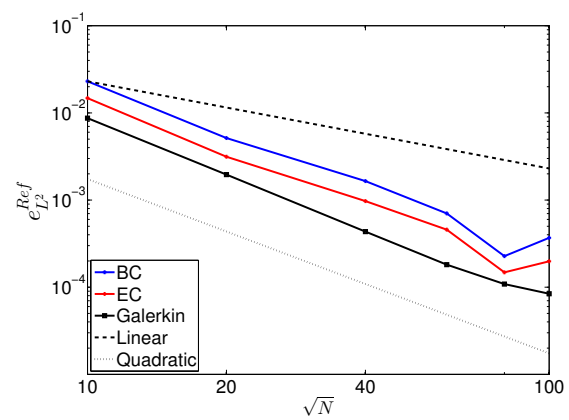

(g)

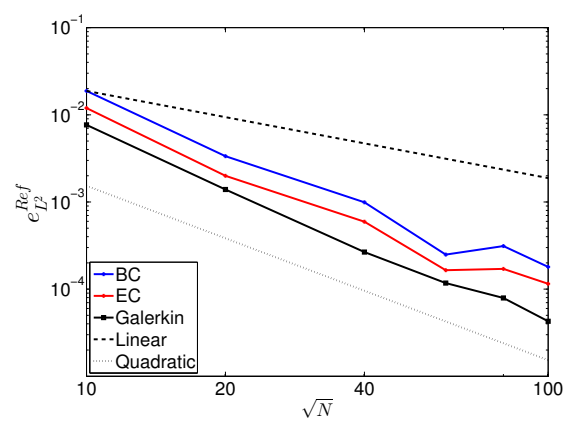

(j)

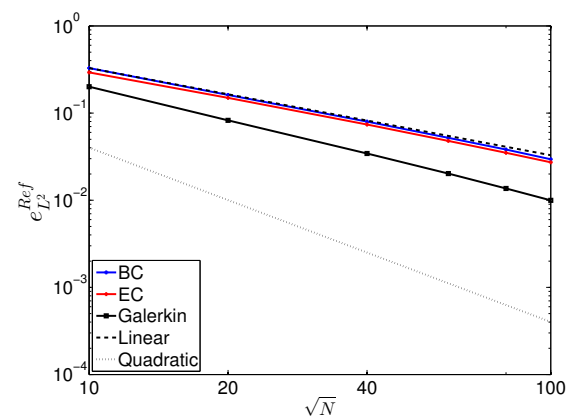

(b)

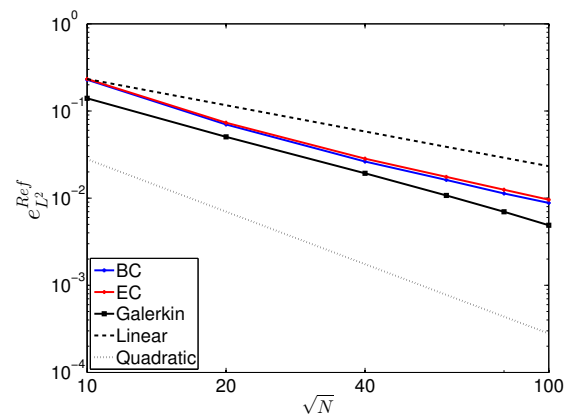

(e)

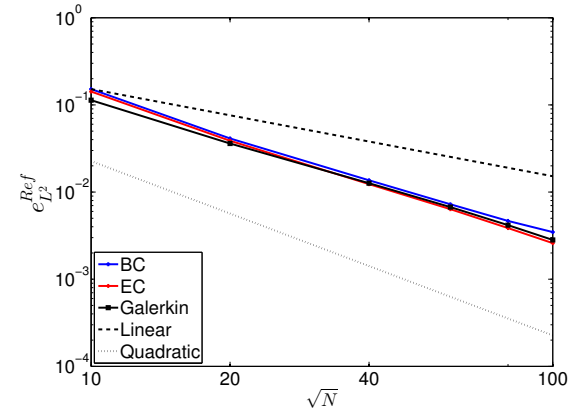

(h)

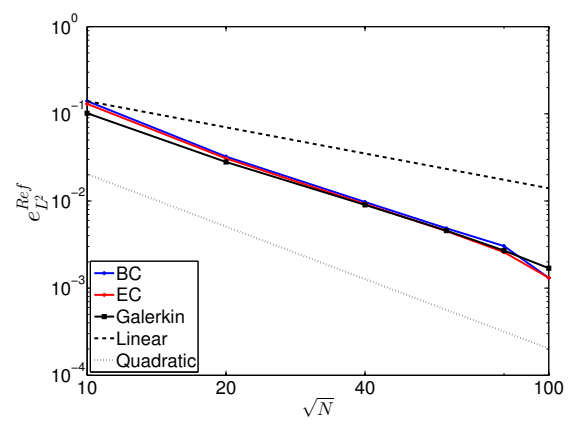

(k)

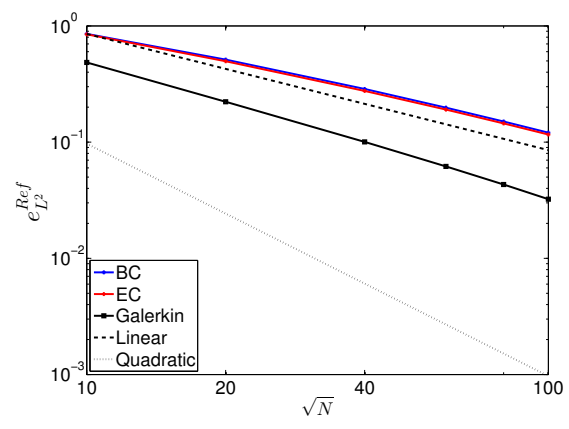

(c)

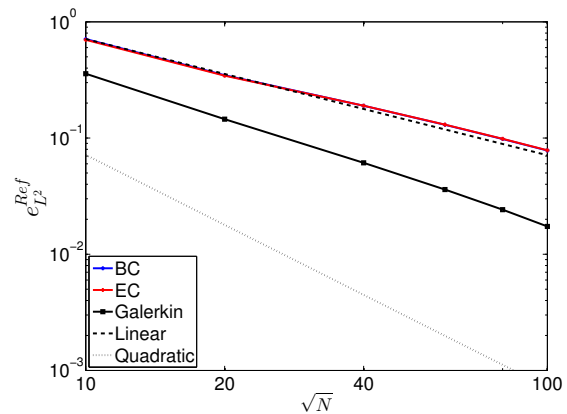

(f)

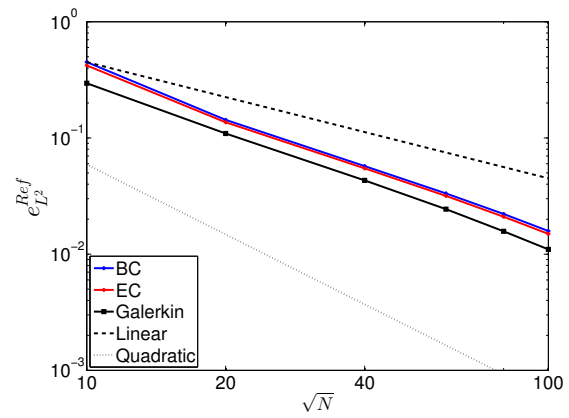

(i)

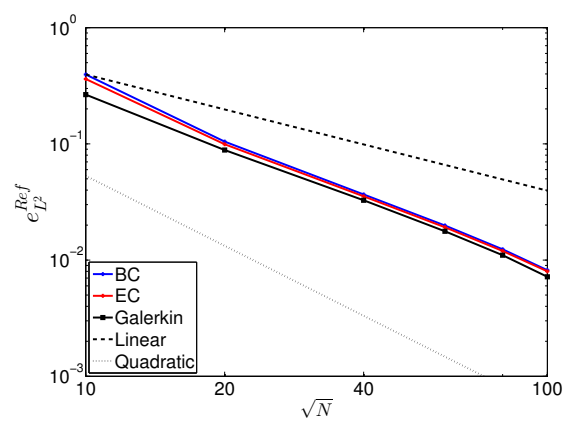

(l)

Figure A.34: Example E4: Spatial convergence (EC with $\left.C^{*}=15\right)$. (a,d,g,j) Square, (b,e,h,k) barrel-shape $1\left(136^{\circ}\right),(\mathrm{c}, \mathrm{f}, \mathrm{i}, \mathrm{l})$ barrel-shape $2\left(154^{\circ}\right)$ with discretization order $2(\mathrm{a}, \mathrm{b}, \mathrm{c}), 3(\mathrm{~d}, \mathrm{e}, \mathrm{f}), 4(\mathrm{~g}, \mathrm{~h}, \mathrm{i}), 5(\mathrm{j}, \mathrm{k}, \mathrm{l})$. Small deformation elasticity and uniform meshes. 


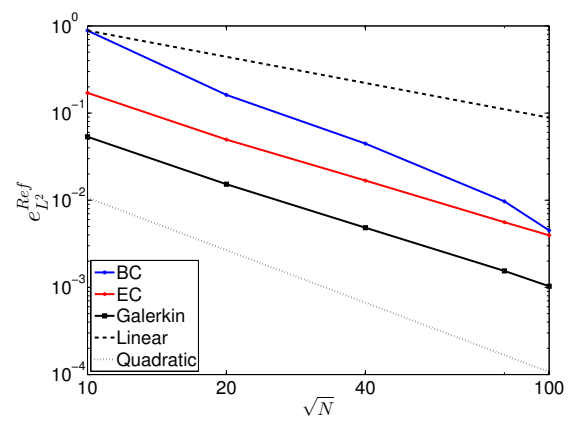

(a)

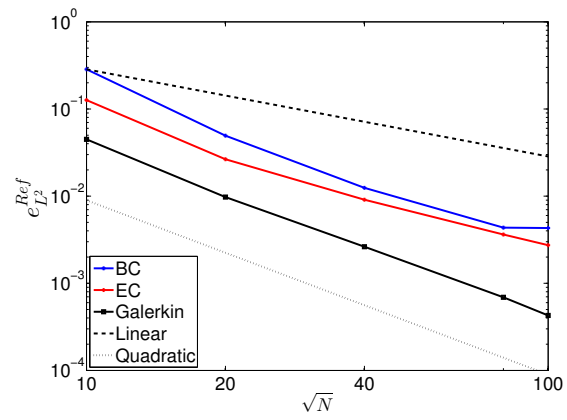

(d)

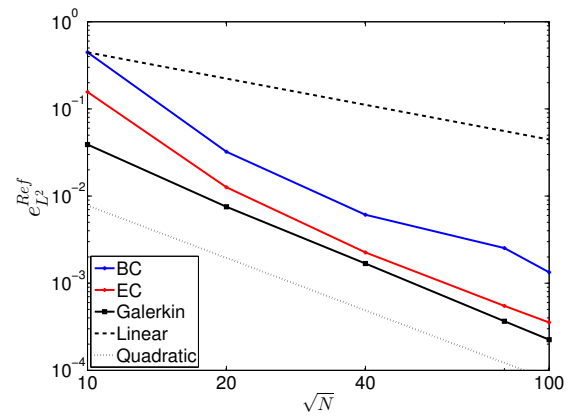

(g)

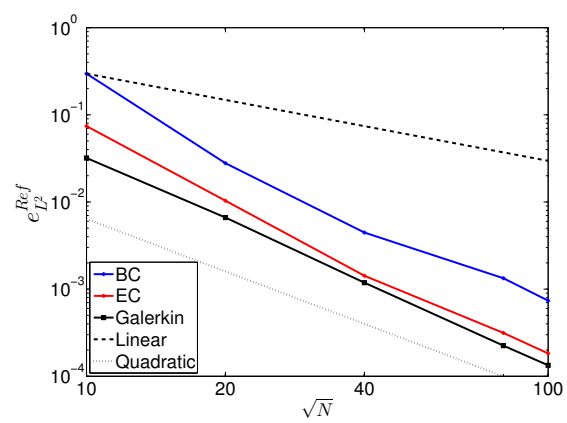

(j)

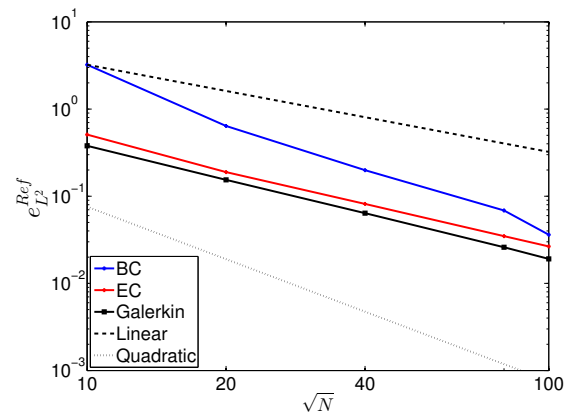

(b)

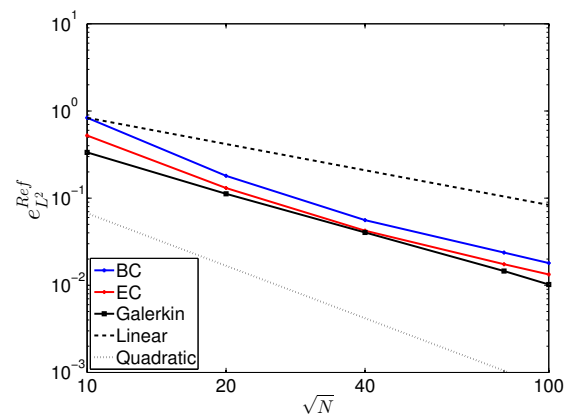

(e)

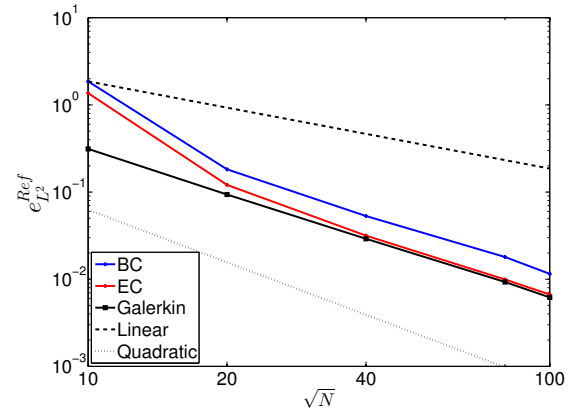

(h)

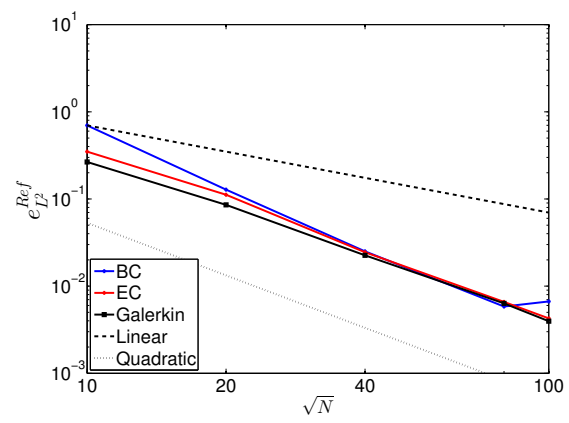

(k)

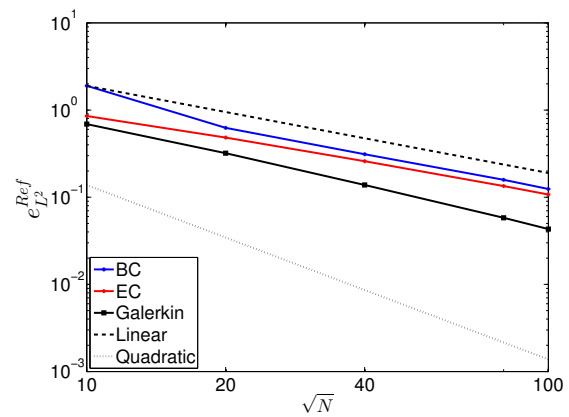

(c)

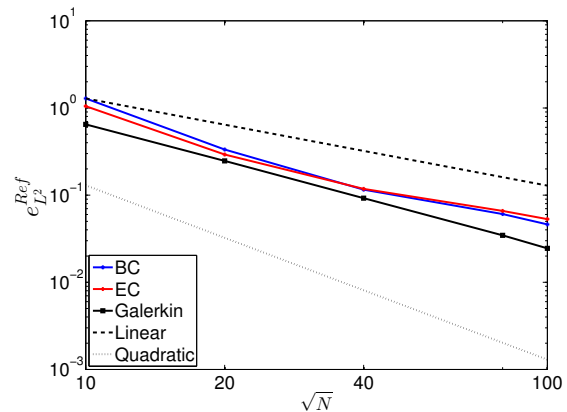

(f)

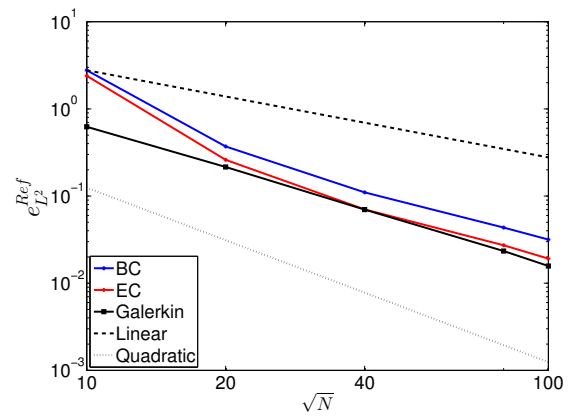

(i)

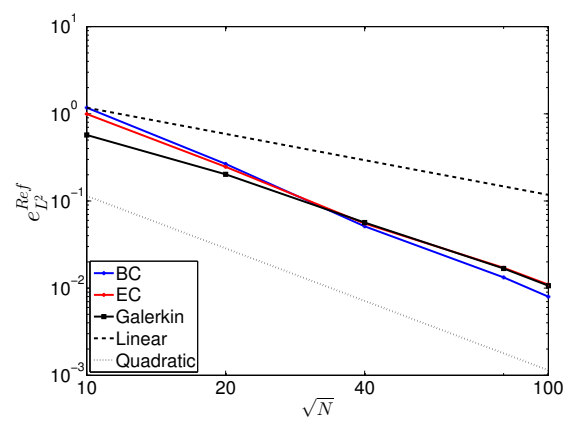

(l)

Figure A.35: Example E4: Spatial convergence (EC with $\left.C^{*}=15\right)$. (a,d,g,j) Square, (b,e,h,k) barrel-shape $1\left(136^{\circ}\right),(\mathrm{c}, \mathrm{f}, \mathrm{i}, \mathrm{l})$ barrel-shape $2\left(154^{\circ}\right)$ with discretization order $2(\mathrm{a}, \mathrm{b}, \mathrm{c}), 3(\mathrm{~d}, \mathrm{e}, \mathrm{f}), 4(\mathrm{~g}, \mathrm{~h}, \mathrm{i}), 5(\mathrm{j}, \mathrm{k}, \mathrm{l})$. Small deformation elasticity and non-uniform meshes (1:4 aspect ratio). 


\section{Appendix B. Consistent linearization of the contact term}

Eqs. (69) and (70), respectively for the BC and EC collocation approaches, contain in different forms the same boundary residual term

$$
\mathbf{R}_{b o u}=\boldsymbol{\sigma n}-\mathbf{t}
$$

(Appendix B.1)

where $\mathbf{t}$ is given by eq. (59). For a more concise notation, the superscript $h$ indicating discretization is here omitted. Linearization of this residual gives

$$
\Delta \mathbf{R}_{b o u}=\Delta(\boldsymbol{\sigma} \mathbf{n})-\Delta \mathbf{t}
$$

with $\Delta$ as the symbol for linearized increment. As follows, we focus on the computation of $\Delta \mathbf{t}$ which is related to the contact formulation. From eq. (59) follows

$$
\Delta \mathbf{t}=\Delta t_{N} \overline{\mathbf{n}}^{(m)}+t_{N} \Delta \overline{\mathbf{n}}^{(m)}+\Delta t_{T}^{1} \overline{\boldsymbol{\tau}}_{1}^{(m)}+t_{T}^{1} \Delta \overline{\boldsymbol{\tau}}_{1}^{(m)}
$$

(Appendix B.3)

In the latter equation it has been assumed that the master surface is parameterized by the convective coordinate $\xi$, that defines the covariant vector $\boldsymbol{\tau}_{1}^{(m)}=\mathbf{x}_{, \xi}^{(m)}$ and the metric $m_{11}^{(m)}:=\boldsymbol{\tau}_{1}^{(m)} \cdot \boldsymbol{\tau}_{1}^{(m)}$. The curvature follows from $k_{11}^{(m)}=\mathbf{x}_{\xi \xi}^{(m)} \cdot \mathbf{n}^{(m)}$, where $\mathbf{n}^{(m)}$ is the normal unit vector to the master surface. As these quantities are all evaluated at the projection point, the parametric location of this point must also be linearized. It can be shown that $[27,28]$

$$
\begin{gathered}
\Delta \overline{\mathbf{n}}^{(m)}=-\frac{1}{\bar{m}_{11}^{(m)}}\left(\Delta \overline{\mathbf{x}}_{, \xi}^{(m)} \cdot \overline{\mathbf{n}}^{(m)}+\bar{k}_{11}^{(m)} \Delta \bar{\xi}^{(m)}\right) \overline{\boldsymbol{\tau}}_{1}^{(m)} \\
\Delta \overline{\boldsymbol{\tau}}_{1}^{(m)}=\Delta \overline{\mathbf{x}}_{, \xi}^{(m)}+\overline{\mathbf{x}}_{, \xi \xi}^{(m)} \Delta \bar{\xi}^{(m)}
\end{gathered}
$$

and

$$
\Delta \bar{\xi}^{(m)}=\frac{1}{\bar{A}_{11}^{(m)}}\left[\left(\Delta \mathbf{x}^{(s)}-\Delta \overline{\mathbf{x}}^{(m)}\right) \cdot \overline{\boldsymbol{\tau}}_{1}^{(m)}+g_{N} \overline{\mathbf{n}}^{(m)} \cdot \Delta \overline{\mathbf{x}}_{, \xi}^{(m)}\right]
$$

with

$$
\bar{A}_{11}^{(m)}=\bar{m}_{11}^{(m)}-g_{N} \bar{k}_{11}^{(m)}
$$

From eq. (65), in the active contact case it is

$$
\Delta t_{N}=\varepsilon_{N} \Delta g_{N}
$$

with

$$
\Delta g_{N}=\left(\Delta \mathbf{x}^{(s)}-\Delta \overline{\mathbf{x}}^{(m)}\right) \cdot \overline{\mathbf{n}}^{(m)}
$$

Linearization of the tangential contravariant traction component in the stick case follows from eqs. (68) and (67) as

$$
\Delta t_{T}^{1}=\Delta t_{T}^{1 \mathrm{tr}}=\varepsilon_{T} \Delta \bar{\xi}^{(m)}
$$

whereas in the slip case it is

$$
\Delta t_{T}^{1}=-\mu \Delta t_{N} \frac{t_{T}^{1 \mathrm{tr}}}{\left\|\mathbf{t}_{T}^{\text {tr }}\right\|}-\mu t_{N} \frac{\Delta t_{T}^{1 \mathrm{tr}}}{\left\|\mathbf{t}_{T}^{\mathrm{tr}}\right\|}+\mu t_{N} t_{T}^{1 \mathrm{tr}} \frac{\Delta\left\|\mathbf{t}_{T}^{\text {tr }}\right\|}{\left\|\mathbf{t}_{T}^{\mathrm{tr}}\right\|^{2}}
$$

where

$$
\Delta\left\|\mathbf{t}_{T}^{t r}\right\|=\frac{\mathbf{t}_{T}^{\mathrm{tr}} \cdot \Delta \mathbf{t}_{T}^{\mathrm{tr}}}{\left\|\mathbf{t}_{T}^{\mathrm{tr}}\right\|}
$$

and

$$
\Delta \mathbf{t}_{T}^{\mathrm{tr}}=\Delta t_{T}^{1 \mathrm{tr}} \overline{\boldsymbol{\tau}}_{1}^{(m)}+t_{T}^{1 \mathrm{tr}} \Delta \overline{\boldsymbol{\tau}}_{1}^{(m)}
$$

